\title{
Asymptotic Expansions for Stationary Distributions of Perturbed Semi-Markov Processes
}

\author{
Dmitrii Silvestrov 11 and Sergei Silvestrov2
}

\begin{abstract}
New algorithms for computing of asymptotic expansions for stationary distributions of nonlinearly perturbed semi-Markov processes are presented. The algorithms are based on special techniques of sequential phase space reduction, which can be applied to processes with asymptotically coupled and uncoupled finite phase spaces.

Keywords: Markov chain; semi-Markov process; nonlinear perturbation; stationary distribution; expected hitting time; Laurent asymptotic expansion

2010 Mathematics Subject Classification: Primary 60J10, 60J27, 60K15, Secondary 65C40.
\end{abstract}

\section{Introduction}

In this paper, we present new algorithms for construction asymptotic expansions for stationary distributions of nonlinearly perturbed semi-Markov processes with a finite phase space.

We consider models, where the phase space of embedded Markov chains for pre-limiting perturbed semi-Markov processes is one class of communicative states, while the phase space for the limiting embedded Markov chain can consist of one or several closed classes of communicative states and, possibly, a class of transient states.

The initial perturbation conditions are formulated in the forms of Taylor asymptotic expansions for transition probabilities of the corresponding embedded Markov chains and Laurent asymptotic expansions for expectations

\footnotetext{
${ }^{1}$ Department of Mathematics, Stockholm University, SE-106 81 Stockholm, Sweden. Email address: silvestrov@math.su.se

${ }^{2}$ Division of Applied Mathematics, School of Education, Culture and Communication, Mälardalen University, SE-721 23 Västerås, Sweden.

Email address: sergei.silvestrov@mdh.se
} 
of sojourn times for perturbed semi-Markov processes. Two forms of these expansions are considered, with remainders given without or with explicit upper bounds.

The algorithms are based on special time-space screening procedures for sequential phase space reduction and algorithms for re-calculation of asymptotic expansions and upper bounds for remainders, which constitute perturbation conditions for the semi-Markov processes with reduced phase spaces.

The final asymptotic expansions for stationary distributions of nonlinearly perturbed semi-Markov processes are given in the form of Taylor asymptotic expansions with remainders given without or with explicit upper bounds.

The model of perturbed Markov chains and semi-Markov processes, in particular, in the most difficult case of so-called singularly perturbed Markov chains and semi-Markov processes with absorption and asymptotically uncoupled phase spaces, attracted attention of researchers in the mid of the 20 th century.

The first works related to asymptotical problems for the above models are Meshalkin (1958), Simon, and Ando (1961), Hanen (1963a, b, c, d), Seneta (1967, 1968a, b), Schweitzer (1968), and Korolyuk (1969).

The methods used for construction of asymptotic expansions for stationary distributions and related functionals such as moments of hitting times can be split in three groups.

The first and the most widely used methods are based on analysis of generalized matrix and operator inverses of resolvent type for transition matrices and operators for singularly perturbed Markov chains and semi-Markov processes. Mainly models with linear, polynomial and analytic perturbations have been objects of studies. We refer here to works by Schweitzer (1968), Turbin (1972), Poliščuk and Turbin (1973), Koroljuk, Brodi and Turbin (1974), Pervozvanskiu and Smirnov (1974), Courtois and Louchard (1976), Korolyuk and Turbin $(1976,1978)$, Courtois (1977), Latouche and Louchard (1978), Kokotović, Phillips and Javid (1980), Korolyuk, Penev and Turbin (1981), Phillips and Kokotović (1981), Delebecque (1983), Abadov (1984), Silvestrov and Abadov (1984, 1991, 1993), Kartashov (1985d, 1988, 1996b), Haviv (1986), Korolyuk (1989), Stewart and Sun (1990), Haviv, Ritov and Rothblum (1992), Haviv and Ritov (1993), Schweitzer and Stewart (1993), Stewart (1998, 2001), Yin and Zhang (1998, 2003, 2005, 2013), Avrachenkov (1999, 2000), Avrachenkov and Lasserre (1999), Korolyuk, V.S. and Korolyuk, V.V. (1999), Yin, G., Zhang, Yang and Yin, K. (2001), Avrachenkov and Haviv (2003, 2004), Craven (2003), Bini, Latouche and Meini (2005), 
Korolyuk and Limnios (2005) and Avrachenkov, Filar and Howlett (2013).

Alternatively, the methods based on regenerative properties of Markov chains and semi-Markov processes, in particular, relations which link stationary probabilities and expectations of return times have been used for getting approximations for expectations of hitting times and stationary distributions in works by Grassman, Taksar and Heyman (1985), Hassin and Haviv (1992) and Hunter (2005). Also, the above mentioned relations and methods based on asymptotic expansions for nonlinearly perturbed regenerative processes developed in works by Silvestrov $(1995,2007,2010)$, Englund and Silvestrov (1997), Gyllenberg and Silvestrov (1998, 1999a, 2000a, 2008), Englund (2000, 2001), Ni, Silvestrov and Malyarenko (2008), Ni (2010a, b, 2011, 2012, 2014), Silvestrov and Petersson (2013) and Petersson (2013a, b, 2014) have been used for getting asymptotic expansions for stationary and quasi-stationary distributions for nonlinearly perturbed Markov chains and semi-Markov processes with absorption.

Aggregation/disaggregation methods based on various modification of Gauss elimination method and space screening procedures for perturbed Markov chains have been employed for approximation of stationary distributions for Markov chains in works by Coderch, Willsky, Sastry and Castañon (1983), Delebecque (1983), Gaütsgori and Pervozvanskiu (1983), Chatelin and Miranker (1984), Courtois and Semal (1984b), Seneta (1984), Cao and Stewart (1985), Vantilborgh (1985), Feinberg and Chiu (1987), Haviv (1987, 1992, 1999), Sumita and Reiders (1988), Meyer (1989), Schweitzer (1991), Stewart and Zhang (1991), Stewart (1993a), Kim and Smith (1995) and Avrachenkov, Filar and Howlett (2013), Silvestrov, D, and Silvestrov S. (2015).

We would like to mention that the present paper contains also a more extended bibliography of works in the area supplemented by short bibliographical remarks given in the last section of the paper.

In the present paper, we combine methods based on stochastic aggregation/disaggregation approach with methods based on asymptotic expansions for perturbed regenerative processes applied to perturbed semi-Markov processes.

In the above mentioned works based on stochastic aggregation/disaggregation approach, space screening procedures for discrete time Markov chains are used. A Markov chain with a reduced phase space is constructed from the initial one as the sequence of its states at sequential moment of hitting into the reduced phase space. Times between sequential hitting of a reduced phase space are ignored. Such screening procedure preserves ratios of hitting 
frequencies for states from the reduced phase space and, thus, the ratios of stationary probabilities are the same for the initial and the reduced Markov chains. This implies that the stationary probabilities for the reduced Markov chain coincide with the corresponding stationary probabilities for the initial Markov chain up to the change of the corresponding normalizing factors.

We use another more complex type of time-space screening procedures for semi-Markov processes. In this case, a semi-Markov process with a reduced phase space is constructed from the initial one as the sequence of its states at sequential moment of hitting into the reduced phase space and times between sequential jumps of the reduced semi-Markov process are times between sequential hitting of the reduced space by the initial semi-Markov process. Such screening procedure preserves transition times between states from the reduced phase space, i.e., these times and, thus, their expectations are the same for the initial and the reduced semi-Markov processes.

We also formulate perturbation conditions in terms of asymptotic expansions for transition characteristics of perturbed semi-Markov processes. The remainders in these expansions and, thus, the transition characteristics of perturbed semi-Markov processes can be non-analytical functions of perturbation parameters that makes difference with the results for models with linear, polynomial and analytical perturbations.

We employ the methods of asymptotic analysis for nonlinearly perturbed regenerative processes developed in works by Silvestrov (1995, 2007, 2010) and Gyllenberg and Silvestrov (1998, 1999a, 2000a, 2008). However, we use techniques of more general Laurent asymptotic expansions instead of Taylor asymptotic expansions used in the above mentioned works and combine these methods with the aggregation/disaggregation approach instead of using the approach based on generalized matrix inverses. This permits us consider perturbed semi-Markov processes with an arbitrary communication structure of the phase space for the limiting semi-Markov process, including the general case, where this phase space may consist from one or several closed classes of communicative states and possibly a class of transient states.

Another new element is that we consider asymptotic expansions with remainders given not only in the form $o(\cdot)$, but, also, with explicit upper bounds.

It should be mentioned that the semi-Markov setting is an adequate and necessary element of the method proposed in the paper. Even in the case, where the initial process is a discrete or continuous time Markov chain, the time-space screening procedure of phase space reduction results in a semi- 
Markov process, since times between sequential hitting of the reduced space by the initial process have distributions which can differ of geometrical or exponential ones.

Also, the use of Laurent asymptotic expansions for expectations of sojourn times of perturbed semi-Markov processes is also a necessary element of the method. Indeed, even in the case, where expectations of sojourn times for all states of the initial semi-Markov process are asymptotically bounded and represented by Taylor asymptotic expansions, the exclusion of an asymptotically absorbing state from the initial phase space can cause appearance of states with asymptotically unbounded expectations of sojourn times represented by Laurent asymptotic expansions, for the reduced semi-Markov processes.

The method proposed in the paper can be considered as a stochastic analogue of the Gauss elimination method. It is based on the procedure of sequential exclusion of states from the phase space of a perturbed semi-Markov process accompanied by re-calculation of asymptotic expansions penetrating perturbation conditions for semi-Markov processes with reduced phase spaces. The corresponding algorithms are based on some kind of "operational calculus" for Laurent asymptotic expansions with remainders given in two forms, without or with explicit upper bounds.

The corresponding computational algorithms have an universal character. As was mentioned above, they can be applied to perturbed semi-Markov processes with an arbitrary communicative structure and are computationally effective due to recurrent character of computational procedures.

In conclusion, we would like to point out that, by our opinion, the results presented in the paper have a good potential for continuation of studies (asymptotic expansions for high order power and exponential moments for hitting times, aggregated time-space screening procedures, asymptotic expansions for quasi-stationary distributions, etc.). We comment some prospective directions for future studies in the end of the paper.

The paper includes 8 sections. In Section 2, we present so-called operational rules for Laurent asymptotic expansions. In Section 3, we formulate basic perturbation conditions for Markov chains and semi-Markov processes. In Section 4, we give basic formulas for stationary distributions for semiMarkov processes, in particular, formulas connecting stationary distributions with expectations of return times. In Section 5, we present an one-step procedure of phase space reduction for semi-Markov processes. In Section 6, we present algorithms for re-calculation of asymptotic expansions for transi- 
tion characteristics of perturbed semi-Markov processes with a reduced phase space. In Section 7, we present algorithms of sequential reduction of phase space for semi-Markov processes and construction of asymptotic expansions for stationary distributions. In Section 8, we present some directions for future studies and short bibliographical remarks concerned works in the area.

We would like to conclude the introduction with the remark that the present paper is a slightly improved version of the research report Silvestrov, D. and Silvestrov S. (2015).

\section{Laurent asymptotic expansions}

In this section, we present so-called operational rules for Laurent asymptotic expansions. We consider the corresponding results as possibly known, except, some of explicit formulas for remainders, in particular, those related to product, reciprocal and quotient rules.

2.1. Definition of Laurent asymptotic expansions. Let $A(\varepsilon)$ be a real-valued function defined on an interval $\left(0, \varepsilon_{0}\right]$, for some $0<\varepsilon_{0} \leq 1$, and given on this interval by a Laurent asymptotic expansion,

$$
A(\varepsilon)=a_{h_{A}} \varepsilon^{h_{A}}+\cdots+a_{k_{A}} \varepsilon^{k_{A}}+o_{A}\left(\varepsilon^{k_{A}}\right),
$$

where (a) $-\infty<h_{A} \leq k_{A}<\infty$ are integers, (b) the coefficients $a_{h_{A}}, \ldots, a_{k_{A}}$ are real numbers, (c) function $o_{A}\left(\varepsilon^{k_{A}}\right) / \varepsilon^{k_{A}} \rightarrow 0$ as $\varepsilon \rightarrow 0$.

We refer to such Laurent asymptotic expansion as a $\left(h_{A}, k_{A}\right)$-expansion.

We say that $\left(h_{A}, k_{A}\right)$-expansion $A(\varepsilon)$ is pivotal if it is known that $a_{h_{A}} \neq 0$.

We also say that $\left(h_{A}, k_{A}\right)$-expansion $A(\varepsilon)$ is a $\left(h_{A}, k_{A}, \delta_{A}, G_{A}, \varepsilon_{A}\right)$-expansion if its remainder $o_{A}\left(\varepsilon^{k_{A}}\right)$ satisfies the following inequalities (d) $\left|o_{A}\left(\varepsilon^{k_{A}}\right)\right|$ $\leq G_{A} \varepsilon^{k_{A}+\delta_{A}}$, for $0<\varepsilon \leq \varepsilon_{A}$, where (e) $0<\delta_{A} \leq 1,0<G_{A}<\infty$ and $0<\varepsilon_{A} \leq \varepsilon_{0}$.

In what follows, $[a]$ is the integer part of a real number $a$.

Also, the indicator of relation $A=B$ is denoted as $I(A=B)$. It equals to 1 , if $A=B$, or 0 , if $A \neq B$.

It is useful to note that there is no sense to consider, it seems, a more general case of upper bounds for the remainder $o_{A}\left(\varepsilon^{k_{A}}\right)$, with parameter $\delta_{A}>1$. Indeed, let us define $k_{A}^{\prime}=k_{A}+\left[\delta_{A}\right]-I\left(\delta_{A}=\left[\delta_{A}\right]\right)$ and $\delta_{A}^{\prime}=$ $\delta_{A}-\left[\delta_{A}\right]+I\left(\delta_{A}=\left[\delta_{A}\right]\right) \in(0,1]$.

The $\left(h_{A}, k_{A}, \delta_{A}, G_{A}, \varepsilon_{A}\right)$-expansion (11) can be re-written in the equivalent 
form of the $\left(h_{A}, k_{A}^{\prime}, \delta_{A}^{\prime}, G_{A}, \varepsilon_{A}\right)$-expansion,

$$
A(\varepsilon)=a_{h_{A}} \varepsilon^{h_{A}}+\cdots+a_{k_{A}} \varepsilon^{k_{A}}+0 \varepsilon^{k_{A}+1}+\cdots+0 \varepsilon^{k_{A}^{\prime}}+o_{A}^{\prime}\left(\varepsilon^{k_{A}^{\prime}}\right),
$$

with the remainder term $o_{A}^{\prime}\left(\varepsilon^{k_{A}^{\prime}}\right)=o_{A}\left(\varepsilon^{k_{A}}\right)$, which satisfies inequalities $\left|o_{A}^{\prime}\left(\varepsilon^{k_{A}^{\prime}}\right)\right|=\left|o_{A}\left(\varepsilon^{k_{A}}\right)\right| \leq G_{A} \varepsilon^{k_{A}+\delta_{A}}=G_{A} \varepsilon^{k_{A}^{\prime}+\delta_{A}^{\prime}}$, for $0<\varepsilon \leq \varepsilon_{A}$.

Relation (2) implies that the asymptotic expansion $A(\varepsilon)$ can be represented in different forms. In such cases, we consider a more informative form with larger parameters $h_{A}$ and $k_{A}$. As far as parameters $\delta_{A}, G_{A}$ and $\varepsilon_{A}$ are concerned, we consider as a more informative form, first, with larger value of parameter $\delta_{A}$, second, with smaller values of parameter $G_{A}$ and, third, with the larger values of parameter $\varepsilon_{A}$.

In what follows, $a \vee b=\max (a, b)$ and $a \wedge b=\min (a, b)$, for real numbers $a$ and $b$.

It is useful to note that formula (1) uniquely define part of coefficients $a_{h_{A}}, \ldots, a_{k_{A}}$.

Lemma 1. If function $A(\varepsilon)=a_{h_{A}^{\prime}}^{\prime} \varepsilon^{h_{A}^{\prime}}+\cdots+a_{k_{A}^{\prime}}^{\prime} \varepsilon_{A}^{k^{\prime}}+o_{A}^{\prime}\left(\varepsilon^{k_{A}^{\prime}}\right)=a_{h_{A}^{\prime \prime}}^{\prime \prime} \varepsilon_{A}^{h_{A}^{\prime \prime}}+$ $\cdots+a_{k_{A}^{\prime \prime}}^{\prime \prime} \varepsilon_{A}^{k_{A}^{\prime \prime}}+o_{A}^{\prime \prime}\left(\varepsilon^{k_{A}^{\prime \prime}}\right), \varepsilon \in\left(0, \varepsilon_{0}\right]$ can be represented as, respectively, $\left(h_{A}^{\prime}, k_{A}^{\prime}\right)$ and $\left(h_{A}^{\prime \prime}, k_{A}^{\prime \prime}\right)$-expansion, then the asymptotic expansion for function $A(\varepsilon)$ can be represented in the following the most informative form $A(\varepsilon)=a_{h_{A}} \varepsilon^{h_{A}}+$ $\cdots+a_{k_{A}} \varepsilon^{k_{A}}+o_{A}\left(\varepsilon^{k_{A}}\right), \varepsilon \in\left(0, \varepsilon_{0}\right]$ of $\left(h_{A}, k_{A}\right)$-expansion, with parameters $h_{A}=h_{A}^{\prime} \vee h_{A}^{\prime \prime}, k_{A}=k_{A}^{\prime} \vee k_{A}^{\prime \prime}$, and coefficients $a_{h_{A}}, \ldots, a_{k_{A}}$ and remainder $o_{A}\left(\varepsilon^{k_{A}}\right)$ given by the following relations:

(i) $a_{l}^{\prime}, a_{l}^{\prime \prime}=0$, for $l<h_{A}$.

(ii) $a_{l}=a_{l}^{\prime}=a_{l}^{\prime \prime}$, for $h_{A} \leq l \leq \tilde{k}_{A}=k_{A}^{\prime} \wedge k_{A}^{\prime \prime}$.

(iii) $a_{l}=a_{l}^{\prime \prime}$, for $\tilde{k}_{A}=k_{A}^{\prime}<l \leq k_{A}$ if $k_{A}^{\prime}<k_{A}^{\prime \prime}$.

(iv) $a_{l}=a_{l}^{\prime}$, for $\tilde{k}_{A}=k_{A}^{\prime \prime}<l \leq k_{A}$ if $k_{A}^{\prime \prime}<k_{A}^{\prime}$.

(v) The remainder term $o_{A}\left(\varepsilon^{k_{A}}\right)$ is given by the following relation,

$$
o_{A}\left(\varepsilon^{k_{A}}\right)= \begin{cases}o_{A}^{\prime \prime}\left(\varepsilon^{k_{A}^{\prime \prime}}\right) & \text { if } k_{A}^{\prime}<k_{A}^{\prime \prime}, \\ o_{A}^{\prime}\left(\varepsilon^{k_{A}^{\prime}}\right)=o_{A}^{\prime \prime}\left(\varepsilon^{k_{A}^{\prime \prime}}\right) & \text { if } k_{A}^{\prime}=k_{A}^{\prime \prime}, \\ o_{A}^{\prime}\left(\varepsilon^{k_{A}^{\prime}}\right) & \text { if } k_{A}^{\prime}>k_{A}^{\prime \prime} .\end{cases}
$$


The latter asymptotical expansion is pivotal if and only if $a_{h_{A}}=a_{h_{A}}^{\prime}=$ $a_{h_{A}}^{\prime \prime} \neq 0$.

It is useful to make some additional remarks.

The case $\tilde{k}_{A}<h_{A}$ is possible. In this case, the set of integers $l$ such that $h_{A} \leq l \leq \tilde{k}_{A}$ is empty. This can happen if $k_{A}^{\prime}<h_{A}^{\prime \prime}$ or $k_{A}^{\prime \prime}<h_{A}^{\prime}$. In the first case, all coefficients $a_{l}^{\prime}=0, l=h_{A}^{\prime}, \ldots, k_{A}^{\prime}$ while $h_{A}=h_{A}^{\prime \prime}, k=k_{A}^{\prime \prime}, a_{l}^{\prime \prime}=$ $a_{l}, l=h_{A}, \ldots, k_{A}$. In the second case, all coefficients $a_{l}^{\prime \prime}=0, l=h_{A}^{\prime \prime}, \ldots, k_{A}^{\prime \prime}$ while $h_{A}=h_{A}^{\prime}, k_{A}=k_{A}^{\prime}, a_{l}=a_{l}^{\prime}, l=h_{A}, \ldots, k_{A}$.

If $k_{A}^{\prime}=k_{A}^{\prime \prime}$ then $h_{A} \leq \tilde{k}_{A}=k_{A}$ and the set of integers $l$ such that $\tilde{k}_{A}<$ $l \leq k_{A}$ is empty. In this case, all coefficients $a_{l}=a_{l}^{\prime}=a_{l}^{\prime \prime}, l=h_{A}, \ldots, k_{A}$.

If $a_{h_{A}}^{\prime} \neq 0$ then $h_{A}=h_{A}^{\prime}$ and $a_{h_{A}}=a_{h_{A}^{\prime}}^{\prime} \neq 0$. If $a_{h_{A}^{\prime \prime}}^{\prime \prime} \neq 0$ then $h_{A}=h_{A}^{\prime \prime}$ and $a_{h_{A}}=a_{h_{A}^{\prime \prime}}^{\prime \prime} \neq 0$. If $a_{h_{A}^{\prime}}^{\prime}, a_{h_{A}^{\prime \prime}}^{\prime \prime} \neq 0$ then $h_{A}=h_{A}^{\prime}=h_{A}^{\prime \prime}$ and $a_{h_{A}}=a_{h_{A}^{\prime}}^{\prime}=a_{h_{A}^{\prime \prime}}^{\prime \prime} \neq 0$.

The following proposition supplements Lemma 1 .

Lemma 2. If $A(\varepsilon)=a_{h_{A}^{\prime}}^{\prime} \varepsilon^{h_{A}^{\prime}}+\cdots+a_{k_{A}^{\prime}}^{\prime} \varepsilon^{k_{A}^{\prime}}+o_{A}^{\prime}\left(\varepsilon^{k_{A}^{\prime}}\right)=a_{h_{A}^{\prime \prime}}^{\prime \prime} h_{A}^{h^{\prime \prime}}+\cdots+$ $a_{k_{A}^{\prime \prime}}^{\prime \prime} \varepsilon_{A}^{k_{A}^{\prime \prime}}+o_{A}^{\prime \prime}\left(\varepsilon^{k_{A}^{\prime \prime}}\right), \varepsilon \in\left(0, \varepsilon_{0}\right]$ can be represented as, respectively, $\left(h_{A}^{\prime}, k_{A}^{\prime}, \delta_{A}^{\prime}, G_{A}^{\prime}\right.$, $\varepsilon_{A}^{\prime}{ }^{A}$ - and $\left(h_{A}^{\prime \prime}, k_{A}^{\prime \prime}, \delta_{A}^{\prime \prime}, G_{A}^{\prime \prime}, \varepsilon_{A}^{\prime \prime}\right)$-expansion, then:

(i) The asymptotic expansion $A(\varepsilon)=a_{h_{A}} \varepsilon^{h_{A}}+\cdots+a_{k_{A}} \varepsilon^{k}+o_{A}\left(\varepsilon^{k_{A}}\right), \varepsilon \in$ $\left(0, \varepsilon_{0}\right]$ given in Lemma 1 is an $\left(h_{A}, k_{A}, \delta_{A}, G_{A}, \varepsilon_{A}\right)$-expansion with parameters $G_{A}, \delta_{A}$ and $\varepsilon_{A}$ which can be chosen in the following way consistent with the priority order described above:

$$
\left(\delta_{A}, G_{A}, \varepsilon_{A}\right)= \begin{cases}\left(\delta_{A}^{\prime \prime}, G_{A}^{\prime \prime}, \varepsilon_{A}^{\prime \prime}\right) & \text { if } k_{A}^{\prime}<k_{A}^{\prime \prime}, \\ \left(\delta_{A}^{\prime \prime}, G_{A}^{\prime \prime}, \varepsilon_{A}^{\prime \prime}\right) & \text { if } k_{A}^{\prime}=k_{A}^{\prime \prime}, \delta_{A}^{\prime}<\delta_{A}^{\prime \prime}, \\ \left(\delta_{A}^{\prime}=\delta_{A}^{\prime \prime}, G_{A}^{\prime} \wedge G_{A}^{\prime \prime}, \varepsilon_{A}^{\prime} \wedge \varepsilon_{A}^{\prime \prime}\right) & \text { if } k_{A}^{\prime}=k_{A}^{\prime \prime}, \delta_{A}^{\prime}=\delta_{A}^{\prime \prime}, \\ \left(\delta_{A}^{\prime}, G_{A}^{\prime}, \varepsilon_{A}^{\prime}\right) & \text { if } k_{A}^{\prime}=k_{A}^{\prime \prime}, \delta_{A}^{\prime}>\delta_{A}^{\prime \prime}, \\ \left(\delta_{A}^{\prime}, G_{A}^{\prime}, \varepsilon_{A}^{\prime}\right) & \text { if } k_{A}^{\prime}>k_{A}^{\prime \prime} .\end{cases}
$$

(ii) The asymptotic expansion $A(\varepsilon)$ can also be represented in the form $A(\varepsilon)=a_{\tilde{h}_{A}} \varepsilon^{\tilde{h}_{A}}+\cdots+a_{\tilde{k}_{A}} \tilde{\varepsilon}^{\tilde{k}_{A}}+\tilde{o}_{A}\left(\varepsilon^{\tilde{k}_{A}}\right)$ of an $\left(\tilde{h}_{A}, \tilde{k}_{A}, \tilde{\delta}_{A}, \tilde{G}_{A}, \tilde{\varepsilon}_{A}\right)-$ expansion, with parameters $\tilde{h}_{A}=h_{A}, \tilde{k}_{A}=k_{A}^{\prime} \wedge k_{A}^{\prime \prime}$ and parameters $\tilde{\delta}_{A}, \tilde{G}_{A}, \tilde{\varepsilon}_{A}$ given by the following formulas,

$$
\tilde{\delta}_{A}= \begin{cases}\delta_{A}^{\prime} & \text { if } k_{A}^{\prime}<k_{A}^{\prime \prime}, \\ \delta_{A}^{\prime} \wedge \delta_{A}^{\prime \prime} & \text { if } k_{A}^{\prime}=k_{A}^{\prime \prime}, \\ \delta_{A}^{\prime \prime} & \text { if } k_{A}^{\prime}>k_{A}^{\prime \prime},\end{cases}
$$




$$
\begin{aligned}
& \tilde{G}_{A}= \begin{cases}G_{A}^{\prime} \wedge\left(\sum_{k_{A}^{\prime}<l \leq k_{A}^{\prime \prime}}\left|a_{l}^{\prime \prime}\right| \tilde{\varepsilon}_{A}^{l-k_{A}^{\prime}-\delta_{A}^{\prime}}+G_{A}^{\prime \prime} \tilde{\varepsilon}_{A}^{\left.k_{A}^{\prime \prime}+\delta_{A}^{\prime \prime}-k_{A}^{\prime}-\delta_{A}^{\prime}\right)}\right. & \text { if } k_{A}^{\prime}<k_{A}^{\prime \prime}, \\
G_{A}^{\prime} \tilde{\varepsilon}_{A}^{\delta_{A}^{\prime}-\tilde{\delta}_{A}} \wedge G_{A}^{\prime \prime} \tilde{\varepsilon}_{A}^{\delta_{A}^{\prime \prime}-\tilde{\delta}_{A}} & \text { if } k_{A}^{\prime}=k_{A}^{\prime \prime}, \\
G_{A}^{\prime \prime} \wedge\left(\left.\sum_{k_{A}^{\prime \prime}<l \leq k_{A}^{\prime}}\left|a_{l}^{\prime}\right|\right|_{A} ^{l-k_{A}^{\prime \prime}-\delta_{A}^{\prime \prime}}+G_{A}^{\prime} \tilde{\varepsilon}_{A}^{\left.k_{A}^{\prime}+\delta_{A}^{\prime}-k_{A}^{\prime \prime}-\delta_{A}^{\prime \prime}\right)}\right. & \text { if } k_{A}^{\prime}>k_{A}^{\prime \prime} .\end{cases} \\
& \tilde{\varepsilon}_{A}=\varepsilon_{A}^{\prime} \wedge \varepsilon_{A}^{\prime \prime} .
\end{aligned}
$$

(iii) The remainders $o_{A}^{\prime}\left(\varepsilon^{k_{A}^{\prime}}\right), o_{A}^{\prime \prime}\left(\varepsilon^{k_{A}^{\prime \prime}}\right), o_{A}\left(\varepsilon^{k_{A}}\right)$ and $\tilde{o}_{A}\left(\varepsilon^{k_{A}}\right)$ are connected by the following relations:

$$
\begin{aligned}
\tilde{o}_{A}\left(\varepsilon^{\tilde{k}_{A}}\right) & =o_{A}\left(\varepsilon^{k_{A}}\right)+\sum_{\tilde{k}_{A}<l \leq k_{A}} a_{l} \varepsilon^{l} \\
& = \begin{cases}o_{A}^{\prime}\left(\varepsilon^{k_{A}^{\prime}}\right) & \text { if } k_{A}^{\prime}<k_{A}^{\prime \prime}, \\
o_{A}^{\prime}\left(\varepsilon^{k_{A}^{\prime}}\right)=o_{A}^{\prime \prime}\left(\varepsilon^{k_{A}^{\prime \prime}}\right) & \text { if } k_{A}^{\prime}=k_{A}^{\prime \prime}, \\
o_{A}^{\prime \prime}\left(\varepsilon^{k_{A}^{\prime \prime}}\right) & \text { if } k_{A}^{\prime}>k_{A}^{\prime \prime} .\end{cases}
\end{aligned}
$$

2.2. Operational rules for Laurent asymptotic expansions. Let us consider four Laurent asymptotic expansions, $A(\varepsilon)=a_{h_{A}} \varepsilon^{h_{A}}+\cdots+a_{k_{A}} \varepsilon^{k_{A}}+$ $o_{A}\left(\varepsilon^{k_{A}}\right), B(\varepsilon)=b_{h_{B}} \varepsilon^{h_{B}}+\cdots+b_{k_{B}} \varepsilon^{k_{B}}+o_{B}\left(\varepsilon^{k_{B}}\right), C(\varepsilon)=c_{h_{C}} \varepsilon^{h_{C}}+\cdots+$ $c_{k_{C}} \varepsilon^{k_{C}}+o_{C}\left(\varepsilon^{k_{C}}\right)$, and $D(\varepsilon)=d_{h_{D}} \varepsilon^{h_{D}}+\cdots+d_{k_{D}} \varepsilon^{k_{D}}+o_{D}\left(\varepsilon^{k_{D}}\right)$ defined for $0<\varepsilon \leq \varepsilon_{0}$, for some $0<\varepsilon_{0} \leq 1$.

The following lemma presents "operational" rules for Laurent asymptotic expansions.

Lemma 3. The above asymptotic expansions have the following operational rules for computing coefficients:

(i) If $A(\varepsilon), \varepsilon \in\left(0, \varepsilon_{0}\right]$ is a $\left(h_{A}, k_{A}\right)$-expansion and $c$ is a constant, then $C(\varepsilon)=c A(\varepsilon), \varepsilon \in\left(0, \varepsilon_{0}\right]$ is a $\left(h_{C}, k_{C}\right)$-expansion with parameters $h_{C}=$ $h_{A}, k_{C}=k_{A}$ and coefficients,

$$
c_{h_{C}+r}=c a_{h_{C}+r}, r=0, \ldots, k_{C}-h_{C} .
$$

This expansion is pivotal if and only if $c_{h_{C}}=c a_{h_{A}} \neq 0$.

(ii) If $A(\varepsilon), \varepsilon \in\left(0, \varepsilon_{0}\right]$ is a $\left(h_{A}, k_{A}\right)$-expansion and $B(\varepsilon), \varepsilon \in\left(0, \varepsilon_{0}\right]$ is a $\left(h_{B}, k_{B}\right)$-expansion, then $C(\varepsilon)=A(\varepsilon)+B(\varepsilon), \varepsilon \in\left(0, \varepsilon_{0}\right]$ is a $\left(h_{C}, k_{C}\right)$ expansion with parameters $h_{C}=h_{A} \wedge h_{B}, k_{C}=k_{A} \wedge k_{B}$, and coefficients,

$$
c_{h_{C}+r}=a_{h_{C}+r}+b_{h_{C}+r}, r=0, \ldots, k_{C}-h_{C},
$$


where $a_{h_{C}+r}=0$ for $0 \leq r<h_{A}-h_{C}$ and $b_{h_{C}+r}=0$ for $0 \leq r<$ $h_{B}-h_{C}$.

This expansion is pivotal if and only if $c_{h_{C}}=a_{h_{C}}+b_{h_{C}} \neq 0$.

(iii) If $A(\varepsilon), \varepsilon \in\left(0, \varepsilon_{0}\right]$ is a $\left(h_{A}, k_{A}\right)$-expansion and $B(\varepsilon), \varepsilon \in\left(0, \varepsilon_{0}\right]$ is a $\left(h_{B}, k_{B}\right)$-expansion, then $C(\varepsilon)=A(\varepsilon) \cdot B(\varepsilon), \varepsilon \in\left(0, \varepsilon_{0}\right]$ is a $\left(h_{C}, k_{C}\right)$ expansion with parameters $h_{C}=h_{A}+h_{B}, k_{C}=\left(h_{A}+k_{B}\right) \wedge\left(h_{B}+k_{A}\right)$, and coefficients,

$$
c_{h_{C}+r}=\sum_{0 \leq i \leq r} a_{h_{A}+i} b_{h_{B}+r-i}, r=0, \ldots, k_{C}-h_{C} .
$$

This expansion is pivotal if and only if $c_{h_{C}}=a_{h_{A}} b_{h_{B}} \neq 0$.

(iv) If $B(\varepsilon), \varepsilon \in\left(0, \varepsilon_{0}\right]$ is a pivotal $\left(h_{B}, k_{B}\right)$-expansion, then there exists $0<$ $\varepsilon_{0}^{\prime} \leq \varepsilon_{0}$ such that $B(\varepsilon) \neq 0, \varepsilon \in\left(0, \varepsilon_{0}^{\prime}\right]$, and $C(\varepsilon)=1 / B(\varepsilon), \varepsilon \in\left(0, \varepsilon_{0}^{\prime}\right]$ is a pivotal $\left(h_{C}, k_{C}\right)$-expansion with parameters $h_{C}=-h_{B}, k_{C}=k_{B}-2 h_{B}$ and coefficients,

$$
c_{h_{C}}=b_{h_{B}}^{-1}, c_{h_{C}+r}=-b_{h_{B}}^{-1} \sum_{1 \leq i \leq r} b_{h_{B}+i} c_{h_{C}+r-i}, r=1, \ldots, k_{C}-h_{C} .
$$

(v) If $A(\varepsilon), \varepsilon \in\left(0, \varepsilon_{0}\right]$ is a $\left(h_{A}, k_{A}\right)$-expansion $B(\varepsilon), \varepsilon \in\left(0, \varepsilon_{0}\right]$ is a pivotal $\left(h_{B}, k_{B}\right)$-expansion, then, there exists $0<\varepsilon_{0}^{\prime} \leq \varepsilon_{0}$ such that $B(\varepsilon) \neq$ $0, \varepsilon \in\left(0, \varepsilon_{0}^{\prime}\right]$, and $D(\varepsilon)=A(\varepsilon) / B(\varepsilon), \varepsilon \in\left(0, \varepsilon_{0}^{\prime}\right]$ is a $\left(h_{D}, k_{D}\right)$-expansion with parameters $h_{D}=h_{A}-h_{B}, k_{D}=\left(k_{A}-h_{B}\right) \wedge\left(h_{A}+k_{B}-2 h_{B}\right)$, and coefficients,

$$
d_{h_{D}+r}=\sum_{0 \leq i \leq r} c_{h_{C}+i} a_{h_{A}+r-i}, r=0, \ldots, k_{D}-h_{D},
$$

where $c_{h_{C}+j}, j=0, \ldots, k_{C}-h_{C}$ are coefficients of the $\left(h_{C}, k_{C}\right)$-expansion $C(\varepsilon)=1 / B(\varepsilon)$ given in the above proposition (iv), or by formulas,

$$
d_{h_{D}+r}=b_{h_{B}}^{-1}\left(a_{h_{A}+r}-\sum_{1 \leq i \leq r} b_{h_{B}+i} d_{h_{D}+r-i}\right), r=0, \ldots, k_{D}-h_{D} .
$$

This expansion is pivotal if and only if $d_{h_{D}}=a_{h_{A}} c_{h_{C}}=a_{h_{A}} / b_{h_{B}} \neq 0$. 
The following proposition presents "operational" rules for computing parameters of upper bounds for remainders of Laurent asymptotic expansions.

Lemma 4. The above asymptotic expansions have the following operational rules for computing remainders:

(i) If $A(\varepsilon), \varepsilon \in\left(0, \varepsilon_{0}\right]$ is a $\left(h_{A}, k_{A}, \delta_{A}, G_{A}, \varepsilon_{A}\right)$-expansion and $d$ is a constant, then $C(\varepsilon)=c A(\varepsilon), \varepsilon \in\left(0, \varepsilon_{0}\right]$ is a $\left(h_{C}, k_{C}, \delta_{C}, G_{C}, \varepsilon_{C}\right)$-expansion with parameters $h_{C}=h_{A}, k_{C}=k_{A}$, coefficients $c_{r}, r=h_{C}, \ldots, k_{C}$ given in proposition (i) of Lemma 3, and parameters $\delta_{C}, G_{C}, \varepsilon_{C}$ given by the following formulas,

$$
\delta_{C}=\delta_{A}, G_{C}=|c| G_{A}, \varepsilon_{C}=\varepsilon_{A} .
$$

(ii) If $A(\varepsilon), \varepsilon \in\left(0, \varepsilon_{0}\right]$ is a $\left(h_{A}, k_{A}, \delta_{A}, G_{A}, \varepsilon_{A}\right)$-expansion and $B(\varepsilon), \varepsilon \in$ $\left(0, \varepsilon_{0}\right]$ is a $\left(h_{B}, k_{B}, \delta_{B}, G_{B}, \varepsilon_{B}\right)$-expansion, then $C(\varepsilon)=A(\varepsilon)+B(\varepsilon)$, $\varepsilon \in\left(0, \varepsilon_{0}\right]$ is a $\left(h_{C}, k_{C}, \delta_{C}, G_{C}, \varepsilon_{C}\right)$-expansion with parameters $h_{C}=$ $h_{A} \wedge h_{B}, k_{C}=k_{A} \wedge k_{B}$, coefficients $c_{r}, r=h_{C}, \ldots, k_{C}$ given in proposition (ii) of Lemma 3, and parameters $\delta_{C}, G_{C}, \varepsilon_{C}$ given by the following formulas,

$$
\begin{aligned}
\delta_{C}= & \begin{cases}\delta_{A} & \text { if } k_{C}=k_{A}<k_{B}, \\
\delta_{A} \wedge \delta_{B} & \text { if } k_{C}=k_{A}=k_{B}, \\
\delta_{B} & \text { if } k_{C}=k_{B}<k_{A},\end{cases} \\
\geq & \delta_{A} \wedge \delta_{B}, \\
G_{C}= & G_{A} \varepsilon_{C}^{k_{A}+\delta_{A}-k_{C}-\delta_{C}}+\sum_{k_{C}<i \leq k_{A}}\left|a_{i}\right| \varepsilon_{C}^{i-k_{C}-\delta_{C}}
\end{aligned}
$$

(iii) If $A(\varepsilon), \varepsilon \in\left(0, \varepsilon_{0}\right]$ is a $\left(h_{A}, k_{A}, \delta_{A}, G_{A}, \varepsilon_{A}\right)$-expansion and $B(\varepsilon), \varepsilon \in$ $\left(0, \varepsilon_{0}\right]$ is a $\left(h_{B}, k_{B}, \delta_{B}, G_{B}, \varepsilon_{B}\right)$-expansion, then $C(\varepsilon)=A(\varepsilon) \cdot B(\varepsilon)$, $\varepsilon \in\left(0, \varepsilon_{0}\right]$ is a $\left(h_{C}, k_{C}, \delta_{C}, G_{C}, \varepsilon_{C}\right)$-expansion with parameters $h_{C}=$ $h_{A}+h_{B}, k_{C}=\left(h_{A}+k_{B}\right) \wedge\left(h_{B}+k_{A}\right)$, coefficients $c_{r}, r=h_{C}, \ldots, k_{C}$ 
given in proposition (iii) of Lemma 3, and parameters $\delta_{C}, G_{C}, \varepsilon_{C}$ given by the following formulas,

$$
\begin{aligned}
& \delta_{C}= \begin{cases}\delta_{A} & \text { if } k_{C}=h_{B}+k_{A}<h_{A}+k_{B}, \\
\delta_{A} \wedge \delta_{B} & \text { if } k_{C}=h_{B}+k_{A}=h_{A}+k_{B}, \\
\delta_{B} & \text { if } k_{C}=h_{A}+k_{B}<h_{B}+k_{A},\end{cases} \\
& \geq \delta_{A} \wedge \delta_{B}, \\
& G_{C}=\sum_{k_{C}<i+j, h_{A} \leq i \leq k_{A}, h_{B} \leq j \leq k_{B}}\left|a_{i}\right|\left|b_{j}\right| \varepsilon_{C}^{i+j-k_{C}-\delta_{C}} \\
& +G_{A} \sum_{h_{B} \leq j \leq k_{B}}\left|b_{j}\right| \varepsilon_{C}^{j+k_{A}+\delta_{A}-k_{C}-\delta_{C}} \\
& +G_{B} \sum_{h_{A} \leq i \leq k_{A}}\left|a_{i}\right| \varepsilon_{C}^{i+k_{B}+\delta_{B}-k_{C}-\delta_{C}} \\
& +G_{A} G_{B} \varepsilon_{C}^{k_{A}+k_{B}+\delta_{A}+\delta_{B}-k_{C}-\delta_{C}}, \\
& \varepsilon_{C}=\varepsilon_{A} \wedge \varepsilon_{B} .
\end{aligned}
$$

(iv) If $B(\varepsilon), \varepsilon \in\left(0, \varepsilon_{0}\right]$ is a pivotal $\left(h_{B}, k_{B}, \delta_{B}, G_{B}, \varepsilon_{B}\right)$-expansion, then, there exist $\varepsilon_{C} \leq \varepsilon_{0}^{\prime} \leq \varepsilon_{0}$ such that $B(\varepsilon) \neq 0, \varepsilon \in\left(0, \varepsilon_{0}^{\prime}\right]$, and $C(\varepsilon)=$ $1 / B(\varepsilon), \varepsilon \in\left(0, \varepsilon_{0}^{\prime}\right]$ is a pivotal $\left(h_{C}, k_{C}, \delta_{C}, G_{C}, \varepsilon_{C}\right)$-expansion with parameters $h_{C}=-h_{B}, k_{C}=k_{B}-2 h_{B}$, coefficients $c_{r}, r=h_{C}, \ldots, k_{C}$ given in proposition (iv) of Lemma 3, and parameters $\delta_{C}, G_{C}, \varepsilon_{C}$ given by the following formulas,

$$
\begin{aligned}
\delta_{C}= & \delta_{B} \\
G_{C}= & \left(\frac{\left|b_{h_{B}}\right|}{2}\right)^{-1}\left(\sum_{k_{B}-h_{B}<i+j, h_{B} \leq i \leq k_{B}, h_{C} \leq j \leq k_{C}}\left|b_{i}\right|\left|c_{j}\right| \varepsilon_{C}^{i+j-k_{B}+h_{B}-\delta_{B}}\right. \\
& \left.+G_{B} \sum_{h_{C} \leq j \leq k_{C}}\left|c_{j}\right| \varepsilon_{C}^{j+h_{B}}\right), \\
\varepsilon_{C}= & \varepsilon_{B} \wedge \begin{cases}\frac{\left|b_{h_{B}}\right|}{2}\left(\sum_{h_{B}<i \leq k_{B}}\left|b_{i}\right| \varepsilon_{B}^{i-h_{B}-1}\right. \\
\left.+G_{B} \varepsilon_{B}^{k_{B}+\delta_{B}-h_{B}-1}\right)^{-1} & \text { if } h_{B}<k_{B} \\
\left(\frac{\left|b_{h_{B}}\right|}{2 G_{B}}\right)^{\frac{1}{\delta_{B}}} & \text { if } h_{B}=k_{B} .\end{cases}
\end{aligned}
$$


(v) If $A(\varepsilon), \varepsilon \in\left(0, \varepsilon_{0}\right]$ is a $\left(h_{A}, k_{A}, \delta_{A}, G_{A}, \varepsilon_{A}\right)$-expansion, $B(\varepsilon), \varepsilon \in\left(0, \varepsilon_{0}\right]$ is a pivotal $\left(h_{B}, k_{B}, \delta_{B}, G_{B}, \varepsilon_{B}\right)$-expansion, then, there exist $\varepsilon_{D} \leq \varepsilon_{0}^{\prime} \leq$ $\varepsilon_{0}$ such that $B(\varepsilon) \neq 0, \varepsilon \in\left(0, \varepsilon_{0}^{\prime}\right]$, and $D(\varepsilon)=A(\varepsilon) / B(\varepsilon)$ is a $\left(h_{D}, k_{D}\right.$, $\left.\delta_{D}, G_{D}, \varepsilon_{D}\right)$-expansion with parameters $h_{D}=h_{A}+h_{C}=h_{A}-h_{B}, k_{D}=$ $\left(k_{A}+h_{C}\right) \wedge\left(h_{A}+k_{C}\right)=\left(k_{A}-h_{B}\right) \wedge\left(h_{A}+k_{B}-2 h_{B}\right)$, coefficients $d_{r}, r=h_{D}, \ldots, k_{D}$ given in proposition ( $\left.\mathbf{v}\right)$ of Lemma 3, and parameters $\delta_{D}, G_{D}, \varepsilon_{D}$ given by the following formulas,

$$
\begin{aligned}
& \delta_{D}= \begin{cases}\delta_{A} & \text { if } k_{D}=h_{C}+k_{A}<h_{A}+k_{C} \\
\delta_{A} \wedge \delta_{C} & \text { if } k_{D}=h_{C}+k_{A}=h_{A}+k_{C}, \\
\delta_{C} & \text { if } k_{D}=h_{A}+k_{C}<h_{C}+k_{A},\end{cases} \\
& \geq \delta_{A} \wedge \delta_{C}=\delta_{A} \wedge \delta_{B} \\
& G_{D}=\sum_{k_{D}<i+j, h_{A} \leq i \leq k_{A}, h_{C} \leq j \leq k_{C}}\left|a_{i}\right|\left|c_{j}\right| \varepsilon_{D}^{i+j-k_{D}-\delta_{D}} \\
& +G_{A} \sum_{h_{C} \leq j \leq k_{C}}\left|c_{j}\right| \varepsilon_{D}^{j+k_{A}+\delta_{A}-k_{D}-\delta_{D}} \\
& +G_{C} \sum_{h_{A} \leq i \leq k_{A}}\left|a_{i}\right| \varepsilon_{D}^{i+k_{C}+\delta_{C}-k_{D}-\delta_{D}} \\
& +G_{A} G_{C} \varepsilon_{D}^{k_{A}+k_{C}+\delta_{A}+\delta_{C}-k_{D}-\delta_{D}} \\
& \varepsilon_{D}=\varepsilon_{A} \wedge \varepsilon_{C},
\end{aligned}
$$

where coefficients $c_{r}, r=h_{C}, \ldots, k_{C}$ and parameters $h_{C}, k_{C}, \delta_{C}, G_{C}, \varepsilon_{C}$ are given for the $\left(h_{C}, k_{C}, \delta_{C}, G_{C}, \varepsilon_{C}\right)$-expansion of function $C(\varepsilon)=$ $1 / B(\varepsilon)$ in proposition (iv), or by formulas,

$$
\begin{aligned}
\delta_{D} & = \begin{cases}\delta_{A} & \text { if } k_{D}=k_{A}-h_{B}<h_{A}+k_{B}-2 h_{B}, \\
\delta_{A} \wedge \delta_{B} & \text { if } k_{D}=k_{A}-h_{B}=h_{A}+k_{B}-2 h_{B}, \\
\delta_{B} & \text { if } k_{D}=h_{A}+k_{B}-2 h_{B}<k_{A}-h_{B},\end{cases} \\
& \geq \delta_{A} \wedge \delta_{B},
\end{aligned}
$$




$$
\begin{aligned}
G_{D}= & \left(\frac{\left|b_{h_{B}}\right|}{2}\right)^{-1}\left(\sum_{k_{A} \wedge\left(h_{A}+k_{B}-h_{B}\right)<i \leq k_{A}}\left|a_{i}\right| \varepsilon_{D}^{i-h_{B}-k_{D}-\delta_{D}}\right. \\
& +\sum_{k_{A} \wedge\left(h_{A}+k_{B}-h_{B}\right)<i+j, h_{A} \leq i \leq k_{A}, h_{D} \leq j \leq k_{D}}\left|a_{i}\right|\left|d_{j}\right| \varepsilon_{D}^{i+j-k_{D}-h_{B}-\delta_{D}} \\
& \left.+G_{A} \varepsilon_{D}^{k_{A}+\delta_{A}-h_{B}-k_{D}-\delta_{D}}+G_{B} \sum_{h_{D} \leq j \leq k_{D}}\left|d_{j}\right| \varepsilon_{D}^{j+k_{B}+\delta_{B}-h_{B}-k_{D}-\delta_{D}}\right), \\
\varepsilon_{D}= & \varepsilon_{A} \wedge \varepsilon_{B} \wedge \begin{cases}\frac{\left|b_{h_{B}}\right|}{2}\left(\sum_{h_{B}<i \leq k_{B}}\left|b_{i}\right| \varepsilon_{B}^{i-h_{B}-1}\right. \\
\left.+G_{B} \varepsilon_{B}^{k_{B}+\delta_{B}-h_{B}-1}\right)^{-1} & \text { if } h_{B}<k_{B}, \\
\left(\frac{\left|b_{h_{B}}\right|}{2 G_{B}}\right)^{\frac{1}{\delta_{B}}} & \text { if } h_{B}=k_{B} .\end{cases}
\end{aligned}
$$

In what follows, the following two lemmas, which present recurrent operational rules for computing coefficients and remainders for multiple summations and multiplications of Laurent asymptotic expansions, will also be used. These lemmas are direct corollaries of Lemmas 3 and 4 .

Let $A_{m}(\varepsilon)=a_{h_{A_{m}, m}} \varepsilon^{h_{A_{m}}}+\cdots+a_{k_{A_{m}, m}} \varepsilon^{k_{A_{m}}}+o\left(\varepsilon^{k_{A_{m}}}\right), \varepsilon \in\left(0, \varepsilon_{0}\right]$ be a $\left(h_{A_{m}}, k_{A_{m}}\right)$-expansion, for $m=1, \ldots, N, \quad B_{n}(\varepsilon)=A_{1}(\varepsilon)+\cdots+A_{n}(\varepsilon), \varepsilon \in$ $\left(0, \varepsilon_{0}\right]$, and $C_{n}(\varepsilon)=A_{1}(\varepsilon) \times \cdots \times A_{n}(\varepsilon), \varepsilon \in\left(0, \varepsilon_{0}\right]$, for $n=1, \ldots, N$.

The following two lemmas follow, respectively, from Lemmas 3 and 4 and recurrent relations $B_{n}(\varepsilon)=B_{n-1}(\varepsilon)+A_{n}(\varepsilon), \varepsilon \in\left(0, \varepsilon_{0}\right], n=2, \ldots, N$ and $C_{n}(\varepsilon)=C_{n-1}(\varepsilon) \cdot A_{n}(\varepsilon), \varepsilon \in\left(0, \varepsilon_{0}\right], n=2, \ldots, N$, which hold for any $N \geq 2$.

Lemma 5. The above asymptotic expansions have the following operational rules for computing coefficients:

(i) If $A_{m}(\varepsilon), \varepsilon \in\left(0, \varepsilon_{0}\right]$ is a $\left(h_{A_{m}}, k_{A_{m}}\right)$-expansion for $m=1, \ldots, N$ where $N \geq 2$, then $B_{n}(\varepsilon)=b_{h_{B_{n}}, n} \varepsilon^{h_{B_{n}}}+\cdots+b_{k_{B_{n}}, n} \varepsilon^{k_{B_{n}}}+o\left(\varepsilon^{k_{B_{n}}}\right), \varepsilon \in\left(0, \varepsilon_{0}\right]$ is a $\left(h_{B_{n}}, k_{B_{n}}\right)$-expansion, for $n=1, \ldots, N$, with $h_{B_{1}}=h_{A_{1}}, k_{B_{1}}=$ $k_{A_{1}}$ and $h_{B_{n}}=\min \left(h_{A_{1}}, \ldots, h_{A_{n}}\right)=h_{B_{n-1}} \wedge h_{A_{n}}, k_{B_{n}}=\min \left(k_{A_{1}}\right.$, $\left.\ldots, k_{A_{n}}\right)=k_{B_{n-1}} \wedge k_{A_{n}}, n=2, \ldots, N$ and the coefficients given by formulas $b_{h_{B_{1}}+l, 1}=a_{h_{A_{1}}+l, 1}, l=0, \ldots, k_{B_{1}}-h_{B_{1}}=k_{A_{1}}-h_{A_{1}}$ and, for $l=0, \ldots, k_{B_{n}}-h_{B_{n}}, n=2, \ldots, N$, by formulas,

$$
b_{h_{B_{n}}+l, n}=a_{h_{B_{n}}+l, 1}+\cdots+a_{h_{B_{n}}+l, n},
$$


or

$$
b_{h_{B_{n}}+l, n}=b_{h_{B_{n-1}}+l, n-1}+a_{h_{B_{n}}+l, n},
$$

where $b_{h_{B_{n-1}}+l, n-1}=0, l=0, \ldots, h_{B_{n-1}}-h_{B_{n}}$ and $a_{h_{B_{n}}+l, m}=0, l=$ $0, \ldots h_{A_{m}}-h_{B_{n}}, m=1, \ldots, n$.

Expansions $B_{n}(\varepsilon), n=1, \ldots, N$ are pivotal if and only if $b_{h_{B_{n}}, n}=$ $a_{h_{A_{1}}, 1}+\cdots+a_{h_{A_{n}}, n} \neq 0, n=1, \ldots, N$.

(ii) If $A_{m}(\varepsilon), \varepsilon \in\left(0, \varepsilon_{0}\right]$ is a $\left(h_{A_{m}}, k_{A_{m}}\right)$-expansion for $m=1, \ldots, N$ where $N \geq 2$, then $C_{n}(\varepsilon)=c_{h_{C_{n}}, n} \varepsilon^{h_{C_{n}}}+\cdots+c_{k_{C_{n}}, n} \varepsilon^{k_{C_{n}}}+o\left(\varepsilon^{k_{C_{n}}}\right), \varepsilon \in\left(0, \varepsilon_{0}\right]$ is a $\left(h_{C_{n}}, k_{C_{n}}\right)$-expansion, for $n=1, \ldots, N$, with $h_{C_{1}}=h_{A_{1}}, k_{C_{1}}=$ $k_{A_{1}}$ and $h_{C_{n}}=h_{A_{1}}+\cdots+h_{A_{n}}=h_{C_{n-1}}+h_{A_{n}}, k_{C_{n}}=\min \left(k_{A_{l}}+\right.$ $\left.\sum_{1 \leq r \leq n, r \neq l} h_{A_{r}}, l=1, \ldots, n\right)=\left(h_{C_{n-1}}+k_{A_{n}}\right) \wedge\left(k_{C_{n-1}}+h_{A_{n}}\right), n=$ $2, \ldots, N$ and coefficients given by formulas, $c_{h_{C_{1}}+l, 1}=a_{h_{A_{1}}+l, 1}, l=$ $0, \ldots, k_{C_{1}}-h_{C_{1}}=k_{A_{1}}-h_{A_{1}}$ and, for $l=0, \ldots, k_{C_{n}}-h_{C_{n}}, n=2, \ldots, N$, by formulas,

$$
c_{h_{C_{n}}+l, n}=\sum_{l_{1}+\cdots+l_{n}=l, 0 \leq l_{i} \leq k_{A_{i}}-h_{A_{i}}, i=1, \ldots, n} \prod_{1 \leq i \leq n} a_{h_{A_{i}}+l_{i}, i},
$$

or

$$
c_{h_{C_{n}}+l, n}=\sum_{0 \leq l^{\prime} \leq l} c_{h_{C_{n-1}}+l^{\prime}, n-1} a_{h_{A_{n}}+l-l^{\prime}, n} .
$$

Expansions $C_{n}(\varepsilon), n=1, \ldots, N$ are pivotal if and only if $c_{h_{C_{n}}, n}=$ $a_{h_{A_{1}}, 1} \times \cdots \times a_{h_{A_{n}}, n} \neq 0, n=1, \ldots, N$.

(iii) Asymptotic expansions for functions $B_{n}(\varepsilon)=A_{1}(\varepsilon)+\cdots+A_{n}(\varepsilon), n=$ $1, \ldots, N$ and $C_{n}(\varepsilon)=A_{1}(\varepsilon) \times \cdots \times A_{n}(\varepsilon), n=1, \ldots, N$ are invariant with respect to any permutation, respectively, of summation and multiplication order in the above formulas (19) and (21).

Lemma 6. The above asymptotic expansions have the following operational rules for computing remainders:

(i) If $A_{m}(\varepsilon), \varepsilon \in\left(0, \varepsilon_{0}\right]$ is a $\left(h_{A_{m}}, k_{A_{m}}, \delta_{A_{m}}, G_{A_{m}}, \varepsilon_{A_{m}}\right)$-expansion for $m=$ $1, \ldots, N$ where $N \geq 2$, then $B_{n}(\varepsilon), \varepsilon \in\left(0, \varepsilon_{0}\right]$ is a $\left(h_{B_{n}}, k_{B_{n}}, \delta_{B_{n}}, G_{b_{n}}\right.$, $\left.\varepsilon_{B_{n}}\right)$-expansion, for $n=1, \ldots, N$, with parameters $h_{B_{1}}=h_{A_{1}}, k_{B_{1}}=$ 
$k_{A_{1}}$ and $h_{B_{n}}=\min \left(h_{A_{1}}, \ldots, h_{A_{n}}\right)=h_{B_{n-1}} \wedge h_{A_{n}}, k_{B_{n}}=\min \left(k_{A_{1}}\right.$, $\left.\ldots, k_{A_{n}}\right)=k_{B_{n-1}} \wedge k_{A_{n}}, n=2, \ldots, N$, coefficients $b_{h_{B_{n}}+l, n}, l=0, \ldots, k_{B_{n}}$ $-h_{B_{n}}, n=1, \ldots, N$ given in proposition (i) of Lemma 5 and parameters $G_{B_{n}}, \delta_{B_{n}}, \varepsilon_{B_{n}}, n=1, \ldots, N$ given by formulas $\delta_{B_{1}}=\delta_{A_{1}} \geq \delta_{N}^{*}=$ $\min _{1 \leq m \leq n} \delta_{A_{m}}, G_{B_{1}}=G_{A_{1}}, \varepsilon_{B_{1}}=\varepsilon_{A_{1}}$ and, for $n=2, \ldots, N$, by formulas,

$$
\delta_{B_{n}}=\min _{m \in \mathbb{K}_{n}} \delta_{A_{m}} \geq \delta_{N}^{*},
$$

where

$$
\begin{aligned}
\mathbb{K}_{n} & =\left\{m: 1 \leq m \leq n, k_{m}=\min \left(k_{1}, \ldots, k_{n}\right)\right\}, \\
G_{B_{n}} & =\sum_{1 \leq i \leq n}\left(G_{A_{i}} \varepsilon_{B_{n}}^{k_{A_{i}}+\delta_{A_{i}}-k_{B_{n}}-\delta_{B_{n}}}+\sum_{k_{B_{n}}<j \leq k_{A_{i}}}\left|a_{A_{i}, j}\right| \varepsilon_{B_{n}}^{j-k_{B_{n}}-\delta_{B_{n}}}\right), \\
\varepsilon_{B_{n}} & =\min \left(\varepsilon_{A_{1}}, \ldots, \varepsilon_{A_{n}}\right),
\end{aligned}
$$

or by alternative recurrent formulas,

$$
\begin{aligned}
\delta_{B_{n}}= & \min _{m \in \mathbb{K}_{n}} \delta_{A_{m}}= \begin{cases}\delta_{B_{n-1}} & \text { if } k_{B_{n}}=k_{B_{n-1}}<k_{A_{n}}, \\
\delta_{B_{n-1}} \wedge \delta_{A_{n}} & \text { if } k_{B_{n}}=k_{B_{n-1}}=k_{A_{n}}, \\
\delta_{A_{n}} & \text { if } k_{B_{n}}=k_{A_{n}}<k_{B_{n-1}},\end{cases} \\
\geq & \delta_{N}^{*}, \\
G_{B_{n}}= & G_{B_{n-1}} \varepsilon_{B_{B_{n}}}^{k_{B_{n-1}}+\delta_{B_{n-1}}-k_{B_{n}}-\delta_{B_{n}}}+\sum_{k_{B_{n}}<i \leq k_{B_{n-1}}}\left|b_{B_{n-1}, i}\right| \varepsilon_{B_{n}}^{i-k_{B_{n}}-\delta_{B_{n}}}
\end{aligned}
$$

(ii) If $A_{m}(\varepsilon), \varepsilon \in\left(0, \varepsilon_{0}\right]$ is a $\left(h_{A_{m}}, k_{A_{m}}, \delta_{A_{m}}, G_{A_{m}}, \varepsilon_{A_{m}}\right)$-expansion for $m=$ $1, \ldots, N$ where $N \geq 2$, then $C_{n}(\varepsilon), \varepsilon \in\left(0, \varepsilon_{0}\right]$ is a $\left(h_{C_{n}}, k_{C_{n}}, \delta_{C_{n}}, G_{C_{n}}\right.$, $\varepsilon_{C_{N}}$ )-expansion, for $n=1, \ldots, N$, with parameters $h_{C_{1}}=h_{A_{1}}, k_{C_{1}}=$ $k_{A_{1}}$ and $h_{C_{n}}=h_{C_{n-1}}+h_{A_{n}}=h_{A_{1}}+\cdots+h_{A_{n}}, k_{C_{n}}=\left(h_{C_{n-1}}+k_{A_{n}}\right) \wedge$ 
$\left(k_{C_{n-1}}+h_{A_{n}}\right)=\min _{1 \leq l \leq n}\left(k_{A_{l}}+\sum_{1 \leq r \leq n, r \neq l} h_{A_{r}}\right), n=2, \ldots, N$, coefficients $c_{h_{C_{n}}+l, n}, l=0, \ldots, k_{C_{n}}-h_{C_{n}}, n=1, \ldots, N$ given in proposition (ii) of Lemma 5 and parameters $\delta_{C_{n}}, G_{C_{n}}, \varepsilon_{C_{n}}, n=1, \ldots, N$ given by formulas $\delta_{C_{1}}=\delta_{A_{1}} \geq \delta_{N}^{*}=\min _{1 \leq m \leq n} \delta_{A_{m}}, G_{C_{1}}=G_{A_{1}}, \varepsilon_{C_{1}}=\varepsilon_{A_{1}}$ and, for $n=2, \ldots, N$, by formulas,

$$
\delta_{C_{n}}=\min _{m \in \mathbb{L}_{n}} \delta_{A_{m}} \geq \delta_{N}^{*},
$$

where

$$
\begin{aligned}
& \mathbb{L}_{n}=\left\{m: 1 \leq m \leq n,\left(k_{A_{m}}+\sum_{1 \leq r \leq n, r \neq m} h_{A_{r}}\right)\right. \\
& \left.=\min _{1 \leq l \leq n}\left(k_{A_{l}}+\sum_{1 \leq r \leq n, r \neq l} h_{A_{r}}\right)\right\} \text {, } \\
& G_{C_{n}}=\sum_{k_{C_{n}}<l_{1}+\cdots+l_{n}, h_{A_{i}} \leq l_{i} \leq k_{A_{i}}, i=1, \ldots, n} \prod_{1 \leq i \leq n}\left|a_{A_{i}, l_{i}}\right| \varepsilon_{C_{n}}^{l_{1}+\cdots+l_{n}-k_{C_{n}}-\delta_{C_{n}}} \\
& +\sum_{1 \leq j \leq n} \prod_{1 \leq i \leq n, i \neq j}\left(\sum_{h_{A_{i}} \leq l \leq k_{A_{i}}}\left|a_{A_{i}, l}\right| \varepsilon_{C_{n}}^{l}\right. \\
& \left.+G_{A_{i}} \varepsilon_{C_{n}}^{k_{A_{i}}+\delta_{A_{i}}}\right) G_{A_{j}} \varepsilon_{C_{n}}^{k_{A_{j}}+\delta_{A_{j}}-k_{C_{n}}-\delta_{C_{n}}}, \\
& \varepsilon_{C_{n}}=\min _{1 \leq i \leq n} \varepsilon_{A_{i}} \text {. }
\end{aligned}
$$

or by alternative recurrent formulas,

$$
\begin{aligned}
\delta_{C_{n}}= & \begin{cases}\delta_{C_{n-1}} & \text { if } k_{C_{n}}=h_{A_{n}}+k_{C_{n-1}}<h_{C_{n-1}}+k_{A_{n}}, \\
\delta_{A_{n}} \wedge \delta_{C_{n-1}} & \text { if } k_{C_{n}}=h_{B}+k_{A}=h_{A}+k_{B}, \\
\delta_{A_{n}} & \text { if } k_{C_{n}}=h_{C_{n-1}}+k_{A_{n}}<h_{A_{n}}+k_{C_{n-1}},\end{cases} \\
\geq & \delta_{N}^{*}, \\
G_{C_{n}}= & \sum_{k_{C_{n}}<i+j, h_{C_{n-1}} \leq i \leq k_{C_{n-1}}, h_{A_{n}} \leq j \leq k_{A_{n}}}\left|c_{C_{n-1}, i}\right|\left|a_{A_{n}, j}\right| \varepsilon_{C_{n}}^{i+j-k_{C_{n}}-\delta_{C_{n}}}
\end{aligned}
$$




$$
\varepsilon_{C_{n}}=\varepsilon_{C_{n-1}} \wedge \varepsilon_{A_{n}}
$$

(iii) Parameters $\delta_{C_{n}}, G_{C_{n}}, \varepsilon_{C_{n}}, n=1, \ldots, N$ in upper bounds for remainders in the asymptotic expansions for functions $B_{n}(\varepsilon)=A_{1}(\varepsilon)+\cdots+$ $A_{n}(\varepsilon), n=1, \ldots, N$ and $C_{n}(\varepsilon)=A_{1}(\varepsilon) \times \cdots \times A_{n}(\varepsilon), n=1, \ldots, N$ are invariant with respect to any permutation, respectively, of summation and multiplication order in the above formulas (23) and (25).

It should be noted that formulas (23) and (25) give, in general, the values, which are less or equal than the values for these constants given in alternative formulas, respectively, (24) and (26).

2.3. Proofs of Lemmas $\mathbf{1}-\mathbf{6}$. The formulas given in Lemmas 1 and 2 are quite obvious. The same relate to formulas and in propositions (i) - (ii) (the multiplication by a constant and summation rules) of Lemmas 3 and 4. They can be obtained by simple accumulation of coefficients for different powers of $\varepsilon$ and terms accumulated in the corresponding remainders, as well obvious upper bounds for absolute values of sums of terms accumulated in the corresponding remainders. Lemmas 5 and 6 are corollaries of Lemmas 3 and 4 .

Let us, therefore, give short proofs of propositions (iii) - (v) of Lemmas 3 and 4.

Multiplication of asymptotic expansions $A(\varepsilon)$ and $B(\varepsilon)$ penetrating proposition (iii) of Lemmas 3 and accumulation of coefficients for powers $\varepsilon^{l}$ for $l=h_{C}, \ldots, k_{C}$ yields the following relation,

$$
\begin{aligned}
C(\varepsilon)= & A(\varepsilon) B(\varepsilon) \\
= & \left(a_{h_{A}} \varepsilon^{h_{A}}+\cdots+a_{k_{A}} \varepsilon^{k_{A}}+o_{A}\left(\varepsilon^{k_{A}}\right)\right)\left(b_{h_{B}} \varepsilon^{h_{B}}+\cdots+b_{k_{B}} \varepsilon^{k_{B}}+o_{B}\left(\varepsilon^{k_{B}}\right)\right) \\
= & \sum_{h_{C} \leq l \leq k_{C}} a_{i} b_{j} \varepsilon^{l} \\
& +\sum_{i+j=l, h_{A} \leq i \leq k_{A}, h_{B} \leq j \leq k_{B}} a_{i} b_{j} \varepsilon^{i+j} \\
& +\sum_{h_{B} \leq j \leq k_{B}} b_{j} \varepsilon^{j} o_{A}\left(\varepsilon^{k_{A}}\right)+\sum_{h_{A} \leq i \leq k_{A}} a_{i} \varepsilon^{i} o_{B}\left(\varepsilon^{k_{B}}\right)+o_{A}\left(\varepsilon^{k_{A}}\right) o_{B}\left(\varepsilon^{k_{B}}\right) \\
= & \sum_{h_{C} \leq l \leq k_{C}} c_{l} \varepsilon^{l}+o_{C}\left(\varepsilon^{k_{C}}\right),
\end{aligned}
$$


where

$$
\begin{aligned}
o_{C}\left(\varepsilon^{k_{C}}\right)= & \sum_{k_{C}<i+j, h_{A} \leq i \leq k_{A}, h_{B} \leq j \leq k_{B}} a_{i} b_{j} \varepsilon^{i+j}+\sum_{h_{B} \leq j \leq k_{B}} b_{j} \varepsilon^{j} o_{A}\left(\varepsilon^{k_{A}}\right) \\
& +\sum_{h_{A} \leq i \leq k_{A}} a_{i} \varepsilon^{i} o_{B}\left(\varepsilon^{k_{B}}\right)+o_{A}\left(\varepsilon^{k_{A}}\right) o_{B}\left(\varepsilon^{k_{B}}\right) .
\end{aligned}
$$

Obviously,

$$
\frac{o_{C}\left(\varepsilon^{k_{C}}\right)}{\varepsilon^{k_{C}}} \rightarrow 0 \text { as } 0<\varepsilon \rightarrow 0 .
$$

It should be noted that the accumulation of coefficients for powers $\varepsilon^{l}$ can be made in (27) only up to the maximal value $l=k_{C}=\left(h_{A}+k_{B}\right) \wedge\left(h_{B}+k_{A}\right)$, because of the presence in the expression for the remainder $o_{C}\left(\varepsilon^{k_{C}}\right)$ terms $b_{h_{B}} \varepsilon^{h_{B}} O_{A}\left(\varepsilon^{k_{A}}\right)$ and $a_{h_{A}} \varepsilon^{h_{A}} O_{B}\left(\varepsilon^{k_{B}}\right)$.

Also, relation (28) readily implies relation (15), which determines parameters $\delta_{C}, G_{C}, \varepsilon_{C}$ in proposition (iii) of Lemma 4.

The assumptions of proposition (iv) in Lemma 3 imply that the following relation holds,

$$
\varepsilon^{-h_{B}} B(\varepsilon) \rightarrow b_{h_{B}} \neq 0 \text { as } 0<\varepsilon \rightarrow 0 .
$$

This relation implies that there exists $0<\varepsilon_{0}^{\prime} \leq \varepsilon_{0}$ such that $B(\varepsilon) \neq 0$ for $\varepsilon \in\left(0, \varepsilon_{0}^{\prime}\right]$, and, thus, function $C(\varepsilon)=\frac{1}{B(\varepsilon)}$ is well defined for $\varepsilon \in\left(0, \varepsilon_{0}^{\prime}\right]$.

The assumptions of proposition (iv) of Lemmas 3 also imply that,

$$
\begin{aligned}
\varepsilon^{h_{B}} C(\varepsilon) & =\frac{1}{b_{h_{B}}+b_{h_{B}+1} \varepsilon \cdots+b_{k_{B}} \varepsilon^{k_{B}-h_{B}}+o_{B}\left(\varepsilon^{k_{B}}\right) \varepsilon^{-h_{B}}} \\
& \rightarrow \frac{1}{b_{h_{B}}}=c_{h_{C}} \text { as } 0<\varepsilon \rightarrow 0 .
\end{aligned}
$$

This relation means that function $\varepsilon^{h_{B}} C(\varepsilon)$ can be represented in the form $\varepsilon^{h_{B}} C(\varepsilon)=c_{h_{C}}+o(1)$, where $c_{h_{C}}=b_{h_{B}}^{-1}$, or, equivalently, that the following representation holds,

$$
C(\varepsilon)=c_{h_{C}} \varepsilon^{-h_{B}}+C_{1}(\varepsilon), \varepsilon \in\left(0, \varepsilon_{0}^{\prime}\right],
$$

where

$$
\frac{C_{1}(\varepsilon)}{\varepsilon^{-h_{B}}} \rightarrow 0 \text { as } 0<\varepsilon \rightarrow 0 .
$$

Relations (32) and (33) prove proposition (iv) of Lemmas 3 for the case, where $h_{B}=k_{B}$ that is equivalent to the relation $h_{C}=-h_{B}=k_{C}=k_{B}-2 h_{B}$. 
Note that, in the case $h_{B}=k_{B}$, the asymptotic expansion (32) for function $C(\varepsilon)$ can not be extended. Indeed,

$$
\begin{aligned}
\varepsilon^{h_{B}-1} C_{1}(\varepsilon) & =\varepsilon^{h_{B}-1}\left(C(\varepsilon)-c_{h_{C}} \varepsilon^{-h_{B}}\right) \\
& =-\frac{c_{h_{C}}}{b_{h_{B}}+o_{B}\left(\varepsilon^{h_{B}}\right) \varepsilon^{-h_{B}}} \frac{o_{B}\left(\varepsilon^{h_{B}}\right) \varepsilon^{-h_{B}}}{\varepsilon}
\end{aligned}
$$

The term $\frac{o_{B}\left(\varepsilon^{h} B\right) \varepsilon^{-h_{B}}}{\varepsilon}$ on the right hand side in (34) has an uncertain asymptotic behaviour as $0<\varepsilon \rightarrow 0$.

Let us now assume that $h_{B}+1=k_{B}$ that is equivalent to the relation $h_{C}=-h_{B}=k_{C}-1=k_{B}-2 h_{B}-1$.

In this case, the assumptions of proposition (iv) of Lemma 3 and relations (32), (33) and (34) imply that

$$
\begin{aligned}
\varepsilon^{h_{B}-1} C_{1}(\varepsilon) & =\varepsilon^{h_{B}-1}\left(C(\varepsilon)-c_{h_{C}} \varepsilon^{-h_{B}}\right) \\
& =\frac{-b_{h_{B}+1} c_{h_{C}}-o_{B}\left(\varepsilon^{h_{B}+1}\right) \varepsilon^{-h_{B}-1} c_{h_{C}}}{b_{h_{B}}+b_{h_{B}+1} \varepsilon+o_{B}\left(\varepsilon^{h_{B}+1}\right) \varepsilon^{-h_{B}}} \\
& \rightarrow \frac{-b_{h_{B}+1} c_{h_{C}}}{b_{h_{B}}}=c_{h_{C}+1} \text { as } 0<\varepsilon \rightarrow 0 .
\end{aligned}
$$

This relation means that function $\varepsilon^{h_{B}-1} C_{1}(\varepsilon)$ can be represented in the form $\varepsilon^{h_{B}-1} C_{1}(\varepsilon)=c_{h_{C}+1}+o(1)$, where $c_{h_{C}+1}=b_{h_{B}}^{-1} b_{h_{B}+1} c_{h_{C}}$, or, equivalently, that the following representation holds,

$$
C(\varepsilon)=c_{h_{C}} \varepsilon^{-h_{B}}+c_{h_{C}+1} \varepsilon^{-h_{B}+1}+C_{2}(\varepsilon), \varepsilon \in\left(0, \varepsilon_{0}^{\prime}\right],
$$

where

$$
\frac{C_{2}(\varepsilon)}{\varepsilon^{-h_{B}+1}} \rightarrow 0 \text { as } 0<\varepsilon \rightarrow 0 .
$$

Relations (36) and (37) yields proposition (iv) of Lemmas 3 for the case, where $h_{B}+1=k_{B}$.

Note that, in the case $h_{B}+1=k_{B}$, the asymptotic expansion (36) for function $C(\varepsilon)$ can not be extended. Indeed,

$$
\begin{aligned}
\varepsilon^{h_{B}-2} C_{2}(\varepsilon) & =\varepsilon^{h_{B}-2}\left(C(\varepsilon)-c_{h_{C}} \varepsilon^{-h_{B}}-c_{h_{C}+1} \varepsilon^{-h_{B}+1}\right) \\
& =-\frac{c_{h_{C}}}{b_{h_{B}}+b_{h_{B}+1} \varepsilon+o_{B}\left(\varepsilon^{h_{B}+1}\right) \varepsilon^{-h_{B}}} \frac{o_{B}\left(\varepsilon^{h_{B}+1}\right) \varepsilon^{-h_{B}-1}}{\varepsilon} .
\end{aligned}
$$


The term $\frac{o_{B}\left(\varepsilon^{h_{B}+1}\right) \varepsilon^{-h_{B}-1}}{\varepsilon}$ on the right hand side in (38) has an uncertain asymptotic behaviour as $0<\varepsilon \rightarrow 0$.

Repeating the above arguments, we can prove that function $C(\varepsilon)$ can be represented in the form of $\left(h_{C}, k_{C}\right)$-expansion, with parameters $h_{C}, k_{C}$ and coefficients $c_{h_{C}}, \ldots, c_{k_{C}}$ given in proposition (iii) of Lemma 3 , for the general case, where $h_{B}+n=k_{B}$, or, equivalently, $h_{C}=-h_{B}=k_{C}-n=k_{B}-2 h_{B}-n$, for any $n=0,1, \ldots$

The $\left(h_{C}, k_{C}\right)$-expansion for function $C(\varepsilon)=\frac{1}{B(\varepsilon)}$ can be rewritten in the equivalent form of the following relation,

$$
1=\left(b_{h_{B}} e^{h_{B}}+\cdots+b_{k_{B}} \varepsilon^{k_{B}}+o_{B}\left(\varepsilon^{k_{B}}\right)\right)\left(c_{h_{C}}+\cdots+c_{h_{C}} \varepsilon^{k_{C}}+o_{C}\left(\varepsilon^{k_{C}}\right)\right),
$$

Proposition (iii) of Lemma 3, applied to the product on the right hand side in (39), permits to represent this product in the form of $(h, k)$-expansion with parameters $h=h_{B}+h_{C}=h_{B}-h_{B}=0$ and $k=\left(h_{B}+k_{C}\right) \wedge\left(k_{B}+h_{C}\right)=$ $\left(k_{B}-h_{B}\right) \wedge\left(k_{B}-2 h_{B}+h_{B}\right)=k_{B}-h_{B}$.

By canceling coefficient for $\varepsilon^{l}$ on the left and right hand sides in (39), for $l=0, \ldots, k_{B}-h_{B}$ and then solving equation (39) with respect to the remainder $o_{C}\left(\varepsilon^{k_{C}}\right)$ permits to find the following formula for this remainder,

$$
\begin{aligned}
o_{C}\left(\varepsilon^{k_{C}}\right)= & -\frac{\sum_{k_{B}-h_{B}<i+j, h_{B} \leq i \leq k_{B}, h_{C} \leq j \leq k_{C}} b_{i} c_{j} \varepsilon^{i+j}+\sum_{h_{C} \leq j \leq k_{C}} c_{j} \varepsilon^{j} o_{B}\left(\varepsilon^{k_{B}}\right)}{b_{h_{B}} e^{h_{B}}+\cdots+b_{k_{B}} \varepsilon^{k_{B}}+o_{B}\left(\varepsilon^{k_{B}}\right)} \\
= & -\frac{\sum_{k_{B}-h_{B}<i+j, h_{B} \leq i \leq k_{B}, h_{C} \leq j \leq k_{C}} b_{i} c_{j} \varepsilon^{i+j-h_{B}}}{b_{h_{B}}+\cdots+b_{k_{B}} \varepsilon^{k_{B}-h_{B}}+o_{B}\left(\varepsilon^{k_{B}}\right) \varepsilon^{-h_{B}}} \\
& -\frac{\sum_{h_{C} \leq j \leq k_{C}} c_{j} \varepsilon^{j-h_{B}} o_{B}\left(\varepsilon^{k_{B}}\right)}{b_{h_{B}}+\cdots+b_{k_{B}} \varepsilon^{k_{B}-h_{B}}+o_{B}\left(\varepsilon^{k_{B}}\right) \varepsilon^{-h_{B}}}
\end{aligned}
$$

The assumptions made in proposition (iv) of Lemma 4, imply that $B(\varepsilon) \neq$ 0 and the following inequality holds for $0<\varepsilon \leq \varepsilon_{C}$, where $\varepsilon_{C}$ is given in relation (16),

$$
\left|b_{h_{B}}+\cdots+b_{k_{B}} \varepsilon^{k_{B}-h_{B}}+o_{B}\left(\varepsilon^{k_{B}}\right) \varepsilon^{-h_{B}}\right| \geq \frac{\left|b_{h_{B}}\right|}{2}>0
$$

The assumptions made in proposition (iv) of Lemma 4 and inequality 
(41) finally imply that the following inequality holds, for $0<\varepsilon \leq \varepsilon_{C}$,

$$
\begin{aligned}
\left|o_{C}\left(\varepsilon^{k_{C}}\right)\right| \leq & \varepsilon^{k_{B}-2 h_{B}+\delta_{B}}\left(\frac{\left|b_{h_{B}}\right|}{2}\right)^{-1} \\
& \times\left(\sum_{k_{B}-h_{B}<i+j, h_{B} \leq i \leq k_{B}, h_{C} \leq j \leq k_{C}}\left|b_{i}\right|\left|c_{j}\right| \varepsilon_{C}^{i+j-k_{B}+h_{B}-\delta_{B}}\right. \\
& \left.+G_{B} \sum_{h_{C} \leq j \leq k_{C}}\left|c_{j}\right| \varepsilon_{C}^{j+h_{B}}\right) .
\end{aligned}
$$

This inequality proofs the proposition (iv) of Lemma 4.

The first statement of proposition (v) in Lemma 3 states that function $D(\varepsilon)$ can be represented as $\left(h_{D}, k_{D}\right)$-expansion with parameters $h_{D}, k_{D}$ and coefficients $d_{h_{d}}, \ldots, d_{k_{D}}$ given in this proposition and relation (11). It is the direct corollary of propositions (iii) and (iv) of Lemma 3, which, just, should be applied to the product $D(\varepsilon)=A(\varepsilon) \cdot \frac{1}{B(\varepsilon)}, \varepsilon \in\left(0, \varepsilon_{0}^{\prime}\right]$.

Note that, in this case, parameters $h_{D}=h_{A}+h_{C}=h_{A}-h_{B}$ and $k_{D}=$ $\left(k_{A}+h_{C}\right) \wedge\left(h_{A}+k_{C}\right)=\left(k_{A}-h_{B}\right) \wedge\left(h_{A}+k_{B}-2 h_{B}\right)$.

Now, when it is already proved that $D(\varepsilon)$ is $\left(h_{D}, k_{D}\right)$-expansion, its coefficients can be also computed by equalising coefficients for for powers $\varepsilon^{l}$ for $l=h_{D}, \ldots, k_{D}$ on the left and right hand sides of relation,

$$
\begin{aligned}
A(\varepsilon)= & B(\varepsilon) D(\varepsilon) \\
= & \left(b_{h_{B}} e^{h_{B}}+\cdots+b_{h_{B}} \varepsilon^{k_{B}}+o_{B}\left(\varepsilon^{k_{B}}\right)\right) \\
& \times\left(d_{h_{D}} e^{h_{D}}+\cdots+d_{h_{D}} \varepsilon^{k_{D}}+o_{D}\left(\varepsilon^{k_{D}}\right)\right) .
\end{aligned}
$$

This procedure yields the second statement of proposition (v) in Lemma 3 and the corresponding formulas given in relation (12).

The first statement of proposition proposition ( $\mathbf{v}$ ) in Lemma 4 and relations (17) can be obtained by direct application of propositions (iii) and (iv) and relations (15) and (16) given in Lemma 4, to the product $D(\varepsilon)=$ $A(\varepsilon) \cdot \frac{1}{B(\varepsilon)}$.

Proposition (iii) of Lemma 3, applied to the product on the right hand side in (43), permits to represent this product in the form of $(h, k)$-expansion with parameters $h=h_{B}+h_{D}=h_{B}+h_{A}-h_{B}=h_{A}$ and $k=\left(h_{B}+k_{D}\right) \wedge\left(k_{B}+\right.$ $\left.h_{D}\right)=\left(h_{B}+\left(k_{A}-h_{B}\right) \wedge\left(h_{A}+k_{B}-2 h_{B}\right)\right) \wedge\left(k_{B}+h_{A}-h_{B}\right)=k_{A} \wedge\left(k_{B}+h_{A}-h_{B}\right)$.

By canceling coefficient for $\varepsilon^{l}$ on the left and right hand sides in (43), for $l=h_{A}, \ldots, k_{A} \wedge\left(k_{B}+h_{A}-h_{B}\right)$ and then solving equation (43) with respect 
to the remainder $o_{D}\left(\varepsilon^{k_{D}}\right)$ yields the following formula for this remainder,

$$
\begin{aligned}
o_{D}\left(\varepsilon^{k_{D}}\right)= & \frac{\sum_{k_{A} \wedge\left(k_{B}+h_{A}-h_{B}\right)<l \leq k_{A}} a_{l} \varepsilon^{l}+o_{A}\left(\varepsilon^{k_{A}}\right)}{b_{h_{B}} e^{h_{B}}+\cdots+b_{k_{B}} \varepsilon^{k_{B}}+o_{B}\left(\varepsilon^{k_{B}}\right)} \\
& -\frac{\sum_{k_{A} \wedge\left(k_{B}+h_{A}-h_{B}\right)<i+j, h_{B} \leq i \leq k_{B}, h_{D} \leq j \leq k_{D}} b_{i} d_{j} \varepsilon^{i+j}}{b_{h_{B}} e^{h_{B}}+\cdots+b_{k_{B}} \varepsilon^{k_{B}}+o_{B}\left(\varepsilon^{k_{B}}\right)} \\
& -\frac{\sum_{h_{D} \leq j \leq k_{D}} d_{j} \varepsilon^{j} o_{B}\left(\varepsilon^{k_{B}}\right)}{b_{h_{B}} e^{h_{B}}+\cdots+b_{k_{B}} \varepsilon^{k_{B}}+o_{B}\left(\varepsilon^{k_{B}}\right)} \\
= & \frac{\sum_{k_{A} \wedge\left(k_{B}+h_{A}-h_{B}\right)<l \leq k_{A}} a_{l} \varepsilon^{l-h_{B}}+o_{A}\left(\varepsilon^{k_{A}}\right) \varepsilon^{-h_{B}}}{b_{h_{B}}+\cdots+b_{k_{B}} \varepsilon^{k_{B}-h_{B}}+o_{B}\left(\varepsilon^{k_{B}}\right) \varepsilon^{-h_{B}}} \\
& -\frac{\sum_{k_{A} \wedge\left(k_{B}+h_{A}-h_{B}\right)<i+j, h_{B} \leq i \leq k_{B}, h_{D} \leq j \leq k_{D}} b_{i} d_{j} \varepsilon^{i+j-h_{B}}}{b_{h_{B}}+\cdots+b_{k_{B}} \varepsilon^{k_{B}-h_{B}}+o_{B}\left(\varepsilon^{k_{B}}\right) \varepsilon^{-h_{B}}} \\
& -\frac{\sum_{h_{D} \leq j \leq k_{D}} d_{j} \varepsilon^{j-h_{B}} o_{B}\left(\varepsilon^{k_{B}}\right)}{b_{h_{B}}+\cdots+b_{k_{B}} \varepsilon^{k_{B}-h_{B}}+o_{B}\left(\varepsilon^{k_{B}}\right) \varepsilon^{-h_{B}}}
\end{aligned}
$$

The assumptions made in proposition (v) of Lemma 4 and inequality (41) finally imply that the following inequality holds, for $0<\varepsilon \leq \varepsilon_{D}$ given in relation (18),

$$
\begin{aligned}
\left|o_{D}\left(\varepsilon^{k_{D}}\right)\right| \leq & \varepsilon^{k_{D}+\delta_{D}}\left(\frac{\left|b_{h_{B}}\right|}{2}\right)^{-1} \\
& \times\left(\sum_{k_{A} \wedge\left(k_{B}+h_{A}-h_{B}\right)<l \leq k_{A}}\left|a_{l}\right| \varepsilon_{D}^{l-k_{D}-h_{B}-\delta_{D}}\right. \\
& +\sum_{k_{A} \wedge\left(k_{B}+h_{A}-h_{B}\right)<i+j, h_{B} \leq i \leq k_{B}, h_{D} \leq j \leq k_{D}}\left|b_{i}\right|\left|d_{j}\right| \varepsilon_{D}^{i+j-k_{D}-h_{B}-\delta_{D}} \\
& +G_{A} e_{D}^{k_{A}+\delta_{A}-h_{B}-k_{D}-\delta_{D}} \\
& \left.+G_{B} \sum_{h_{D} \leq j \leq k_{D}}\left|d_{j}\right| \varepsilon_{D}^{j+k_{B}+\delta_{B}-h_{B}-k_{D}-\delta_{D}}\right) .
\end{aligned}
$$

2.4. Algebraic and related properties of operational rules for Laurent asymptotic expansions. Let us also introduce parameter $w_{A}=$ $k_{A}-h_{A}$, which is a length of a Laurent asymptotic expansion $A(\varepsilon)=a_{h} \varepsilon^{h_{A}}+$ $\cdots+a_{k} \varepsilon^{k_{A}}+o_{A}\left(\varepsilon^{k_{A}}\right)$.

The following useful lemma takes place.

Lemma 7. The following relations hold for Laurent asymptotic expansions penetrating Lemma 3: 
(i) If $C(\varepsilon)=c A(\varepsilon)$, then $w_{C}=w_{A}$.

(ii) If $C(\varepsilon)=A(\varepsilon)+B(\varepsilon)$, then $w_{A} \wedge w_{B} \leq w_{C} \leq w_{A} \vee w_{B}$.

(iii) If $C(\varepsilon)=A(\varepsilon) \cdot B(\varepsilon)$, then $w_{C}=w_{A} \wedge w_{B}$.

(iv) If $C(\varepsilon)=1 / B(\varepsilon)$, then $w_{C}=w_{B}$.

(v) If $D(\varepsilon)=A(\varepsilon) / B(\varepsilon)$, then $w_{D}=w_{A} \wedge w_{B}$.

(vi) If $w_{A}=w_{B}=w$ then $w_{C}=w_{D}=w$ for all Laurent asymptotic expansions penetrating Lemma 3.

The proof of this simple lemma readily follows from formulas for parameters $h$ and $k$ penetrating propositions (i) - (v) of Lemma 3 .

Let us again consider four Laurent asymptotic expansions, $A(\varepsilon)=a_{h_{A}} \varepsilon^{h_{A}}+$ $\cdots+a_{k_{A}} \varepsilon^{k_{A}}+o_{A}\left(\varepsilon^{k_{A}}\right), B(\varepsilon)=b_{h_{B}} \varepsilon^{h_{B}}+\cdots+b_{k_{B}} \varepsilon^{k_{B}}+o_{B}\left(\varepsilon^{k_{B}}\right), C(\varepsilon)=$ $c_{h_{C}} \varepsilon^{h_{C}}+\cdots+c_{k_{C}} \varepsilon^{k_{C}}+o_{C}\left(\varepsilon^{k_{C}}\right)$, and $D(\varepsilon)=d_{h_{D}} \varepsilon^{h_{D}}+\cdots+d_{k_{D}} \varepsilon^{k_{D}}+o_{D}\left(\varepsilon^{k_{D}}\right)$ defined for $0<\varepsilon \leq \varepsilon_{0}$, for some $0<\varepsilon_{0} \leq 1$.

Below, sums $\sum_{l=h}^{k} d_{l}$ are counted as 0 if $k<h$.

The following lemma is also a corollary of Lemma 3.

Lemma 8. The summation and multiplication operations for the Laurent asymptotic expansions penetrating propositions (ii) and (iii) in Lemma 3 possess the following algebraic properties, which should be understood as identities for the corresponding asymptotic expansions:

(i) The summation operation is commutative, i.e., $C(\varepsilon)=A(\varepsilon)+B(\varepsilon)=$ $B(\varepsilon)+A(\varepsilon)$, where $h_{C}=h_{A+B}=h_{B+A}=h_{A} \wedge h_{B}, k_{C}=k_{A+B}=$ $k_{B+A}=k_{A} \wedge k_{B}$, and,

$$
C(\varepsilon)=\sum_{l=0}^{k_{C}-h_{C}}\left(a_{h_{C}+l}+b_{h_{C}+l}\right) \varepsilon^{h_{C}+l}+o_{C}\left(\varepsilon^{k_{c}}\right),
$$

where $a_{h_{C}+l}=0$ for $0 \leq l<h_{A}-h_{C}, b_{h_{C}+l}=0$ for $0 \leq l<h_{B}-h_{C}$.

(ii) The summation operation is associative, i.e., $D(\varepsilon)=(A(\varepsilon)+B(\varepsilon))+$ $C(\varepsilon)=A(\varepsilon)+(B(\varepsilon)+C(\varepsilon))=A(\varepsilon)+B(\varepsilon)+C(\varepsilon)$, where $h_{D}=$ $h_{(A+B)+C}=h_{A+(B+C)}=h_{A+B+C}=h_{A} \wedge h_{B} \wedge h_{C}, k_{D}=k_{(A+B)+C}=$ $k_{A+(B+C)}=k_{A+B+C}=k_{A} \wedge k_{B} \wedge k_{C}$, and,

$$
D(\varepsilon)=\sum_{l=0}^{k_{D}-h_{D}}\left(a_{h_{D}+l}+b_{h_{D}+l}+c_{h_{D}+l}\right) \varepsilon^{h_{D}+l}+o_{D}\left(\varepsilon^{k_{D}}\right),
$$


where $a_{h_{D}+l}=0$ for $0 \leq l<h_{A}-h_{D}, b_{h_{D}+l}=0$ for $0 \leq l<h_{B}-h_{D}$, $c_{h_{D}+l}=0$ for $0 \leq l<h_{C}-h_{D}$.

(iii) The multiplication operation is commutative, i.e., $C(\varepsilon)=A(\varepsilon) \cdot B(\varepsilon)=$ $B(\varepsilon) \cdot A(\varepsilon)$, where $h_{C}=h_{A \cdot B}=h_{B \cdot A}=h_{A}+h_{B}, k_{C}=k_{A \cdot B}=k_{B \cdot A}=$ $\left(h_{A}+k_{B}\right) \wedge\left(k_{A}+h_{B}\right)$, and,

$$
C(\varepsilon)=\sum_{l=0}^{k_{C}-h_{C}}\left(\sum_{l_{1}+l_{2}=l, l_{1}, l_{2} \geq 0} a_{h_{A}+l_{1}} b_{h_{B}+l_{2}}\right) \varepsilon^{h_{C}+l}+o_{C}\left(\varepsilon^{k_{c}}\right) .
$$

(iv) The multiplication operation is associative, i.e., $D(\varepsilon)=(A(\varepsilon) \cdot B(\varepsilon))$. $C(\varepsilon)=A(\varepsilon) \cdot(B(\varepsilon) \cdot C(\varepsilon))=A(\varepsilon) \cdot B(\varepsilon) \cdot C(\varepsilon)$, where $h_{D}=h_{(A \cdot B) \cdot C}=$ $h_{A \cdot(B \cdot C)}=h_{A \cdot B \cdot C}=h_{A}+h_{B}+h_{C}, k_{D}=k_{(A \cdot B) \cdot C}=k_{A \cdot(B \cdot C)}=k_{A \cdot B \cdot C}=$ $\left(h_{A}+h_{B}+k_{C}\right) \wedge\left(h_{A}+k_{B}+h_{C}\right) \wedge\left(k_{A}+h_{B}+h_{C}\right)$, and,

$$
D(\varepsilon)=\sum_{l=0}^{k_{D}-h_{D}}\left(\sum_{l_{1}+l_{2}+l_{3}=l, l_{1}, l_{2}, l_{3} \geq 0} a_{h_{A}+l_{1}} b_{h_{B}+l_{2}} c_{h_{C}+l_{3}}\right) \varepsilon^{h_{D}+l}+o_{D}\left(\varepsilon^{k_{D}}\right) .
$$

(v) The summation and multiplication operations possess distributive property, i.e., $D(\varepsilon)=(A(\varepsilon)+B(\varepsilon)) \cdot C(\varepsilon)=A(\varepsilon) \cdot C(\varepsilon)+B(\varepsilon) \cdot C(\varepsilon)$, where $h_{D}=h_{(A+B) \cdot C}=h_{A \cdot C+B \cdot C}=h_{A} \wedge h_{B}+h_{C}=\left(h_{A}+h_{C}\right) \wedge\left(h_{B}+h_{C}\right), k_{D}=$ $k_{(A+B) \cdot C}=k_{A \cdot C+B \cdot C}=\left(h_{A} \wedge h_{B}+k_{C}\right) \wedge\left(k_{A} \wedge k_{B}+h_{C}\right)=\left(h_{A}+k_{C}\right) \wedge$ $\left(k_{A}+h_{C}\right) \wedge\left(h_{B}+k_{C}\right) \wedge\left(k_{B}+h_{C}\right)$, and,

$$
\begin{aligned}
D(\varepsilon)= & \sum_{l=0}^{k_{D}-h_{D}}\left(\sum _ { l _ { 1 } + l _ { 2 } = l , l _ { 1 } , l _ { 2 } \geq 0 } \left(a_{h_{A} \wedge h_{B}+l_{1}}\right.\right. \\
& \left.+b_{\left.h_{A} \wedge h_{B}+l_{1}\right)} c_{h_{C}+l_{2}}\right) \varepsilon^{h_{D}+l}+o_{D}\left(\varepsilon^{k_{D}}\right) \\
= & \sum_{l=0}^{k_{D}-h_{A}-h_{C}}\left(\sum_{l_{1}+l_{2}=l, l_{1}, l_{2} \geq 0} a_{h_{A}+l_{1}} c_{h_{C}+l_{2}}\right) \varepsilon^{h_{A}+h_{C}+l} \\
& +\sum_{l=0}^{k_{D}-h_{B}-h_{C}}\left(\sum_{l_{1}+l_{2}=l, l_{1}, l_{2} \geq 0} b_{h_{B}+l_{1}} c_{h_{C}+l_{2}}\right) \varepsilon^{h_{B}+h_{C}+l}+o_{D}\left(\varepsilon^{k_{D}}\right) .
\end{aligned}
$$

where $a_{h_{A} \wedge h_{B}+l}=0$. for $0 \leq l<h_{A}-h_{A} \wedge h_{B}, b_{h_{A} \wedge h_{B}+l}=0$, for $0 \leq l<h_{B}-h_{A} \wedge h_{B}$.

The summation and multiplication rules for computing of upper bounds remainders penetrating propositions (ii) and (iii) in Lemma 4 possess the 
communicative property. This follows from formulas (23) and (25) given in Lemma 6.

However, the summation and multiplication rules for computing of upper bounds for remainders presented in propositions (ii) and (iii) of Lemma 4 do not possess associative and distributional properties. The question about the form of upper bounds for the corresponding remainders, which would possess these properties, remains open.

As follows from Lemma 4, operational rules presented in this lemma possess special property that let one give an effective low bounds for parameter $\delta_{A}$ for any $\left(h_{A}, k_{A}, \delta_{A}, G_{A}, \varepsilon_{A}\right)$-expansion $A(\varepsilon)$ obtained as the result of a finite sequence of operations (multiplication by a constant, summation, multiplication, and division) performed with with $\left(h_{A_{i}}, k_{A_{i}}, \delta_{A_{i}}, G_{A_{i}}, \varepsilon_{A_{i}}\right)$-expansions $A_{i}(\varepsilon), i=1, \ldots, N$ from some finite set of such expansions.

The following lemma takes place.

Lemma 9. The operational rules for computing remainders of asymptotic expansions with explicit upper bounds for remainders presented in propositions (ii) and (iii) of Lemma 4 possess the following properties:

(i) If $C(\varepsilon)=A(\varepsilon)+B(\varepsilon)=B(\varepsilon)+A(\varepsilon)$ then $\delta_{C}=\delta_{A+B}=\delta_{B+A}, G_{C}=$ $G_{A+B}=G_{B+A}$ and $\varepsilon_{C}=\varepsilon_{A+B}=\varepsilon_{B+A}$, where parameters $\delta_{C}, G_{C}$ and $\varepsilon_{C}$ are given by formula (14) in proposition (ii) of Lemma 4.

(ii) If $C(\varepsilon)=A(\varepsilon) \cdot B(\varepsilon)=B(\varepsilon) \cdot A(\varepsilon)$ then $\delta_{C}=\delta_{A \cdot B}=\delta_{B \cdot A}, G_{C}=$ $G_{A \cdot B}=G_{B \cdot A}$ and $\varepsilon_{C}=\varepsilon_{A \cdot B}=\varepsilon_{B \cdot A}$, where parameters $\delta_{C}, G_{C}$ and $\varepsilon_{C}$ are given by formula (15) in proposition (iii) of Lemma 4 .

(iii) If $A(\varepsilon)$ is $\left(h_{A}, k_{A}, \delta_{A}, G_{A}, \varepsilon_{A}\right)$-expansion obtained as the result of a finite sequence of operations (multiplication by a constant, summation, multiplication, and quotient) performed with $\left(h_{A_{i}}, k_{A_{i}}, \delta_{A_{i}}, G_{A_{i}}, \varepsilon_{A_{i}}\right)$-expansions $A_{i}(\varepsilon), i=1, \ldots, N$ from some finite set of such expansions, then $\delta_{A} \geq \delta_{N}^{*}=\min _{1 \leq i \leq N} \delta_{A_{i}}$ that makes it possible to rewrite $A(\varepsilon)$ as the $\left(h_{A}, k_{A}, \delta_{N}^{*}, G_{A, N}^{*}, \varepsilon_{A}\right)$-expansion, with parameter $G_{A, N}^{*}=G_{A} \varepsilon_{A}^{\delta_{A}-\delta_{N}^{*}}$.

\section{Perturbed Markov chains and semi-Markov processes}

In this section, we formulate basic perturbation conditions for Markov chains and semi-Markov processes.

3.1. Perturbed Markov chains. Let $\mathbb{X}=\{1, \ldots, N\}$ and $\eta_{n}^{(\varepsilon)}, n=$ $0,1, \ldots$ be, for every $\varepsilon \in\left(0, \varepsilon_{0}\right]$, a homogeneous Markov chain with a phase 
space $\mathbb{X}$, an initial distribution $p_{i}^{(\varepsilon)}=\mathrm{P}\left\{\eta_{0}^{(\varepsilon)}=i\right\}, i \in \mathbb{X}$ and transition probabilities defined for $i, j \in \mathbb{X}$,

$$
p_{i j}(\varepsilon)=\mathrm{P}\left\{\eta_{n+1}^{(\varepsilon)}=j / \eta_{n}^{(\varepsilon)}=i\right\} .
$$

Let us assume that the following condition holds:

A: There exist sets $\mathbb{Y}_{i} \subseteq \mathbb{X}, i \in \mathbb{X}$ and $\varepsilon_{0} \in(0,1]$ such that: (a) probabilities $p_{i j}(\varepsilon)>0, j \in \mathbb{Y}_{i}, i \in \mathbb{X}$, for $\varepsilon \in\left(0, \varepsilon_{0}\right]$; (b) probabilities $p_{i j}(\varepsilon)=0, j \in \overline{\mathbb{Y}}_{i}, i \in \mathbb{X}$, for $\varepsilon \in\left(0, \varepsilon_{0}\right] ; \quad$ (c) there exist $n_{i j} \geq 1$ and a chain of states $i=l_{i j, 0}, l_{i j, 1}, \ldots, l_{i j, n_{i j}}=j$ such that $l_{i j, 1} \in$ $\mathbb{Y}_{l_{i j, 0}}, \ldots, l_{i j, n_{i j}} \in \mathbb{Y}_{l_{i j, n_{i j}-1}}$, for every pair of states $i, j \in \mathbb{X}$.

We refer to sets $\mathbb{Y}_{i}, i \in \mathbb{X}$ as transition sets.

Conditions $\mathbf{A}$ implies that all sets $\mathbb{Y}_{i} \neq \emptyset, i \in \mathbb{X}$, since matrix $\left\|p_{i j}(\varepsilon)\right\|$ is stochastic, for every $\varepsilon \in\left(0, \varepsilon_{0}\right]$.

We now assume that the following perturbation condition holds:

B: $p_{i j}(\varepsilon)=\sum_{l=l_{i j}^{-}}^{l_{i j}^{+}} a_{i j}[l] \varepsilon^{l}+o_{i j}\left(\varepsilon^{l_{i j}^{+}}\right)$, where $a_{i j}\left[l_{i j}^{-}\right]>0$ and $0 \leq l_{i j}^{-} \leq l_{i j}^{+}<$ $\infty$, for $j \in \mathbb{Y}_{i}, i \in \mathbb{X}$, and $o_{i j}\left(\varepsilon^{l_{i j}^{+}}\right) / \varepsilon^{l_{i j}^{+}} \rightarrow 0$ as $\varepsilon \rightarrow 0$, for $j \in \mathbb{Y}_{i}, i \in \mathbb{X}$.

Some additional conditions should be imposed on parameters $\varepsilon_{0} \in(0,1]$ and $l_{i j}^{ \pm}, j \in \mathbb{Y}_{i}, i \in \mathbb{X}$, and coefficients $a_{i j}[l], l=l_{i j}^{-}, \ldots, l_{i j}^{+}, j \in \mathbb{Y}_{i}, i \in \mathbb{X}$, penetrating the asymptotic expansions condition $\mathbf{B}$, in order this condition would be consistent with the model assumption that matrix $\left\|p_{i j}(\varepsilon)\right\|$ is stochastic, for every $\varepsilon \in\left(0, \varepsilon_{0}\right]$, and with condition $\mathbf{A}$.

Condition $\mathbf{B}$ implies that there exits $\varepsilon_{0} \in(0,1]$ such that the following relation holds,

$$
p_{i j}(\varepsilon)=\sum_{l=l_{i j}^{-}}^{l_{i j}^{+}} a_{i j}[l] \varepsilon^{l}+o_{i j}\left(\varepsilon^{l_{i j}^{+}}\right)>0, j \in \mathbb{Y}_{i}, i \in \mathbb{X}, \varepsilon \in\left(0, \varepsilon_{0}\right] .
$$

Thus, condition $\mathbf{B}$ is consistent with condition A (a).

The model assumption that matrix $\left\|p_{i j}(\varepsilon)\right\|$ is stochastic is, under conditions $\mathbf{A}(\mathbf{a})$ and (b), equivalent to the following relation,

$$
\sum_{j \in \mathbb{Y}_{i}} p_{i j}(\varepsilon)=1, j \in \mathbb{Y}_{i}, i \in \mathbb{X}, \varepsilon \in\left(0, \varepsilon_{0}\right]
$$


Condition $\mathbf{B}$ and proposition (i) (the multiple summation rule) of Lemma 5 imply that sum $\sum_{j \in \mathbb{Z}} p_{i j}(\varepsilon)$ can, for every subset $\mathbb{Z} \subseteq \mathbb{Y}_{i}$ and $i \in \mathbb{X}$, be represented in the form of the following asymptotic expansion,

$$
\sum_{j \in \mathbb{Z}} p_{i j}(\varepsilon)=\sum_{l=\check{l}_{i, \mathbb{Z}}^{-}}^{l_{i, \mathbb{Z}}^{+}} a_{i, \mathbb{Z}}[l] \varepsilon^{l}+o_{i, \mathbb{Z}}\left(\varepsilon^{l_{i, \mathbb{Z}}^{+}}\right)
$$

where

$$
\begin{gathered}
l_{i, \mathbb{Z}}^{-}=\min _{j \in \mathbb{Z}} l_{i j}^{-}, l_{i, \mathbb{Z}}^{+}=\min _{j \in \mathbb{Z}} l_{i j}^{+}, \\
a_{i, \mathbb{Z}}[l]=\sum_{j \in \mathbb{Z}} a_{i j}[l], l=l_{i, \mathbb{Z}}^{-}, \ldots, l_{i, \mathbb{Z}}^{+},
\end{gathered}
$$

where $a_{i j}[l]=0$, for $0 \leq l<l_{i j}^{-}, j \in \mathbb{Z}$, and

$$
o_{i, \mathbb{Z}}\left(\varepsilon^{l_{i, \mathbb{Z}}^{+}}\right)=\sum_{j \in \mathbb{Z}}\left(\sum_{l_{i, \mathbb{Z}}^{+}<l \leq l_{i j}^{+}} a_{i j}[l] \varepsilon^{l}+o_{i j}\left(\varepsilon^{l_{i j}^{+}}\right)\right) .
$$

In terms of asymptotic expansions, constant 1 can be represented, for every $n=0,1, \ldots$, in the form of the following pivotal $(0, n)$-expansion,

$$
1=1+0 \varepsilon+\cdots 0 \varepsilon^{n}+o_{n}\left(\varepsilon^{n}\right)
$$

where remainders $o_{n}\left(\varepsilon^{n}\right) \equiv 0, n=0,1, \ldots$

Moreover, the above expansion is a $\left(0, n, 1, G, \varepsilon_{0}\right)$-expansion for any $0<$ $G<\infty$ and $n=0,1, \ldots$

Relation (53) permits us apply Lemma 2 to the asymptotic expansions given in relations (54) and (58). Not that, in this case, $l_{i, \mathbb{Y}_{i}}^{-}=0$, otherwise the expression on the right hand side in (54) would converge to zero as $\varepsilon \rightarrow 0$. Let us take $n=l_{i, \mathbb{Y}_{i}}^{+}$in relation (58). In this case $h_{1}=0$ and $k_{1}=l_{i, \mathbb{Y}_{i}}^{+}$in the asymptotic expansion given in relation (58).

Lemma 2 and the model stochasticity assumption (53) imply that, in this case, the following condition should hold for the coefficients of asymptotic expansions penetrating condition $\mathbf{B}$ :

C: (a) $a_{i, \mathbb{Y}_{i}}[l]=\sum_{j \in \mathbb{Y}_{i}} a_{i j}[l]=\mathrm{I}(l=0), 0 \leq l \leq l_{i, \mathbb{Y}_{i}}^{+}, i \in \mathbb{X}$, where $a_{i j}[l]=$ 0 , for $0 \leq l<l_{i j}^{-}, j \in \mathbb{Y}_{i}, i \in \mathbb{X} ;$ (b) $o_{i, \mathbb{Y}_{i}}\left(\varepsilon^{l_{i, \mathbb{Y}_{i}}^{+}}\right) \equiv o_{l_{i, \mathbb{Y}_{i}}^{+}}\left(\varepsilon^{l_{i, \mathbb{Y}_{i}}^{+}}\right) \equiv 0, i \in \mathbb{X}$. 
Remark 1. It is possible to prove that conditions $\mathbf{A}-\mathbf{C}$ and the model stochasticity assumption (53) imply that the asymptotic expansion in (54) satisfy, for every $\mathbb{Z} \subseteq \mathbb{Y}_{i}$ and $i \in \mathbb{X}$, one of the following additional conditions: (a) $l_{i, \mathbb{Z}}^{-}>0$; (b) $l_{i, \mathbb{Z}}^{-}=0, a_{i, \mathbb{Z}}[0]<1$; (c) $l_{i, \mathbb{Z}}^{-}=0, a_{i, \mathbb{Z}}[0]=1$ and there exists $0<l_{i, \mathbb{Z}} \leq l_{i, \mathbb{Z}}^{+}$such that $a_{i, \mathbb{Z}}[l]=0,0<l<l_{i, \mathbb{Z}}$, but $a_{i, \mathbb{Z}}\left[l_{i, \mathbb{Z}}\right]<0$; or $(\mathrm{d})$ $l_{i, \mathbb{Z}}^{-}=0, a_{i, \mathbb{Z}}[0]=1$ and $a_{i, \mathbb{Z}}[l]=0,0<l \leq l_{i, \mathbb{Z}}^{+}$, but the remainder $o_{i, \mathbb{Z}}\left(\varepsilon^{l_{i, \mathbb{Z}}^{+}}\right)$is a nonpositive function of $\varepsilon$.

The above proposition implies that there exists $\varepsilon_{0} \in(0,1]$ such that $\sum_{l=l_{i, \mathbb{Z}}^{-}}^{l_{i, \mathbb{Z}}^{+}} a_{i, \mathbb{Z}}[l] \varepsilon^{l}+o_{i, \mathbb{Z}}\left(\varepsilon^{l_{i, \mathbb{Z}}^{+}}\right) \leq 1, \mathbb{Z} \subseteq \mathbb{Y}_{i}, i \in \mathbb{X}, \varepsilon \in\left(0, \varepsilon_{0}\right]$. Thus, conditions A - $\mathbf{C}$ are also consistent with the relations $\sum_{j \in \mathbb{Z}} p_{i j}(\varepsilon) \leq 1, \mathbb{Z} \subseteq \mathbb{Y}_{i}, i \in \mathbb{X}, \varepsilon \in$ $\left(0, \varepsilon_{0}\right]$, which follows from the model stochasticity assumption (153).

In the case, where the asymptotic expansions penetrating condition $\mathbf{B}$ are supposed to be given in the form of asymptotic expansions with explicit upper bounds for remainders, we replace it by the following condition:

$\mathbf{B}^{\prime}: p_{i j}(\varepsilon)=\sum_{l=l_{i j}^{-}}^{l_{i j}^{+}} a_{i j}[l] \varepsilon^{l}+o_{i j}\left(\varepsilon^{l_{i j}^{+}}\right)$, where $a_{i j}\left[l_{i j}^{-}\right]>0$ and $0 \leq l_{i j}^{-} \leq l_{i j}^{+}<$ $\infty$, for $j \in \mathbb{Y}_{i}, i \in \mathbb{X}$, and $\left|o_{i j}\left(\varepsilon^{l_{i j}^{+}}\right)\right| \leq G_{i j} \varepsilon^{l_{i j}^{+}+\delta_{i j}}, 0<\varepsilon \leq \varepsilon_{i j}$, for $j \in \mathbb{Y}_{i}, i \in \mathbb{X}$, where $0<\delta_{i j} \leq 1,0<G_{i j}<\infty$ and $0<\varepsilon_{i j} \leq \varepsilon_{0}$.

3.2. Perturbed semi-Markov processes. Let $\mathbb{X}=\{1, \ldots, N\}$ and $\left(\eta_{n}^{(\varepsilon)}, \kappa_{n}^{(\varepsilon)}\right), n=0,1, \ldots$ be, for every $\varepsilon \in(0,1]$, a Markov renewal process, i.e., a homogeneous Markov chain with the phase space $\mathbb{X} \times[0, \infty)$, an initial distribution $p_{i}^{(\varepsilon)}=\mathrm{P}\left\{\eta_{0}^{(\varepsilon)}=i, \kappa_{0}^{(\varepsilon)}=0\right\}=\mathrm{P}\left\{\eta_{0}^{(\varepsilon)}=i\right\}, i \in \mathbb{X}$ and transition probabilities defined for $(i, s),(j, t) \in \mathbb{X} \times[0, \infty)$,

$$
Q_{i j}^{(\varepsilon)}(t)=\mathrm{P}\left\{\eta_{n+1}^{(\varepsilon)}=j, \kappa_{n+1}^{(\varepsilon)} \leq t / \eta_{n}^{(\varepsilon)}=i, \kappa_{n}^{(\varepsilon)}=s\right\}
$$

In this case, the random sequence $\eta_{n}^{(\varepsilon)}, n=0,1, \ldots$ is also a homogeneous (embedded) Markov chain with the phase space $\mathbb{X}$ and transition probabilities defined for $i, j \in \mathbb{X}$,

$$
p_{i j}(\varepsilon)=\mathrm{P}\left\{\eta_{n+1}^{(\varepsilon)}=j / \eta_{n}^{(\varepsilon)}=i\right\}=Q_{i j}^{(\varepsilon)}(\infty) .
$$

We assume that condition A holds. This implies that Markov chain $\eta_{n}^{(\varepsilon)}$ has one class of communicative states, for every $\varepsilon \in\left(0, \varepsilon_{0}\right]$.

We also assume that the following condition excluding instant transitions holds: 
D: $Q_{i j}^{(\varepsilon)}(0)=0, i, j \in \mathbb{X}$, for every $\varepsilon \in\left(0, \varepsilon_{0}\right]$.

Let us now introduce a semi-Markov process,

$$
\eta^{(\varepsilon)}(t)=\eta_{\nu^{(\varepsilon)}(t)}^{(\varepsilon)}, t \geq 0 .
$$

where

$$
\nu^{(\varepsilon)}(t)=\max \left(n \geq 0: \zeta_{n}^{(\varepsilon)} \leq t\right), t \geq 0,
$$

is a number of jumps in the time interval $[0, t]$, and

$$
\zeta_{n}^{(\varepsilon)}=\kappa_{1}^{(\varepsilon)}+\cdots+\kappa_{n}^{(\varepsilon)}, n=0,1, \ldots,
$$

are sequential moments of jumps for the semi-Markov process $\eta^{(\varepsilon)}(t)$.

If $Q_{i j}^{(\varepsilon)}(t)=\left(1-e^{-\lambda_{i}(\varepsilon) t}\right) p_{i j}(\varepsilon), t \geq 0, i, j \in \mathbb{X}$, then $\eta^{(\varepsilon)}(t), t \geq 0$ is a continuous time homogeneous Markov chain.

If $Q_{i j}^{(\varepsilon)}(t)=I(t \geq 1) p_{i j}(\varepsilon), t \geq 0, i, j \in \mathbb{X}$, then $\eta^{(\varepsilon)}(t)=\eta_{[t]}^{(\varepsilon)}, t \geq 0$ is a discrete time homogeneous Markov chain embedded in continuous time.

Let us also introduce expectations of sojourn times,

$$
e_{i j}(\varepsilon)=\mathrm{E}_{i} \kappa_{1}^{(\varepsilon)} I\left(\eta_{1}^{(\varepsilon)}=j\right)=\int_{0}^{\infty} t Q_{i j}^{(\varepsilon)}(d t), i, j \in \mathbb{X} .
$$

We also assume that the following condition holds:

E: $e_{i j}(\varepsilon)<\infty, i, j \in \mathbb{X}$, for $\varepsilon \in\left(0, \varepsilon_{0}\right]$.

Here and henceforth, notations $\mathrm{P}_{i}$ and $\mathrm{E}_{i}$ are used for conditional probabilities and expectations under condition $\eta_{0}^{(\varepsilon)}=i$.

In the case of continuous time Markov chain, $e_{i j}(\varepsilon)=\frac{1}{\lambda_{i}(\varepsilon)} p_{i j}(\varepsilon), i, j \in \mathbb{X}$.

In the case of discrete time Markov chain, $e_{i j}(\varepsilon)=p_{i j}(\varepsilon), i, j \in \mathbb{X}$.

Conditions $\mathbf{A}$ and $\mathbf{D}$ imply that, for every $\varepsilon \in\left(0, \varepsilon_{0}\right]$, expectations $e_{i j}(\varepsilon)>0$, for $j \in \mathbb{Y}_{i}, i \in \mathbb{X}$, and $e_{i j}(\varepsilon)=0$, for $j \in \overline{\mathbb{Y}}_{i}, i \in \mathbb{X}$.

We now assume that the following perturbation condition holds:

$\mathbf{F}: e_{i j}(\varepsilon)=\sum_{l=m_{i j}^{-}}^{m_{i j}^{+}} b_{i j}[l] \varepsilon^{l}+\dot{o}_{i j}\left(\varepsilon^{m_{i j}^{+}}\right)$, where $b_{i j}\left[m_{i j}^{-}\right]>0$ and $-\infty<m_{i j}^{-} \leq$ $m_{i j}^{+}<\infty$, for $j \in \mathbb{Y}_{i}, i \in \mathbb{X}$ and $\dot{o}_{i j}\left(\varepsilon^{m_{i j}^{+}}\right) / \varepsilon^{m_{i j}^{+}} \rightarrow 0$ as $\varepsilon \rightarrow 0$, for $j \in \mathbb{Y}_{i}, i \in \mathbb{X}$. 
In particular, in the case of discrete time Markov chain, condition $\mathbf{B}$ implies condition $\mathbf{F}$ to hold, since, in this case, expectations $e_{i j}(\varepsilon)=p_{i j}(\varepsilon), j \in$ $\mathbb{Y}_{i}, i \in \mathbb{X}$.

Condition $\mathbf{F}$ implies that there exits $\varepsilon_{0} \in(0,1]$ such that the following relation holds,

$$
e_{i j}(\varepsilon)>0, j \in \mathbb{Y}_{i}, i \in \mathbb{X}, \varepsilon \in\left(0, \varepsilon_{0}\right] .
$$

This is consistent with condition $\mathbf{D}$.

In the case, where the asymptotic expansions penetration condition $\mathbf{F}$ are given in the form of asymptotic expansions with explicit upper bounds for remainders, we assumer that the following condition holds:

$$
\begin{aligned}
\mathbf{F}^{\prime}: & e_{i j}(\varepsilon)=\sum_{l=m_{i j}^{-}}^{m_{i j}^{+}} b_{i j}[l] \varepsilon^{l}+\dot{o}_{i j}\left(\varepsilon^{m_{i j}^{+}}\right), \text {where } b_{i j}\left[l_{i j}^{-}\right]>0 \text { and }-\infty<\leq m_{i j}^{-} \leq \\
& m_{i j}^{+}<\infty \text {, for } j \in \mathbb{Y}_{i}, i \in \mathbb{X} \text {, and }\left|\dot{o}_{i j}\left(\varepsilon^{l_{i j}^{+}}\right)\right| \leq \dot{G}_{i j} \varepsilon^{m_{i j}^{+}+\dot{\delta}_{i j}}, 0<\varepsilon \leq \dot{\varepsilon}_{i j}, \text { for } \\
& j \in \mathbb{Y}_{i}, i \in \mathbb{X}, \text { where } 0<\dot{\delta}_{i j} \leq 1,0<\dot{G}_{i j}<\infty \text { and } 0<\dot{\varepsilon}_{i j} \leq \varepsilon_{0} .
\end{aligned}
$$

It is also worse to note that the perturbation conditions $\mathbf{B}$ and $\mathbf{F}$ are independent.

To see this, let us take arbitrary functions $p_{i j}(\varepsilon), j \in \mathbb{Y}_{i}, i \in \mathbb{X}$ and $e_{i j}(\varepsilon), j \in \mathbb{Y}_{i}, i \in \mathbb{X}$ satisfying, respectively, conditions $\mathbf{B}$ and $\mathbf{F}$, and, also, relations (52), (53) and (65). Then, there exist semi-Markov transition probabilities $Q_{i j}^{(\varepsilon)}(t), t \geq 0, j \in \mathbb{Y}_{i}, i \in \mathbb{X}$ such that $Q_{i j}^{(\varepsilon)}(\infty)=p_{i j}(\varepsilon), j \in \mathbb{Y}_{i}, i \in \mathbb{X}$ and $\int_{0}^{\infty} t Q_{i j}^{(\varepsilon)}(d t)=e_{i j}(\varepsilon), j \in \mathbb{Y}_{i}, i \in \mathbb{X}$, for every $\varepsilon \in\left(0, \varepsilon_{0}\right]$.

It is readily seen that, for example, semi-Markov transition probabilities $Q_{i j}^{(\varepsilon)}(t)=I\left(t \geq e_{i j}(\varepsilon) / p_{i j}(\varepsilon)\right) p_{i j}(\varepsilon), t \geq 0, j \in \mathbb{Y}_{i}, i \in \mathbb{X}$ satisfy the above relations.

\section{Stationary distributions for semi-Markov processes}

In this section, we give basic formulas for stationary distributions for semiMarkov processes, in particular, formulas connecting stationary distributions with expectations of return times.

4.1. Stationary distributions for semi-Markov processes. Condition $\mathbf{A}$ guarantees that the phase space $\mathbb{X}$ is one class of communicative states for Markov chain $\eta_{n}^{(\varepsilon)}$, for every $\varepsilon \in\left(0, \varepsilon_{0}\right]$, i.e., the Markov chain $\eta_{n}^{(\varepsilon)}$ is ergodic, and, thus, for every $\varepsilon \in\left(0, \varepsilon_{0}\right]$, there exist the unique stationary 
distribution $\bar{\rho}(\varepsilon)=\left\langle\rho_{1}(\varepsilon), \ldots, \rho_{N}(\varepsilon)\right\rangle$, which is given by the following ergodic relation,

$$
\bar{\mu}_{i, n}^{(\varepsilon)}=\frac{1}{n} \sum_{k=1}^{n} I\left(\eta_{k}^{(\varepsilon)}=i\right) \stackrel{\text { a.s. }}{\longrightarrow} \rho_{i}(\varepsilon) \text { as } n \rightarrow \infty, i \in \mathbb{X} .
$$

It is useful to note that the ergodic relation (66) holds for any initial distribution $\bar{p}^{(\varepsilon)}=\left\langle p_{1}^{(\varepsilon)}, \ldots p_{N}^{(\varepsilon)}\right\rangle$ and the stationary distribution $\bar{\rho}(\varepsilon)$ does not depend on the initial distribution.

As known, $\rho_{i}(\varepsilon), i \in \mathbb{X}$ is the unique positive solution for the system of linear equations,

$$
\left\{\begin{array}{l}
\rho_{j}(\varepsilon)=\sum_{i \in \mathbb{X}} \rho_{i}(\varepsilon) p_{i j}(\varepsilon), j \in \mathbb{X} \\
\sum_{i \in \mathbb{X}} \rho_{i}=1
\end{array}\right.
$$

Conditions $\mathbf{A}, \mathbf{D}$ and $\mathbf{E}$ imply that, for every $\varepsilon \in\left(0, \varepsilon_{0}\right]$, the semi-Markov process $\eta^{(\varepsilon)}(t)$ is also ergodic and its stationary distribution $\bar{\pi}(\varepsilon)=\left\langle\pi_{1}(\varepsilon), \ldots\right.$, $\left.\pi_{N}(\varepsilon)\right\rangle$ is given by the following ergodic relation, for $i \in \mathbb{X}$,

$$
\bar{\mu}_{i}^{(\varepsilon)}(t)=\frac{1}{t} \int_{0}^{t} I\left(\eta^{(\varepsilon)}(s)=i\right) d s \stackrel{\text { a.s. }}{\longrightarrow} \pi_{i}(\varepsilon) \text { as } t \rightarrow \infty .
$$

As in (66), the ergodic relation (68) holds for any initial distribution $\bar{p}^{(\varepsilon)}$ and the stationary distribution $\bar{\pi}(\varepsilon)$ does not depend on the initial distribution.

The stationary distributions for the semi-Markov process $\eta^{(\varepsilon)}(t)$ and the embedded Markov chain $\eta_{n}^{(\varepsilon)}$ are connected by the following relation,

$$
\pi_{i}(\varepsilon)=\frac{\rho_{i}(\varepsilon) e_{i}(\varepsilon)}{\sum_{j \in \mathbb{X}} \rho_{j}(\varepsilon) e_{j}(\varepsilon)}, i \in \mathbb{X}
$$

where

$$
e_{i}(\varepsilon)=\mathrm{E}_{i} \kappa_{1}^{(\varepsilon)}=\sum_{j \in \mathbb{X}} e_{i j}(\varepsilon), i \in \mathbb{X} .
$$

Condition B implies that there exist limits,

$$
p_{i j}(0)=\lim _{\varepsilon \rightarrow 0} p_{i j}(\varepsilon)=\left\{\begin{array}{cl}
a_{i j}[0] & \text { if } l_{i j}^{-}=0, j \in \mathbb{Y}_{i}, i \in \mathbb{X} \\
0 & \text { if } l_{i j}^{-}>0, j \in \mathbb{Y}_{i}, i \in \mathbb{X} \\
0 & \text { if } j \in \overline{\mathbb{Y}}_{i}, i \in \mathbb{X}
\end{array}\right.
$$


Matrix $\left\|p_{i j}(\varepsilon)\right\|$ is stochastic, for every $\varepsilon \in\left(0, \varepsilon_{0}\right]$ and, thus, matrix $\left\|p_{i j}(0)\right\|$ is also stochastic.

However, it is possible that matrix $\left\|p_{i j}(0)\right\|$ has more zero elements than matrices $\left\|p_{i j}(\varepsilon)\right\|$.

Therefore, a Markov chain $\eta_{n}^{(0)}, n=0,1, \ldots$, with the phase space $\mathbb{X}$ and the matrix of transition probabilities $\left\|p_{i j}(0)\right\|$ can be not ergodic, and its phase space $\mathbb{X}$ can consist of one or several closed classes of communicative states plus, possibly, a class of transient states.

Condition $\mathbf{F}$ implies that there exist limits,

$$
e_{i j}(0)=\lim _{\varepsilon \rightarrow 0} e_{i j}(\varepsilon)=\left\{\begin{array}{cl}
\infty & \text { if } m_{i j}^{-}<0, j \in \mathbb{Y}_{i}, i \in \mathbb{X} \\
b_{i j}[0] & \text { if } m_{i j}^{-}=0, j \in \mathbb{Y}_{i}, i \in \mathbb{X} \\
0 & \text { if } m_{i j}^{-}>0, j \in \mathbb{Y}_{i}, i \in \mathbb{X} \\
0 & \text { if } j \in \overline{\mathbb{Y}}_{i}, i \in \mathbb{X}
\end{array}\right.
$$

Out goal is to design an effective algorithm for constructing asymptotic expansions for stationary probabilities $\pi_{i}(\varepsilon), i \in \mathbb{X}$, under assumption that conditions $\mathbf{A}-\mathbf{F}$ hold.

As we shall se, the proposed algorithm, based on a special techniques of sequential phase space reduction, can be applied for models with asymptotically coupled and uncoupled phase spaces and all types of asymptotic behavior of expected sojourn times.

The models of continuous and discrete Markov chains are particular cases.

In particular, asymptotic expansions for stationary probabilities $\rho_{i}(\varepsilon), i \in$ $\mathbb{X}$ coincide with expansions for stationary probabilities $\pi_{i}(\varepsilon), i \in \mathbb{X}$, for the discrete time Markov chain, where expectations $e_{i j}(\varepsilon)=p_{i j}(\varepsilon), i, j \in \mathbb{X}$.

4.2. Expected hitting times and stationary probabilities for semi-Markov processes. Let us define hitting times, which are random variables given by the following relation, for $j \in \mathbb{X}$,

$$
\tau_{j}^{(\varepsilon)}=\sum_{n=1}^{\nu_{j}^{(\varepsilon)}} \kappa_{n}^{(\varepsilon)},
$$

where

$$
\nu_{j}^{(\varepsilon)}=\min \left(n \geq 1: \eta_{n}^{(\varepsilon)}=j\right) .
$$

Let us denote,

$$
E_{i j}(\varepsilon)=\mathrm{E}_{i} \tau_{j}^{(\varepsilon)}, i, j \in \mathbb{X}
$$


As is known, conditions $\mathbf{A}, \mathbf{D}$ and $\mathbf{E}$ imply that, for every $\varepsilon \in\left(0, \varepsilon_{0}\right]$,

$$
0<E_{i j}(\varepsilon)<\infty, i, j \in \mathbb{X}
$$

Moreover, under the above conditions, the expectations $E_{i j}(\varepsilon), i \in \mathbb{X}$ are, for every $j \in \mathbb{X}$, the unique solution for the following system of linear equations,

$$
\left\{E_{i j}(\varepsilon)=e_{i}(\varepsilon)+\sum_{r \neq j} p_{i r}(\varepsilon) E_{r j}(\varepsilon), i \in \mathbb{X} .\right.
$$

The following relation plays an important role in what follows,

$$
\pi_{i}(\varepsilon)=\frac{e_{i}(\varepsilon)}{E_{i i}(\varepsilon)}, i \in \mathbb{X} .
$$

In fact this formula is an alternative form of relation (69). Indeed, as is known, $E_{i i}(\varepsilon)=\sum_{j \in \mathbb{X}} e_{j}(\varepsilon) f_{i i, j}(\varepsilon)$, where $f_{i i, j}(\varepsilon)$ is the expected number of visits by the Markov chain $\eta_{n}^{(\varepsilon)}$ the state $j$ between two sequential visits of the state $i$. As also known, $f_{i i, j}(\varepsilon)=\rho_{j}(\varepsilon) / \rho_{i}(\varepsilon), i, j \in \mathbb{X}$.

Formula (78) permits reduce the problem of constructing asymptotic expansions for semi-Markov stationary probabilities $\pi_{i}(\varepsilon)$ to the problem of constructing Laurent asymptotic expansions for expectation of hitting times $E_{i i}(\varepsilon)$.

\section{Semi-Markov processes with reduced phase spaces}

In this section, we present a procedure for one-step procedure of phase space reduction for semi-Markov processes.

5.1. Reduction of a phase space for semi-Markov process. Let us choose some state $r$ and consider the reduced phase space ${ }_{r} \mathbb{X}=\{i \in \mathbb{X}, i \neq$ $r$, with the state $r$ excluded from the phase space $\mathbb{X}$.

Let us assume that $p_{r}^{(\varepsilon)}=\mathrm{P}\left\{\eta_{0}^{(\varepsilon)}=r\right\}=0$ and define the sequential moments of hitting the reduced space ${ }_{r} \mathbb{X}$ by the embedded Markov chain $\eta_{n}^{(\varepsilon)}$

$$
{ }_{r} \xi_{n}^{(\varepsilon)}=\min \left(k>{ }_{r} \xi_{n-1}^{(\varepsilon)}, \eta_{k}^{(\varepsilon)} \in{ }_{r} \mathbb{X}\right), n=1,2, \ldots,{ }_{r} \xi_{0}^{(\varepsilon)}=0 .
$$

Now, let us define the random sequence,

$$
\left({ }_{r} \eta_{n}^{(\varepsilon)},{ }_{r} \kappa_{n}^{(\varepsilon)}\right)= \begin{cases}\left(\eta_{r \xi_{n}^{(\varepsilon)}}^{(\varepsilon)}, \sum_{k={ }_{r} \xi_{n-1}^{(\varepsilon)} \xi_{1}^{(\varepsilon)}} \kappa_{k}^{(\varepsilon)}\right) & \text { for } n=1,2, \ldots \\ \left(\eta_{0}^{(\varepsilon)}, 0\right) & \text { for } n=0 .\end{cases}
$$


This sequence is also a Markov renewal process with a phase space ${ }_{r} \mathbb{X} \times$ $[0, \infty)$, the initial distribution $p_{i}^{(\varepsilon)}=\mathrm{P}\left\{\eta_{0}^{(\varepsilon)}=i\right\}, i \in{ }_{r} \mathbb{X}$ (remind that $p_{r}^{(\varepsilon)}=$ $0)$, and transition probabilities defined for $(i, s),(j, t) \in{ }_{r} \mathbb{X} \times[0, \infty)$,

$$
{ }_{r} Q_{i j}^{(\varepsilon)}(t)=\mathrm{P}\left\{{ }_{r} \eta_{n+1}^{(\varepsilon)}=j,{ }_{r} \kappa_{n+1}^{(\varepsilon)} \leq t /{ }_{r} \eta_{n}^{(\varepsilon)}=i,{ }_{r} \kappa_{n}^{(\varepsilon)}=s\right\} .
$$

Respectively, one can define the transformed semi-Markov process with the reduced phase space ${ }_{r} \mathbb{X}$,

$$
{ }_{r} \eta^{(\varepsilon)}(t)={ }_{r} \eta_{r \nu^{(\varepsilon)}(t)}^{(\varepsilon)}, \quad t \geq 0 .
$$

where

$$
{ }_{r} \nu^{(\varepsilon)}(t)=\max \left(n \geq 0:{ }_{r} \zeta_{n}^{(\varepsilon)} \leq t\right), t \geq 0,
$$

is a number of jumps at time interval $[0, t]$, and

$$
{ }_{r} \zeta_{n}^{(\varepsilon)}={ }_{r} \kappa_{1}^{(\varepsilon)}+\cdots+{ }_{r} \kappa_{n}^{(\varepsilon)}, n=0,1, \ldots,
$$

are sequential moments of jumps for the semi-Markov process ${ }_{r} \eta^{(\varepsilon)}(t)$.

The transition probabilities ${ }_{r} Q_{i j}^{(\varepsilon)}(t)$ are expressed via the transition probabilities $Q_{i j}^{(\varepsilon)}(t)$ by the following formula, for $i, j \in{ }_{r} \mathbb{X}, t \geq 0$,

$$
{ }_{r} Q_{i j}^{(\varepsilon)}(t)=Q_{i j}^{(\varepsilon)}(t)+\sum_{n=0}^{\infty} Q_{i r}^{(\varepsilon)}(t) * Q_{r r}^{(\varepsilon) * n}(t) * Q_{r j}^{(\varepsilon)}(t) .
$$

Here, symbol $*$ is used to denote a convolution of distribution functions (possibly improper) and $Q_{r r}^{(\varepsilon) * n}(t)$ is the $n$ times convolution of the distribution function $Q_{r r}^{(\varepsilon)}(t)$ given by the following recurrent formula, for $r \in \mathbb{X}$,

$$
Q_{r r}^{(\varepsilon) * n}(t)=\left\{\begin{array}{cl}
\int_{0}^{t} Q_{r r}^{(\varepsilon) *(n-1)}(t-s) Q_{r r}^{(\varepsilon)}(d s) & \text { for } t \geq 0 \text { and } n \geq 1 \\
I(t \geq 0) & \text { for } t \geq 0 \text { and } n=0
\end{array}\right.
$$

Relation (85) directly implies the following formula for transition probabilities of the embedded Markov chain ${ }_{r} \eta_{n}^{(\varepsilon)}$, for $i, j \in{ }_{r} \mathbb{X}$,

$$
\begin{aligned}
{ }_{r} p_{i j}(\varepsilon) & ={ }_{r} Q_{i j}^{(\varepsilon)}(\infty) \\
& =p_{i j}(\varepsilon)+\sum_{n=0}^{\infty} p_{i r}(\varepsilon) p_{r r}(\varepsilon)^{n} p_{r j}(\varepsilon) \\
& =p_{i j}(\varepsilon)+p_{i r}(\varepsilon) \frac{p_{r j}(\varepsilon)}{1-p_{r r}(\varepsilon)} .
\end{aligned}
$$


Let us denote,

$$
\mathbb{Y}_{i r}^{+}=\left\{j \in \mathbb{Y}_{i}: j \neq r\right\}, i, r \in \mathbb{X}
$$

and

$$
\mathbb{Y}_{i r}^{-}=\left\{j \in{ }_{r} \mathbb{X}: r \in \mathbb{Y}_{i}, j \in \mathbb{Y}_{r}\right\}, i \in{ }_{r} \mathbb{X}
$$

Condition $\mathbf{A}$ implies that sets $\mathbb{Y}_{r r}^{+} \neq \emptyset, r \in \mathbb{X}$.

Thus, probabilities $1-p_{r r}(\varepsilon)>0, r \in \mathbb{X}$, for every $\varepsilon \in\left(0, \varepsilon_{0}\right]$.

That is why,

$$
\begin{aligned}
{ }_{r} \mathbb{Y}_{i} & =\left\{j \in{ }_{r} \mathbb{X}:{ }_{r} p_{i j}(\varepsilon)>0, \varepsilon \in\left(0, \varepsilon_{0}\right]\right\} \\
& =\left\{j \in{ }_{r} \mathbb{X}: j \in \mathbb{Y}_{i}\right\} \cup\left\{j \in{ }_{r} \mathbb{X}: r \in \mathbb{Y}_{i}, j \in \mathbb{Y}_{r}\right\} \\
& =\mathbb{Y}_{i r}^{+} \cup \mathbb{Y}_{i r}^{-}, i \in{ }_{r} \mathbb{X}
\end{aligned}
$$

Relation (87) and condition A, assumed to hold for the Markov chain $\eta_{n}^{(\varepsilon)}$, imply that condition $\mathbf{A}$ also holds for the Markov chain ${ }_{r} \eta_{n}^{(\varepsilon)}$, with the sets ${ }_{r} \mathbb{Y}_{i}, i \in{ }_{r} \mathbb{X}$.

Indeed, let $i \in{ }_{r} \mathbb{X}$. If $j \in \mathbb{Y}_{i r}^{+}$then $p_{i j}(\varepsilon)>0$ and, thus, ${ }_{r} p_{i j}(\varepsilon)>0$. If $j \in \mathbb{Y}_{i r}^{-}$then $p_{i r}(\varepsilon), p_{r j}(\varepsilon)>0$ and, again, ${ }_{r} p_{i j}(\varepsilon)>0$. If $j \notin \mathbb{Y}_{i r}^{+} \cup \mathbb{Y}_{i r}^{-}$ then $p_{i j}(\varepsilon)=0$ and $p_{i r}(\varepsilon), p_{r j}(\varepsilon)=0$. By relation (87), this implies that ${ }_{r} p_{i j}(\varepsilon)=0$.

Let $i \in{ }_{r} \mathbb{X}$. If $\mathbb{Y}_{i r}^{+} \neq \emptyset$ then ${ }_{r} \mathbb{Y}_{i} \neq \emptyset$. If $\mathbb{Y}_{i r}^{+}=\emptyset$ then $r \in \mathbb{Y}_{i}$ and, thus, $p_{i r}(\varepsilon)>0$. Then, $\mathbb{Y}_{i r}^{-}=\left\{j \in{ }_{r} \mathbb{X}: p_{r j}(\varepsilon)>0\right\}=\mathbb{Y}_{r r}^{+} \neq \emptyset$. Therefore, sets ${ }_{r} \mathbb{Y}_{i} \neq \emptyset, i \in{ }_{r} \mathbb{X}$.

Thus, conditions A (a) and (b) assumed to hold for the Markov chain $\eta_{n}^{(\varepsilon)}$, imply that these conditions also hold for the Markov chain ${ }_{r} \eta_{n}^{(\varepsilon)}$, with sets ${ }_{r} \mathbb{Y}_{i}, i \in{ }_{r} \mathbb{X}$ replacing sets $\mathbb{Y}_{i}, i \in \mathbb{X}$.

Also, let $i, j \in{ }_{r} \mathbb{X}$ and $i=l_{0}, l_{1}, \ldots, l_{n_{i j}}=j$ be a chain of states such that $l_{1} \in \mathbb{Y}_{l_{0}}, \ldots, l_{n} \in \mathbb{Y}_{l_{n_{i j}-1}}$. As was remarked above, we can always to assume that states $l_{1}, \ldots, l_{n_{i j}-1}$ are different and that $l_{1}, \ldots, l_{n_{i j}-1} \neq i, j$. This implies that either $l_{1}, \ldots, l_{n_{i j}-1} \neq r$ or there exist at most one $1 \leq k \leq$ $n_{i j}-1$ such that $i_{k}=r$. In the first case, $l_{1} \in{ }_{r} \mathbb{Y}_{l_{0}}, \ldots, l_{n_{i j}} \in{ }_{r} \mathbb{Y}_{l_{n_{i j}-1}}$. In the second case, $l_{1} \in{ }_{r} \mathbb{Y}_{l_{0}}, \ldots, l_{k-1} \in{ }_{r} \mathbb{Y}_{l_{k-2}}, l_{k-1} \in{ }_{r} \mathbb{Y}_{l_{k+1}}, \ldots, l_{n_{i j}} \in{ }_{r} \mathbb{Y}_{l_{n_{i j}-1}}$.

Thus, condition A (c) assumed to hold for the Markov chain $\eta_{n}^{(\varepsilon)}$, imply that this condition also holds for the Markov chain ${ }_{r} \eta_{n}^{(\varepsilon)}$. 
Let us define distribution functions,

$$
F_{i}^{(\varepsilon)}(t)=\sum_{j \in \mathbb{X}} Q_{i j}^{(\varepsilon)}(t), t \geq 0, i, j \in \mathbb{X} .
$$

and

$$
F_{i j}^{(\varepsilon)}(t)=\left\{\begin{array}{cc}
Q_{i j}^{(\varepsilon)}(t) / p_{i j}(\varepsilon) & \text { for } t \geq 0 \text { if } p_{i j}(\varepsilon)>0 \\
F_{i}^{(\varepsilon)}(t) & \text { for } t \geq 0 \text { if } p_{i j}(\varepsilon)=0
\end{array}\right.
$$

Obviously,

$$
\tilde{e}_{i j}(\varepsilon)=\int_{0}^{\infty} t F_{i j}^{(\varepsilon)}(d t)=\left\{\begin{array}{cc}
e_{i j}(\varepsilon) / p_{i j}(\varepsilon) & \text { if } p_{i j}(\varepsilon)>0 \\
e_{i}(\varepsilon) & \text { if } p_{i j}(\varepsilon)=0
\end{array}\right.
$$

and

$$
e_{i}(\varepsilon)=\int_{0}^{\infty} t F_{i}^{(\varepsilon)}(d t), i \in \mathbb{X}
$$

Also, let us introduce expectations,

$$
{ }_{r} e_{i j}(\varepsilon)=\int_{0}^{\infty} t_{r} Q_{i j}^{(\varepsilon)}(d t), i, j \in{ }_{r} \mathbb{X} .
$$

Relation (85) directly implies the following formula for expectations ${ }_{r} e_{i j}(\varepsilon)$, $i, j \in r_{r} \mathbb{X}$

$$
\begin{aligned}
{ }_{r} e_{i j}(\varepsilon)= & \tilde{e}_{i j}(\varepsilon) p_{i j}(\varepsilon)+\sum_{n=0}^{\infty}\left(\tilde{e}_{i r}(\varepsilon)+n \tilde{e}_{r r}(\varepsilon)+\tilde{e}_{r j}(\varepsilon)\right) p_{i r}(\varepsilon) p_{r r}(\varepsilon)^{n} p_{r j}(\varepsilon) \\
= & e_{i j}(\varepsilon)+e_{i r}(\varepsilon) \frac{p_{r j}(\varepsilon)}{1-p_{r r}(\varepsilon)} \\
& +e_{r r}(\varepsilon) \frac{p_{i r}(\varepsilon)}{1-p_{r r}(\varepsilon)} \frac{p_{r j}(\varepsilon)}{1-p_{r r}(\varepsilon)}+e_{r j}(\varepsilon) \frac{p_{i r}(\varepsilon)}{1-p_{r r}(\varepsilon)}
\end{aligned}
$$

Relation (96) implies that conditions $\mathbf{D}$ and $\mathbf{E}$, assumed to hold for the semi-Markov process $\eta^{(\varepsilon)}(t)$, imply that these conditions also hold for the semi-Markov process ${ }_{r} \eta^{(\varepsilon)}(t)$.

5.2. Hitting times for reduced semi-Markov processes. The first hitting times to a state $j \neq r$ are connected for Markov chains $\eta_{n}^{(\varepsilon)}$ and ${ }_{r} \eta_{n}^{(\varepsilon)}$ by the following relation,

$$
\begin{aligned}
\nu_{j}^{(\varepsilon)} & =\min \left(n \geq 1: \eta_{n}^{(\varepsilon)}=j\right) \\
& =\min \left({ }_{r} \xi_{n}^{(\varepsilon)} \geq 1:{ }_{r} \eta_{n}^{(\varepsilon)}=j\right)={ }_{r} \xi_{r}^{(\varepsilon)} \nu_{j}^{(\varepsilon)}
\end{aligned}
$$


where

$$
{ }_{r} \nu_{j}^{(\varepsilon)}=\min \left(n \geq 1:{ }_{r} \eta_{n}^{(\varepsilon)}=j\right) .
$$

Relations (97) and (98) imply that the following relation hold for the first hitting times to a state $j \neq r$ for the semi-Markov processes $\eta^{(\varepsilon)}(t)$ and ${ }_{r} \eta^{(\varepsilon)}(t)$,

$$
\begin{aligned}
\tau_{j}^{(\varepsilon)} & =\sum_{n=1}^{\nu_{j}^{(\varepsilon)}} \kappa_{n}^{(\varepsilon)}=\sum_{n=1}^{{ }_{r}^{\xi^{(\varepsilon)} \nu_{j}^{(\varepsilon)}}{ }_{n}^{(\varepsilon)}} \kappa_{n}^{(\varepsilon)} \\
= & \sum_{n=1}{ }_{r}^{(\varepsilon)} \kappa_{n}^{(\varepsilon)}={ }_{r} \tau_{j}^{(\varepsilon)}
\end{aligned}
$$

Let us summarize the above remarks in the following theorem, which play the key role in what follows.

Theorem 1. Let conditions $\mathbf{A}, \mathbf{D}$ and $\mathbf{E}$ hold and the initial distribution satisfies the assumption, $p_{r}^{(\varepsilon)}=0$, for every $\varepsilon \in\left(0, \varepsilon_{0}\right]$. Then, for any state $j \neq r$, the first hitting times $\tau_{j}^{(\varepsilon)}$ and ${ }_{r} \tau_{j}^{(\varepsilon)}$ to the state $j$, respectively, for semi-Markov processes $\eta^{(\varepsilon)}(t)$ and ${ }_{r} \eta^{(\varepsilon)}(t)$, coincide.

\section{Asymptotic expansions for transition characteristics of per-} turbed semi-Markov processes with reduced phase spaces

In this section, we present algorithms for re-calculation of asymptotic expansions for perturbed semi-Markov processes with reduced phase spaces.

6.1 Asymptotic expansions for non-absorption probabilities. As was mentioned above, condition $\mathbf{A}$ implies that the non-absorption probability $\bar{p}_{i i}(\varepsilon)=1-p_{i i}(\varepsilon)>0, i \in \mathbb{X}, \varepsilon \in\left(0, \varepsilon_{0}\right]$.

Let us introduce the set,

$$
\mathbb{Y}=\left\{i \in \mathbb{X}: i \in \mathbb{Y}_{i}\right\}
$$

Algorithm 1. This is an algorithm for constructing asymptotic expansions for non-absorption probabilities $\bar{p}_{i i}(\varepsilon), i \in \mathbb{X}$.

Case 1: $i \in \mathbb{Y}$. 
Let us use the following relation, which holds, for every $i \in \mathbb{Y}$ and $\varepsilon \in$ $\left(0, \varepsilon_{0}\right]$

$$
\bar{p}_{i i}(\varepsilon)=1-p_{i i}(\varepsilon)=\sum_{j \in \mathbb{Y}_{i i}^{+}} p_{i j}(\varepsilon)
$$

1.1. To construct the $\left(h_{i}^{\prime}, k_{i}^{\prime}\right)$-expansion for the non-absorption probability $\bar{p}_{i i}(\varepsilon)=1-p_{i i}(\varepsilon)$ by applying the propositions (i) (the multiplication by a constant rule) and (ii) (the summation rule) of Lemma 3 to the $\left(l_{i i}^{-}, l_{i i}^{+}\right)$expansion for transition probability $p_{i i}(\varepsilon)$ given in condition $\mathbf{B}$ (first, this expansion is multiplied by constant -1 and, second, is summated with constant 1 represented as $\left(0, l_{i i}^{+}\right)$-expansion given in relation (58) $)$. In this case, parameters $h_{i}^{\prime}=0, k_{i}^{\prime}=l_{i i}^{+}$.

1.2. To construct the $\left(h_{i}^{\prime \prime}, k_{i}^{\prime \prime}\right)$-expansion for the non-absorption probability $\bar{p}_{i i}(\varepsilon)=\sum_{j \in \mathbb{Y}_{i i}^{+}} p_{i j}(\varepsilon)$ using the corresponding asymptotic expansions for transition probabilities $p_{i j}(\varepsilon), j \in \mathbb{Y}_{i i}^{+}$given in condition $\mathbf{B}$, and the proposition (i) (the multiple summation rule) of Lemma 5. In this case, parameters $h_{i}^{\prime \prime}=\min _{j \in \mathbb{Y}_{i i}^{+}} l_{i j}^{-}, k_{i}^{\prime \prime}=\min _{j \in \mathbb{Y}_{i i}^{+}} l_{i j}^{+}$. This asymptotic expansion is pivotal.

1.3. To construct the $\left(\bar{h}_{i}, \bar{k}_{i}\right)$-expansion for the non-absorption probability $\bar{p}_{i i}(\varepsilon)$ using relation (101), and propositions (i) - (iv) of Lemma 1. In this case, parameters $\bar{h}_{i}=0 \vee h_{i}^{\prime \prime}=h_{i}^{\prime \prime}, \bar{k}_{i}=k_{i}^{\prime} \vee k_{i}^{\prime \prime}=l_{i i}^{+} \vee \min _{j \in \mathbb{Y}_{i i}^{+}} l_{i j}^{+}$. This asymptotic expansion is pivotal.

It should be noted that $\left(l_{i i}^{-}, l_{i i}^{+}\right)$-expansion for the transition probability $p_{i i}(\varepsilon)$ given in condition $\mathbf{B}$ and $\left(h_{i}^{\prime \prime}, k_{i}^{\prime \prime}\right)$-expansion for function $\bar{p}_{i i}(\varepsilon)=$ $\sum_{j \in \mathbb{Y}_{i i}^{+}} p_{i j}(\varepsilon)$ given in Step 1.2, satisfy, for every $i \in \mathbb{X}$, additional conditions given in Remark 1, respectively, for set $\mathbb{Z}=\{i\}$ and set $\mathbb{Z}=\mathbb{Y}_{i i}^{+}$.

Case 2: $i \in \overline{\mathbb{Y}}$.

1.4. In this case, the non-absorption probability $\bar{p}_{i i}(\varepsilon) \equiv 1$. If necessary, it can be represented in the form of $(0, n)$-expansion given by relation (58), for any $n=0,1, \ldots$.

The above remarks can be summarized in the following theorem.

Lemma 10. Let conditions $\mathbf{A}, \mathbf{B}$ and $\mathbf{C}$ hold. Then, the asymptotic expansions for the non-absorption probabilities $\bar{p}_{i i}(\varepsilon), i \in \mathbb{X}$ are given in Algorithm 1.

Algorithm 2. This is an algorithm for computing upper bounds for remainders of asymptotic expansions for non-absorption probabilities $\bar{p}_{i i}(\varepsilon)$, $i \in \mathbb{X}$. 
Case 1: $i \in \mathbb{Y}$.

2.1. To construct $\left(h_{i}^{\prime}, k_{i}^{\prime}, \delta_{i}^{\prime}, G_{i}^{\prime}, \varepsilon_{i}^{\prime}\right)$-expansion for the non-absorption probability $\bar{p}_{i i}(\varepsilon)=1-p_{i i}(\varepsilon)$ by applying the propositions (i) (the multiplication by a constant rule) and (ii (the summation rule) of Lemma 4 to the $\left(l_{i i}^{-}, l_{i i}^{+}, \delta_{i i}, G_{i i}, \varepsilon_{i i}\right)$-expansion for the transition probability $p_{i i}(\varepsilon)$ given in condition $\mathbf{B}^{\prime}$ and (first, this expansion is multiplied by constant -1 and, second, is summated with constant 1 represented as $\left(0, l_{i i}^{+}, 1, G, \varepsilon_{0}\right)$-expansion given in relation (58) ), third, constant $G$ can be replaced by 0 , since it can be taken an arbitrary small. In this case, parameters $\delta_{i}^{\prime}=\delta_{i i}, G_{i}^{\prime}=G_{i i}, \varepsilon_{i}^{\prime}=\varepsilon_{i i}$.

2.2. To construct the $\left(h_{i}^{\prime \prime}, k_{i}^{\prime \prime}, \delta_{i}^{\prime \prime}, G_{i}^{\prime \prime}, \varepsilon_{i}^{\prime \prime}\right)$-expansion for the non-absorption probability $\bar{p}_{i i}(\varepsilon)=\sum_{j \in \mathbb{Y}_{i i}^{+}} p_{i j}(\varepsilon)$ using the $\left(l_{i j}^{-}, l_{i j}^{+}, \delta_{i j}, G_{i j}, \varepsilon_{i j}\right)$-expansions for transition probabilities $p_{i j}(\varepsilon), j \in \mathbb{Y}_{i i}^{+}$given in condition $\mathbf{B}^{\prime}$, and the proposition (i) (the multiple summation rule) of Lemma 6 . In this case, parameters $\delta_{i}^{\prime \prime}, G_{i}^{\prime \prime}, \varepsilon_{i}^{\prime \prime}$ are given by the corresponding variant of relation (24).

2.3. To construct the $\left(\bar{h}_{i}, \bar{k}_{i}, \bar{\delta}_{i}, \bar{G}_{i}, \bar{\varepsilon}_{i}\right)$-expansion for the non-absorption probability $\bar{p}_{i i}(\varepsilon)$ using relation (101), and proposition (i) of Lemma 2. In this case, parameters $\bar{\delta}_{i}, \bar{G}_{i}, \bar{\varepsilon}_{i}$ are given by the corresponding variant of relation (4).

Case 2: $i \in \overline{\mathbb{Y}}$.

2.4. In this case, the non-absorption probability $\bar{p}_{i i}(\varepsilon) \equiv 1$. If necessary, it can be represented in the form of $\left(0, n, 1, G, \varepsilon_{0}\right)$-expansion given by relation(58), for any $0<G<\infty$ and $n=0,1, \ldots$.

The above remarks can be summarized in the following theorem.

Lemma 11. Let conditions $\mathbf{A}, \mathbf{B}^{\prime}$ and $\mathbf{C}$ hold. Then, the asymptotic expansions for the non-absorption probabilities $\bar{p}_{i i}(\varepsilon), i \in \mathbb{X}$ with explicit upper bounds for remainders are given in Algorithm 2.

6.2. Asymptotic expansions for transition probabilities of reduced embedded Markov chains. Relation (87) can be re-written in the following form more convenient for constructing asymptotic expansions for probabilities ${ }_{r} p_{i j}(\varepsilon), i, j \in{ }_{r} \mathbb{X}$,

$$
{ }_{r} p_{i j}(\varepsilon)=\left\{\begin{array}{cl}
p_{i j}(\varepsilon)+p_{i r}(\varepsilon) \frac{p_{r j}(\varepsilon)}{1-p_{r r}(\varepsilon)} & \text { if } j \in \mathbb{Y}_{i r}^{+} \cap \mathbb{Y}_{i r}^{-}, \\
p_{i j}(\varepsilon) & \text { if } j \in \mathbb{Y}_{i r}^{+} \cap \overline{\mathbb{Y}}_{i r}^{-}, \\
p_{i r}(\varepsilon) \frac{p_{r j}(\varepsilon)}{1-p_{r r}(\varepsilon)} & \text { if } j \in \overline{\mathbb{Y}}_{i r}^{+} \cap \mathbb{Y}_{i r}^{-}, \\
0 & \text { if } j \in \overline{\mathbb{Y}}_{i r}^{+} \cap \overline{\mathbb{Y}}_{i r}^{-}={ }_{r} \overline{\mathbb{Y}}_{i} .
\end{array}\right.
$$


Algorithm 3. This is an algorithm for constructing asymptotic expansions for transition probabilities ${ }_{r} p_{i j}(\varepsilon), i, j \in{ }_{r} \mathbb{X}$.

Case 1: $r \in \mathbb{Y}$.

3.1. To construct $\left(\tilde{h}_{r j}, \tilde{k}_{r j}\right)$-expansions for conditional probabilities $\tilde{p}_{r j}(\varepsilon)$ $=\frac{p_{r j}(\varepsilon)}{1-p_{r r}(\varepsilon)}, j \in \mathbb{Y}_{r r}^{+}$, using the $\left(l_{r j}^{-}, l_{r j}^{+}\right)$-expansions for transition probabilities $p_{r j}(\varepsilon)$ given in condition $\mathbf{B}$, the $\left(\bar{h}_{r}, \bar{k}_{r}\right)$-expansion for the non-absorption probability $\bar{p}_{r r}(\varepsilon)=1-p_{r r}(\varepsilon)$ given in Algorithm 1, and the proposition (v) (the division rule) of Lemma 3. In this case, parameters $\tilde{h}_{r j}=l_{r j}^{-}-\bar{h}_{r}, \tilde{k}_{r j}=$ $\left(l_{r j}^{+}-\bar{h}_{r}\right) \wedge\left(l_{r j}^{-}+\bar{k}_{r}-2 \bar{h}_{r}\right), j \in \mathbb{Y}_{r r}^{+}$. These asymptotic expansions are pivotal.

3.2. To construct $\left(\grave{h}_{i r j}, \grave{k}_{i r j}\right)$-expansions for products $\grave{p}_{i r j}(\varepsilon)=p_{i r}(\varepsilon) \tilde{p}_{r j}(\varepsilon)$ $=p_{i r}(\varepsilon) \cdot \frac{p_{r j}(\varepsilon)}{1-p_{r r}(\varepsilon)}, j \in \mathbb{Y}_{i r}^{-}, i \in{ }_{r} \mathbb{X}$, using the $\left(l_{i r}^{-}, l_{i r}^{+}\right)$-expansions for transition probabilities $p_{i r}(\varepsilon)$ given in condition $\mathbf{B}$, the $\left(\tilde{h}_{r j}, \tilde{k}_{r j}\right)$-expansions for conditional probabilities $\tilde{p}_{r j}(\varepsilon)$ given in the above Step 3.1 and the proposition (iii) (the multiplication rule) of Lemma 3. In this case, parameters $\grave{h}_{i r j}=l_{i r}^{-}+\tilde{h}_{r j}, \grave{k}_{i r j}=\left(l_{i r}^{-}+\tilde{k}_{r j}\right) \wedge\left(l_{i r}^{+}+\tilde{h}_{r j}\right), \quad j \in \mathbb{Y}_{i r}^{-}, i \in{ }_{r} \mathbb{X}$. These asymptotic expansions are pivotal.

3.3. To construct $\left(\hat{h}_{i r j}, \hat{k}_{i r j}\right)$-expansions for sums $\dot{p}_{i r j}(\varepsilon)=p_{i j}(\varepsilon)+$ $\grave{p}_{i r j}(\varepsilon)=p_{i j}(\varepsilon)+p_{i r}(\varepsilon) \cdot \frac{p_{r j}(\varepsilon)}{1-p_{r r}(\varepsilon)}, j \in \mathbb{Y}_{i r}^{+} \cap \mathbb{Y}_{i r}^{-}, i \in{ }_{r} \mathbb{X}$, using the $\left(l_{i j}^{-}, l_{i j}^{+}\right)-$ expansions for transition probabilities $p_{i j}(\varepsilon)$ given in condition $\mathbf{B}$, the $\left(\grave{h}_{i r j}\right.$, $\grave{k}_{i r j}$ )-expansions for quantities $\grave{p}_{i r j}(\varepsilon)$ given in the above Step 3.2 and the proposition (ii) (the summation rule) of Lemma 3. In this case, parameters $\hat{h}_{i r j}=l_{i j}^{-} \wedge \grave{h}_{i r j}, \hat{k}_{i r j}=l_{i j}^{+} \wedge \grave{k}_{i r j}, j \in \mathbb{Y}_{i r}^{-}, i \in{ }_{r} \mathbb{X}$. These asymptotic expansions are pivotal.

3.4. To construct $\left({ }_{r} l_{i j}^{-},{ }_{r} l_{i j}^{+}\right)$-expansions for transition probabilities ${ }_{r} p_{i j}(\varepsilon)$ $=\sum_{l=r r_{i j}}^{r} l_{i j}^{+}{ }_{i j}[l] \varepsilon^{l}+o\left(\varepsilon^{r} l_{i j}^{+}\right), i, j \in{ }_{r} \mathbb{X}$, using the $\left(l_{i j}^{-}, l_{i j}^{+}\right)$-expansions for transition probabilities $p_{i j}(\varepsilon)$ given in condition $\mathbf{B}$, the $\left(\grave{h}_{i r j}, \grave{k}_{i r j}\right)$-expansions for quantities $\grave{p}_{i r j}(\varepsilon)$ and $\left(\hat{h}_{i r j}, \dot{k}_{i r j}\right)$-expansions for quantities $\dot{p}_{i r j}(\varepsilon)$ given, respectively, in the above Steps 3.2 and 3.3, and the corresponding variants of formulas for transition probabilities ${ }_{r} p_{i j}(\varepsilon)$ given in relation (102). In this case, parameters ${ }_{r} l_{i j}^{-}=\hat{h}_{i r j}{ }_{r} l_{i j}^{+}=\dot{k}_{i r j}$ if $j \in \mathbb{Y}_{i r}^{+} \cap \mathbb{Y}_{i r}^{-}, i \in{ }_{r} \mathbb{X}$, or ${ }_{r} l_{i j}^{-}=l_{i j}^{-},{ }_{r} l_{i j}^{+}=l_{i j}^{+}$if $j \in \mathbb{Y}_{i r}^{+} \cap \overline{\mathbb{Y}}_{i r}^{-}, i \in{ }_{r} \mathbb{X}$, or ${ }_{r} l_{i j}^{-}=\grave{h}_{i r j}{ }_{r} l_{i j}^{+}=\grave{k}_{i r j}$ if $j \in \overline{\mathbb{Y}}_{i r}^{+} \cap \mathbb{Y}_{i r}^{-}, i \in{ }_{r} \mathbb{X}$. These asymptotic expansions are pivotal.

Case 2: $r \in \overline{\mathbb{Y}}$.

3.5. The corresponding algorithm is a particular case of the algorithm 
given in Steps 3.1 - 3.4. In this case the non-absorption probability $\bar{p}_{r r}(\varepsilon)=$ $1-p_{r r}(\varepsilon) \equiv 1$ and, thus, conditional probabilities $\tilde{p}_{r j}(\varepsilon)=\frac{p_{r j}(\varepsilon)}{1-p_{r r}(\varepsilon)}=p_{r j}(\varepsilon)$, $j \in \mathbb{Y}_{r r}^{+}$. This permits one replace the $\left(\tilde{h}_{r j}, \tilde{k}_{r j}\right)$-expansions for conditional probabilities $\tilde{p}_{r j}(\varepsilon)$ by the $\left(l_{r j}^{-}, l_{r j}^{+}\right)$-expansions for transition probabilities $p_{r j}(\varepsilon)$. This is the only change in the algorithm for construction of asymptotic expansions for transition probabilities ${ }_{r} p_{i j}(\varepsilon), i, j \in{ }_{r} \mathbb{X}$ given in Steps $3.1-3.4$, which is required.

The above remarks can be summarized in the following theorem.

Theorem 2. Conditions A, B and $\mathbf{C}$ assumed to hold for the Markov chain $\eta_{n}^{(\varepsilon)}$, also hold for the reduced Markov chain ${ }_{r} \eta_{n}^{(\varepsilon)}$, for every $r \in \mathbb{X}$. The asymptotic expansions penetrating condition $\mathbf{B}$ are given for transition probabilities ${ }_{r} p_{i j}(\varepsilon), j \in{ }_{r} \mathbb{Y}_{i}, i \in{ }_{r} \mathbb{X}, r \in \mathbb{X}$ in Algorithm 3.

Algorithm 4. This is an algorithm for computing upper bounds for remainders in asymptotic expansions for transition probabilities ${ }_{r} p_{i j}(\varepsilon), i, j \in$ ${ }_{r} \mathbb{X}$.

4.1. To construct $\left(\tilde{h}_{r j}, \tilde{k}_{r j}, \tilde{\delta}_{r j}, \tilde{G}_{r j}, \tilde{\varepsilon}_{r j}\right)$-expansions for conditional probabilities $\tilde{p}_{r j}(\varepsilon)=\frac{p_{r j}(\varepsilon)}{1-p_{r r}(\varepsilon)}, j \in \mathbb{Y}_{r r}^{+}$using the $\left(l_{r j}^{-}, l_{r j}^{+}, \delta_{r j}, G_{r j}, \varepsilon_{r j}\right)$-expansions for transition probabilities $p_{r j}(\varepsilon)$ given in condition $\mathbf{B}^{\prime}$, the $\left(\bar{h}_{r}, \bar{k}_{r}, \bar{\delta}_{r j}, \bar{G}_{r j}, \bar{\varepsilon}_{r j}\right)$ expansion for the non-absorption probability $\bar{p}_{r r}(\varepsilon)=1-p_{r r}(\varepsilon)$ given in Algorithm 2, and the proposition (v) (the division rule) of Lemma 4. In this case, parameters $\tilde{\delta}_{r j}, \tilde{G}_{r j}, \tilde{\varepsilon}_{r j}, j \in \mathbb{Y}_{r r}^{+}$are given by the corresponding variants of relation (17).

4.2. To construct $\left(\grave{h}_{i r j}, \grave{k}_{i r j}, \grave{\delta}_{i r j}, \grave{G}_{i r j}, \grave{\varepsilon}_{i r j}\right)$-expansions for products $\grave{p}_{i r j}(\varepsilon)$ $=p_{i r}(\varepsilon) \tilde{p}_{r j}(\varepsilon)=p_{i r}(\varepsilon) \cdot \frac{p_{r j}(\varepsilon)}{1-p_{r r}(\varepsilon)}, j \in \mathbb{Y}_{i r}^{-}, i \in{ }_{r} \mathbb{X}$, using the $\left(l_{i r}^{-}, l_{i r}^{+}, \delta_{i r}, G_{i r}, \varepsilon_{i r}\right)-$ expansions for transition probabilities $p_{i r}(\varepsilon)$ given in condition $\mathbf{B}^{\prime}$, the $\left(\tilde{h}_{r j}, \tilde{k}_{r j}\right.$, $\tilde{\delta}_{r j}, \tilde{G}_{r j}, \tilde{\varepsilon}_{r j}$ )-expansions for conditional probabilities $\tilde{p}_{r j}(\varepsilon)$ given in the above Step 4.1 and the proposition (iii) (the multiplication rule) of Lemma 4 . In this case, parameters $\grave{\delta}_{i r j}, \grave{G}_{i r j}, \grave{\varepsilon}_{i r j}, j \in \mathbb{Y}_{i r}^{-}, i \in{ }_{r} \mathbb{X}$ are given by the corresponding variants of relation (15).

4.3. To construct $\left(\hat{h}_{i r j}, \hat{k}_{i r j}, \hat{\delta}_{i r j}, \dot{G}_{i r j}, \dot{\varepsilon}_{i r j}\right)$-expansions for sums $\dot{p}_{i r j}(\varepsilon)=$ $p_{i j}(\varepsilon)+\grave{p}_{i r j}(\varepsilon)=p_{i j}(\varepsilon)+p_{i r}(\varepsilon) \cdot \frac{p_{r j}(\varepsilon)}{1-p_{r r}(\varepsilon)}, j \in \mathbb{Y}_{i r}^{+} \cap \mathbb{Y}_{i r}^{-}, \quad i \in{ }_{r} \mathbb{X}$, using the $\left(l_{i j}^{-}, l_{i j}^{+}, \delta_{i j}, G_{i j}, \varepsilon_{i j}\right)$-expansions for transition probabilities $p_{i j}(\varepsilon)$ given in condition $\mathbf{B}^{\prime}$, the $\left(\grave{h}_{i r j}, \grave{k}_{i r j}, \grave{\delta}_{i r j}, \grave{G}_{i r j}, \grave{\varepsilon}_{i r j}\right)$-expansions for quantities $\grave{p}_{i r j}(\varepsilon)$ given in the above Step 4.2 and the proposition (ii) (the summation rule) of Lemma 4. In this case, parameters $\delta_{i r j}, G_{i r j}, \dot{\varepsilon}_{i r j}, j \in \mathbb{Y}_{i r}^{-}, i \in{ }_{r} \mathbb{X}$ are given 
by the corresponding variants of relation (14).

4.4. To construct $\left({ }_{r} l_{i j}^{-},{ }_{r} l_{i j}^{+},{ }_{r} \delta_{i j},{ }_{r} G_{i j},{ }_{r} \varepsilon_{i j}\right)$-expansions for transition probabilities ${ }_{r} p_{i j}(\varepsilon)=\sum_{l={ }_{r} l_{i j}^{-}}^{{ }_{r} l_{r j}^{+}} a_{i j}[l] \varepsilon^{l}+o\left(\varepsilon^{r} l_{i j}^{+}\right), i, j \in{ }_{r} \mathbb{X}$ using the $\left(l_{i j}^{-}, l_{i j}^{+}, \delta_{i j}, G_{i j}\right.$, $\varepsilon_{i j}$ )-expansions for transition probabilities $p_{i j}(\varepsilon)$ given in condition $\mathbf{B}^{\prime}$, the $\left(\grave{h}_{i r j}, \grave{k}_{i r j}, \grave{\delta}_{i r j}, \grave{G}_{i r j}, \grave{\varepsilon}_{i r j}\right)$-expansions for quantities $\grave{p}_{i r j}(\varepsilon)$ and $\left(\grave{h}_{i r j}, \dot{k}_{i r j}, \dot{\delta}_{i r j}\right.$, $\left.\dot{G}_{i r j}, \dot{\varepsilon}_{i r j}\right)$-expansions for quantities $\dot{p}_{i r j}(\varepsilon)$ given, respectively, in the above Steps 4.2 and 4.3 , and the corresponding variants of formulas for transition probabilities ${ }_{r} p_{i j}(\varepsilon)$ given in relation (102). In this case, parameters ${ }_{r} \delta_{i j}=\dot{\delta}_{i r j,}{ }_{r} G_{i j}=\dot{G}_{i r j},{ }_{r} \varepsilon_{i j}=\dot{\varepsilon}_{i r j}$ if $j \in \mathbb{Y}_{i r}^{+} \cap \mathbb{Y}_{i r}^{-}, i \in{ }_{r} \mathbb{X}$, or ${ }_{r} \delta_{i j}=\delta_{i j},{ }_{r} G_{i j}=G_{i j},{ }_{r} \varepsilon_{i j}=\varepsilon_{i j}$ if $j \in \mathbb{Y}_{i r}^{+} \cap \overline{\mathbb{Y}}_{i r}^{-}, i \in{ }_{r} \mathbb{X}$, or ${ }_{r} \delta_{i j}=\grave{\delta}_{i r j},{ }_{r} G_{i j}=$ $\grave{G}_{i r j},{ }_{r} \varepsilon_{i j}=\grave{\varepsilon}_{i r j}$ if $j \in \overline{\mathbb{Y}}_{i r}^{+} \cap \mathbb{Y}_{i r}^{-}, i \in{ }_{r} \mathbb{X}$.

Case 2: $r \in \overline{\mathbb{Y}}$.

4.5. The corresponding algorithm is a particular case of the algorithm given in Steps $4.1-4.4$. In this case the non-absorption probability $\bar{p}_{r r}(\varepsilon)=$ $1-p_{r r}(\varepsilon) \equiv 1$ and, thus, conditional probabilities $\tilde{p}_{r j}(\varepsilon)=\frac{p_{r j}(\varepsilon)}{1-p_{r r}(\varepsilon)}=p_{r j}(\varepsilon), i \in$ $\mathbb{Y}_{r r}^{+}$. This permits one replace the $\left(\tilde{h}_{r j}, \tilde{k}_{r j}, \tilde{\delta}_{r j}, \tilde{G}_{r j}, \tilde{\varepsilon}_{r j}\right)$-expansions for conditional probabilities $\tilde{p}_{r j}(\varepsilon)$ by the $\left(l_{r j}^{-}, l_{r j}^{+}, \delta_{r j}, G_{r j}, \varepsilon_{r j}\right)$-expansions for transition probabilities $p_{r j}(\varepsilon)$. This is the only change in the algorithm for construction of asymptotic expansions for transition probabilities ${ }_{r} p_{i j}(\varepsilon), i, j \in$ $r \mathbb{X}$ given in Steps $4.1-4.4$, which is required.

The above remarks can be summarized in the following theorem.

Theorem 3. Conditions $\mathbf{A}, \mathbf{B}^{\prime}$ and $\mathbf{C}$ assumed to hold for the Markov chain $\eta_{n}^{(\varepsilon)}$, also hold for the reduced Markov chain ${ }_{r} \eta_{n}^{(\varepsilon)}$, for every $r \in \mathbb{X}$. The upper bounds for remainders in asymptotic expansions penetrating condition $\mathbf{B}^{\prime}$ are given for transition probabilities ${ }_{r} p_{i j}(\varepsilon), j \in{ }_{r} \mathbb{Y}_{i}, i \in{ }_{r} \mathbb{X}, r \in \mathbb{X}$ in Algorithm 4.

\subsection{Asymptotic expansions for expectations of sojourn times} for reduced semi-Markov processes. Relation (96) can be re-written in the following form more convenient for constructing the corresponding 
asymptotic expansions probabilities ${ }_{r} e_{i j}(\varepsilon), i, j \in{ }_{r} \mathbb{X}$,

$$
{ }_{r} e_{i j}(\varepsilon)= \begin{cases}e_{i j}(\varepsilon)+e_{i r}(\varepsilon) \frac{p_{r j}(\varepsilon)}{1-p_{r r}(\varepsilon)} & \\ +e_{r r}(\varepsilon) \frac{p_{i r}(\varepsilon)}{1-p_{r r}(\varepsilon)} \frac{p_{r j}(\varepsilon)}{1-p_{r r}(\varepsilon)} & \\ +e_{r j}(\varepsilon) \frac{p_{i r}(\varepsilon)}{1-p_{r r}(\varepsilon)} & \text { if } j \in \mathbb{Y}_{i r}^{+} \cap \mathbb{Y}_{i r}^{-}, \\ e_{i j}(\varepsilon) & \text { if } j \in \mathbb{Y}_{i r}^{+} \cap \overline{\mathbb{Y}}_{i r}^{-}, \\ e_{i r}(\varepsilon) \frac{p_{r j}(\varepsilon)}{1-p_{r r}(\varepsilon)} & \\ +e_{r r}(\varepsilon) \frac{p_{i r}(\varepsilon)}{1-p_{r r}(\varepsilon)} \frac{p_{r j}(\varepsilon)}{1-p_{r r}(\varepsilon)} & \text { if } j \in \overline{\mathbb{Y}}_{i r}^{+} \cap \mathbb{Y}_{i r}^{-}, \\ +e_{r j}(\varepsilon) \frac{p_{i r}(\varepsilon)}{1-p_{r r}(\varepsilon)} & \text { if } j \in \overline{\mathbb{Y}}_{i r}^{+} \cap \overline{\mathbb{Y}}_{i r}^{-} . \\ 0 & \end{cases}
$$

Algorithm 5. This is an algorithm for computing asymptotic expansions for expectations ${ }_{r} e_{i j}(\varepsilon), i, j \in{ }_{r} \mathbb{X}$.

Case 1: $r \in \mathbb{Y}$.

5.1. To construct $\left(\hat{h}_{i r}, \hat{k}_{i r}\right)$-expansions for quantities $\hat{p}_{i r}(\varepsilon)=\frac{p_{i r}(\varepsilon)}{\bar{p}_{r r}(\varepsilon)}=$ $\frac{p_{i r}(\varepsilon)}{1-p_{r r}(\varepsilon)}, i \in{ }_{r} \mathbb{X}$, using the $\left(l_{i r}^{-}, l_{i r}^{+}\right)$-expansions for transition probabilities $p_{i r}(\varepsilon)$ given in condition $\mathbf{B}$, the $\left(\bar{h}_{r}, \bar{k}_{r}\right)$-expansion for the non-absorption probability $\bar{p}_{r}(\varepsilon)=1-p_{r r}(\varepsilon)$ given in Algorithm 1, and proposition (v) (the division rule) of Lemma 3. In this case, parameters $\hat{h}_{i r}=h_{i r}-\bar{h}_{r}, \hat{k}_{i r}=$ $\left(h_{i r}+\bar{k}_{r}-2 \bar{h}_{r}\right) \wedge\left(k_{i r}-\bar{h}_{r}\right), i \in{ }_{r} \mathbb{X}$. These asymptotic expansions are pivotal.

5.2. To construct $\left(\check{h}_{i r j}, \breve{k}_{i r j}\right)$-expansions for products $\check{p}_{i r j}(\varepsilon)=\hat{p}_{i r}(\varepsilon) \tilde{p}_{r j}(\varepsilon)$ $=\frac{p_{i r}(\varepsilon)}{1-p_{r r}(\varepsilon)} \frac{p_{r j}(\varepsilon)}{1-p_{r r}(\varepsilon)}, j \in \mathbb{Y}_{i r}^{-}, i \in{ }_{r} \mathbb{X}$, using $\left(\hat{h}_{i r}, \hat{k}_{i r}\right)$-expansions given in the above Step 5.1 , the $\left(\tilde{h}_{r j}, \tilde{k}_{r j}\right)$-expansions for conditional probabilities $\tilde{p}_{r j}(\varepsilon)=\frac{p_{r j}(\varepsilon)}{1-p_{r r}(\varepsilon)}$ given in Step 3.1 of Algorithm 3, and the proposition (iii) (the multiplication rule) of Lemma 3. In this case, parameters $\check{h}_{i r}=$ $\hat{h}_{i r}+\tilde{h}_{r j}, \check{k}_{i r}=\left(\hat{h}_{i r}+\tilde{k}_{r j}\right) \wedge\left(\hat{k}_{i r}+\tilde{h}_{r j}\right), j \in \mathbb{Y}_{i r}^{-}, i \in{ }_{r} \mathbb{X}$. These asymptotic expansions are pivotal.

5.3. To construct $\left(\dot{\tilde{h}}_{i r j}, \dot{\tilde{k}}_{i r j}\right)$-expansions for products $\tilde{e}_{i r j}(\varepsilon)=e_{i r}(\varepsilon) \tilde{p}_{r j}(\varepsilon)$ $=e_{i r}(\varepsilon) \frac{p_{r j}(\varepsilon)}{1-p_{r r}(\varepsilon)}, j \in \mathbb{Y}_{i r}^{-}, i \in{ }_{r} \mathbb{X}$, using the $\left(m_{i r}^{-}, m_{i r}^{+}\right)$-expansions for expectations $e_{i r}(\varepsilon)$ given in condition $\mathbf{F}$, the $\left(\tilde{h}_{r j}, \tilde{k}_{r j}\right)$-expansions for conditional probabilities $\tilde{p}_{r j}(\varepsilon)=\frac{p_{r j}(\varepsilon)}{1-p_{r r}(\varepsilon)}$ given in Step 3.1 of Algorithm 3, and the proposition (iii) (the multiplication rule) of Lemma 3. In this case, parameters 
$\dot{\tilde{h}}_{i r j}=m_{i r}^{-}+\tilde{h}_{r j}, \dot{\tilde{k}}_{i r j}=\left(m_{i r}^{-}+\tilde{k}_{r j}\right) \wedge\left(m_{i r}^{+}+\tilde{h}_{r j}\right), j \in \mathbb{Y}_{i r}^{-}, i \in{ }_{r} \mathbb{X}$. These asymptotic expansions are pivotal.

5.4. To construct $\left(\dot{\check{h}}_{i r j}, \dot{\check{k}}_{i r j}\right)$-expansions for products $\check{e}_{i r j}(\varepsilon)=e_{r r}(\varepsilon)$ $\cdot \check{p}_{i r j}(\varepsilon)=e_{r r}(\varepsilon) \frac{p_{i r}(\varepsilon)}{1-p_{r r}(\varepsilon)} \frac{p_{r j}(\varepsilon)}{1-p_{r r}(\varepsilon)}, j \in \mathbb{Y}_{i r}^{-}, i \in{ }_{r} \mathbb{X}$, using the $\left(m_{r r}^{-}, m_{r r}^{+}\right)$expansions for expectations $e_{r r}(\varepsilon)$ given in condition $\mathbf{F}$, the $\left(\check{h}_{i r j}, \check{k}_{i r j}\right)$-expansions for quantities $\check{p}_{i r j}(\varepsilon)$ given in the above Step 5.2, and the proposition (iii) (the multiplication rule) of Lemma 3. In this case, parameters $\dot{\check{h}}_{i r j}=m_{r r}^{-}+\check{h}_{i r j}, \dot{\tilde{k}}_{i r j}=\left(m_{r r}^{-}+\check{k}_{i r j}\right) \wedge\left(m_{r r}^{+}+\check{h}_{i r j}\right), j \in \mathbb{Y}_{i r}^{-}, i \in{ }_{r} \mathbb{X}$. These asymptotic expansions are pivotal.

5.5. To construct $\left(\dot{\hat{h}}_{i r j}, \dot{\hat{k}}_{i r j}\right)$-expansions for products $\hat{e}_{i r j}(\varepsilon)=e_{r j}(\varepsilon)$ $\cdot \hat{p}_{i r}(\varepsilon)=e_{r j}(\varepsilon) \frac{p_{i r}(\varepsilon)}{1-p_{r r}(\varepsilon)}, j \in \mathbb{Y}_{i r}^{-}, i \in{ }_{r} \mathbb{X}$, using the $\left(m_{r j}^{-}, m_{r j}^{+}\right)$-expansions for expectations $e_{r j}(\varepsilon)$ given in condition $\mathbf{F}$, the $\left(\hat{h}_{i r}, \hat{k}_{i r}\right)$-expansions for quantities $\hat{p}_{i r}(\varepsilon)$ given in the above Step 5.1, and the proposition (iii) (the multiplication rule) of Lemma 3 . In this case, parameters $\dot{\hat{h}}_{i r j}=m_{r j}^{-}+\hat{h}_{i r}, \dot{\hat{k}}_{i r j}=$ $\left(m_{r j}^{-}+\hat{k}_{i r}\right) \wedge\left(m_{r j}^{+}+\hat{h}_{i r}\right), j \in \mathbb{Y}_{i r}^{-}, i \in{ }_{r} \mathbb{X}$. These asymptotic expansions are pivotal.

5.6. To construct $\left(\ddot{h}_{i r j}, \ddot{k}_{i r j}\right)$-expansions for sums $\ddot{e}_{i r j}(\varepsilon)=\tilde{e}_{i r j}(\varepsilon)+$ $\check{e}_{i r j}(\varepsilon)+\hat{e}_{i r j}(\varepsilon)=e_{i r}(\varepsilon) \frac{p_{r j}(\varepsilon)}{1-p_{r r}(\varepsilon)}+e_{r r}(\varepsilon) \frac{p_{i r}(\varepsilon)}{1-p_{r r}(\varepsilon)} \frac{p_{r j}(\varepsilon)}{1-p_{r r}(\varepsilon)}+e_{r j}(\varepsilon) \frac{p_{i r}(\varepsilon)}{1-p_{r r}(\varepsilon)}$, using the $\left(\dot{\tilde{h}}_{i r j}, \dot{\tilde{k}}_{i r j}\right)$-expansions for quantities $\tilde{e}_{i r j}(\varepsilon)$, the $\left(\dot{\tilde{h}}_{i r j}, \dot{\tilde{k}}_{i r j}\right)$-expansions for quantities $\check{e}_{i r j}(\varepsilon)$ and the $\left(\dot{\hat{h}}_{i r j}, \dot{\hat{k}}_{i r j}\right)$-expansions for quantities $\hat{e}_{i r j}(\varepsilon)$ given, respectively, in the above Steps 5.3, 5.4 and 5.5, and the proposition (i) (the summation rule) of Lemma 5. In this case, parameters $\ddot{h}_{i r j}=\dot{\tilde{h}}_{i r j} \wedge \dot{\tilde{h}}_{i r j} \wedge$ $\dot{\hat{h}}_{i r j}, \ddot{k}_{i r j}=\dot{\tilde{k}}_{i r j} \wedge \dot{\tilde{k}}_{i r j} \wedge \dot{\hat{k}}_{i r j}, j \in \mathbb{Y}_{i r}^{-}, i \in{ }_{r} \mathbb{X}$. These asymptotic expansions are pivotal.

5.7. To construct $\left(\dddot{h}_{i r j}, \dddot{k}_{i r j}\right)$-expansions for sums $\dddot{e}_{i r j}(\varepsilon)=e_{i j}(\varepsilon)+$ $\ddot{e}_{i r j}(\varepsilon)=e_{i j}(\varepsilon)+e_{i r}(\varepsilon) \frac{p_{r j}(\varepsilon)}{1-p_{r r}(\varepsilon)}+e_{r r}(\varepsilon) \frac{p_{i r}(\varepsilon)}{1-p_{r r}(\varepsilon)} \frac{p_{r j}(\varepsilon)}{1-p_{r r}(\varepsilon)}+e_{r j}(\varepsilon) \frac{p_{i r}(\varepsilon)}{1-p_{r r}(\varepsilon)}$, using $\left(m_{i j}^{-}, m_{i j}^{+}\right)$-expansions for expectations $e_{i j}(\varepsilon)$ given in condition $\mathbf{F}$, the $\left(\ddot{h}_{i r j}, \ddot{k}_{i r j}\right)$-expansions-expansions for quantities $\ddot{e}_{i r j}(\varepsilon)$ given in the above Step 5.6, and the proposition (ii) (the summation rule) of Lemma 3. In this case, parameters $\dddot{h}_{i r j}=m_{i j}^{-} \wedge \ddot{\tilde{h}}_{i r j}, \dddot{k}_{i r j}=m_{i j}^{+} \wedge \ddot{k}_{i r j}, j \in \mathbb{Y}_{i r}^{-}, i \in{ }_{r} \mathbb{X}$. These asymptotic expansions are pivotal.

5.8. To construct $\left({ }_{r} m_{i j}^{-},{ }_{r} m_{i j}^{+}\right)$-expansions for probabilities ${ }_{r} e_{i j}(\varepsilon)=$ $\sum_{l=r_{r} m_{i j}^{-}}^{r m_{i j}^{+}} b_{i j}[l] \varepsilon^{l}+o\left(\varepsilon^{r m_{i j}^{+}}\right), i, j \in{ }_{r} \mathbb{X}$ using the asymptotic expansions for 
expectations $e_{i j}(\varepsilon)$ given in condition $\mathbf{F}$, the $\left(\ddot{h}_{i r j}, \ddot{k}_{i r j}\right)$-expansions for quantities $\ddot{e}_{i r j}(\varepsilon)$ and $\left(\dddot{h}_{i r j}, \dddot{k}_{i r j}\right)$-expansions for quantities $\dddot{e}_{i r j}(\varepsilon)$ given, respectively, in Steps 5.6 and 5.7, and the corresponding variants of formulas for expectations ${ }_{r} e_{i j}(\varepsilon)$ given in relation (103). In this case, parameters ${ }_{r} m_{i j}^{-}=\dddot{h}_{i r j},{ }_{r} m_{i j}^{+}=\dddot{k}_{i r j}$ if $j \in \mathbb{Y}_{i r}^{+} \cap \mathbb{Y}_{i r}^{-}, i \in{ }_{r} \mathbb{X}$, or ${ }_{r} m_{i j}^{-}=m_{i j}^{-},{ }_{r} m_{i j}^{+}=m_{i j}^{+}$ if $j \in \mathbb{Y}_{i r}^{+} \cap \overline{\mathbb{Y}}_{i r}^{-}, i \in{ }_{r} \mathbb{X}$, or ${ }_{r} m_{i j}^{-}=\ddot{h}_{i r j},{ }_{r} m_{i j}^{+}=\ddot{k}_{i r j}$ if $j \in \overline{\mathbb{Y}}_{i r}^{+} \cap \mathbb{Y}_{i r}^{-}, i \in{ }_{r} \mathbb{X}$. These asymptotic expansions are pivotal.

Case 2: $r \in \overline{\mathbb{Y}}$.

5.9. The corresponding algorithm is a particular case of the algorithm given in Steps 5.1 - 5.8. In this case the non-absorption probability $\bar{p}_{r r}(\varepsilon)=$ $1-p_{r r}(\varepsilon) \equiv 1$ and, thus, conditional probabilities $\tilde{p}_{r j}(\varepsilon)=\frac{p_{r j}(\varepsilon)}{1-p_{r r}(\varepsilon)}=p_{r j}(\varepsilon)$, $j \in \mathbb{Y}_{r r}^{+}$and quantities $\hat{p}_{i r}(\varepsilon)=\frac{p_{i r}(\varepsilon)}{1-p_{r r}(\varepsilon)}=p_{i r}(\varepsilon), i \in{ }_{r} \mathbb{X}$. This permits one replace the $\left(\tilde{h}_{r j}, \tilde{k}_{r j}\right)$-expansions for conditional probabilities $\tilde{p}_{r j}(\varepsilon)$ by the $\left(l_{r j}^{-}, l_{r j}^{+}\right)$-expansions for transition probabilities $p_{r j}(\varepsilon)$ and the $\left(\hat{h}_{r j}, \hat{k}_{r j}\right)$ expansions for quantities $\hat{p}_{r j}(\varepsilon)$ by the $\left(l_{i r}^{-}, l_{i r}^{+}\right)$-expansions for transition probabilities $p_{i r}(\varepsilon)$. These are the only changes in the algorithm for construction of asymptotic expansions for expectations ${ }_{r} e_{i j}(\varepsilon), i, j \in{ }_{r} \mathbb{X}$ given in Steps 5.1 -5.8 , which are required.

The above remarks can be summarized in the following theorem.

Theorem 4. Conditions A - F assumed to hold for the semi-Markov process $\eta^{(\varepsilon)}(t)$, also hold for the reduced semi-Markov process ${ }_{r} \eta^{(\varepsilon)}(t)$, for every $r \in \mathbb{X}$. The asymptotic expansions penetrating conditions $\mathbf{B}$ and $\mathbf{F}$ are given for transition probabilities ${ }_{r} p_{i j}(\varepsilon), j \in{ }_{r} \mathbb{Y}_{i}, i \in{ }_{r} \mathbb{X}, r \in \mathbb{X}$ and expectations ${ }_{r} e_{i j}(\varepsilon), j \in{ }_{r} \mathbb{Y}_{i}, i \in{ }_{r} \mathbb{X}, r \in \mathbb{X}$ in Algorithms 3 and 5 .

Algorithm 6. This is an algorithm for computing upper bounds for remainders in asymptotic expansions for expectations ${ }_{r} e_{i j}(\varepsilon), i, j \in{ }_{r} \mathbb{X}$.

6.1. To construct $\left(\hat{h}_{i r}, \hat{k}_{i r}, \hat{\delta}_{i r}, \hat{G}_{i r}, \hat{\varepsilon}_{i r}\right)$-expansions for quantities $\hat{p}_{i r}(\varepsilon)=$ $\frac{p_{i r}(\varepsilon)}{\bar{p}_{r r}(\varepsilon)}=\frac{p_{i r}(\varepsilon)}{1-p_{r r}(\varepsilon)}, i \in{ }_{r} \mathbb{X}$, using the $\left(l_{i r}^{-}, l_{i r}^{+}, \delta_{i j}, G_{i j}, \varepsilon_{i j}\right)$-expansions for transition probabilities $p_{i r}(\varepsilon)$ given in condition $\mathbf{B}^{\prime}$, the $\left(\bar{h}_{r}, \bar{k}_{r}, \bar{\delta}_{i r}, \bar{G}_{i r}, \bar{\varepsilon}_{i r}\right)$-expansion for the non-absorption probability $\bar{p}_{r}(\varepsilon)=1-p_{r r}(\varepsilon)$ given in Algorithm 2, and proposition ( $\mathbf{v}$ ) (the division rule) of Lemma 4. In this case, parameters $\hat{\delta}_{i r}, \hat{G}_{i r}, \hat{\varepsilon}_{i r}, i \in{ }_{r} \mathbb{X}$ are given by the corresponding variants of relation (17).

6.2. To construct $\left(\check{h}_{i r j}, \check{k}_{i r j}, \check{\delta}_{i r j}, \check{G}_{i r j}, \check{\varepsilon}_{i r j}\right)$-expansions for products $\check{p}_{i r j}(\varepsilon)$ $=\hat{p}_{i r}(\varepsilon) \tilde{p}_{r j}(\varepsilon)=\frac{p_{i r}(\varepsilon)}{1-p_{r r}(\varepsilon)} \frac{p_{r j}(\varepsilon)}{1-p_{r r}(\varepsilon)}, j \in \mathbb{Y}_{i r}^{-}, i \in{ }_{r} \mathbb{X}, \operatorname{using}\left(\hat{h}_{i r}, \hat{k}_{i r}, \hat{\delta}_{i r}, \hat{G}_{i r}, \hat{\varepsilon}_{i r}\right)-$ expansions given in the above Step 6.1 , the $\left(\tilde{h}_{r j}, \tilde{k}_{r j}, \tilde{\delta}_{r j}, \tilde{G}_{r j}, \tilde{\varepsilon}_{r j}\right)$-expansions 
for conditional probabilities $\tilde{p}_{r j}(\varepsilon)=\frac{p_{r j}(\varepsilon)}{1-p_{r r}(\varepsilon)}$ given in Step 4.1 of Algorithm 4, and the proposition (iii) (the multiplication rule) of Lemma 4. In this case, parameters $\check{\delta}_{i r j}, \check{G}_{i r j}, \check{\varepsilon}_{i r j}, j \in \mathbb{Y}_{i r}^{-}, i \in{ }_{r} \mathbb{X}$ are given by the corresponding variants of relation (15).

6.3. To construct $\left(\dot{\tilde{h}}_{i r j}, \dot{\tilde{\kappa}}_{i r j}, \dot{\tilde{\delta}}_{i r j}, \dot{\tilde{G}}_{i r j}, \dot{\tilde{\varepsilon}}_{i r j}\right)$-expansions for products $\tilde{e}_{i r j}(\varepsilon)$ $=e_{i r}(\varepsilon) \tilde{p}_{r j}(\varepsilon)=e_{i r}(\varepsilon) \frac{p_{r j}(\varepsilon)}{1-p_{r r}(\varepsilon)}, j \in \mathbb{Y}_{i r}^{-}, i \in{ }_{r} \mathbb{X}$, using the $\left(m_{i r}^{-}, m_{i r}^{+}, \delta_{i r}, G_{i r}\right.$, $\left.\varepsilon_{i r}\right)$-expansions for expectations $e_{i r}(\varepsilon)$ given in condition $\mathbf{F}^{\prime}$, the $\left(\tilde{h}_{r j}, \tilde{k}_{r j}, \tilde{\delta}_{r j}\right.$, $\left.\tilde{G}_{r j}, \tilde{\varepsilon}_{r j}\right)$-expansions for conditional probabilities $\tilde{p}_{r j}(\varepsilon)=\frac{p_{r j}(\varepsilon)}{1-p_{r r}(\varepsilon)}$ given in Step 4.1 of Algorithm 4, and the proposition (iii) (the multiplication rule) of Lemma 4. In this case, parameters $\dot{\tilde{\delta}}_{i r j}, \dot{\tilde{G}}_{i r j}, \dot{\tilde{\varepsilon}}_{i r j}, j \in \mathbb{Y}_{i r}^{-}, i \in{ }_{r} \mathbb{X}$ are given by the corresponding variants of relation (15).

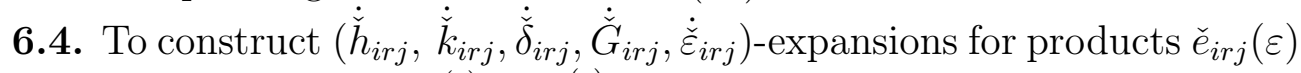
$=e_{r r}(\varepsilon) \cdot \check{p}_{i r j}(\varepsilon)=e_{r r}(\varepsilon) \frac{p_{i r}(\varepsilon)}{1-p_{r r}(\varepsilon)} \frac{p_{r j}(\varepsilon)}{1-p_{r r}(\varepsilon)}, j \in \mathbb{Y}_{i r}^{-}, i \in{ }_{r} \mathbb{X}$, using the $\left(m_{r r}^{-}, m_{r r}^{+}\right.$, $\left.\delta_{r r}, G_{r r}, \varepsilon_{r r}\right)$-expansions for expectations $e_{r r}(\varepsilon)$ given in condition $\mathbf{F}^{\prime}$, the $\left(\check{h}_{i r j}, \check{k}_{i r j}, \check{\delta}_{i r j}, \check{G}_{i r j}, \check{\varepsilon}_{i r j}\right)$-expansions for quantities $\check{p}_{i r j}(\varepsilon)$ given in the above Step 6.2, and the proposition (iii) (the multiplication rule) of Lemma 4. In this case, parameters $\left.\dot{\tilde{\delta}}_{i r j}, \dot{\mathscr{G}}_{i r j}, \dot{\check{\varepsilon}}_{i r j}\right), j \in \mathbb{Y}_{i r}^{-}, i \in{ }_{r} \mathbb{X}$ are given by the corresponding variants of relation (15).

6.5. To construct $\left(\dot{\hat{\hat{h}}}_{i r j}, \dot{\hat{\hat{k}}}_{i r j}, \dot{\hat{\hat{\delta}}}_{i r j}, \dot{\hat{G}}_{i r j}, \dot{\hat{\varepsilon}}_{i r j}\right)$-expansions for products $\hat{e}_{i r j}(\varepsilon)$ $=e_{r j}(\varepsilon) \cdot \hat{p}_{i r}(\varepsilon)=e_{r j}(\varepsilon) \frac{p_{i r}(\varepsilon)}{1-p_{r r}(\varepsilon)}, j \in \mathbb{Y}_{i r}^{-}, i \in{ }_{r} \mathbb{X}$, using the $\left(m_{r j}^{-}, m_{r j}^{+}, \delta_{r j}, G_{r j}\right.$, $\left.\varepsilon_{r j}\right)$-expansions for expectations $e_{r j}(\varepsilon)$ given in condition $\mathbf{F}^{\prime}$, the $\left(\hat{h}_{i r}, \hat{k}_{i r}, \hat{\delta}_{i r}\right.$, $\hat{G}_{i r}, \hat{\varepsilon}_{i r}$ )-expansions for quantities $\hat{p}_{i r}(\varepsilon)$ given in the above Step 6.1, and the proposition (iii) (the multiplication rule) of Lemma 4. In this case, parameters $\left.\dot{\hat{\delta}}_{i r j}, \dot{\hat{G}}_{i r j}, \dot{\hat{\varepsilon}}_{i r j}\right), j \in \mathbb{Y}_{i r}^{-}, i \in{ }_{r} \mathbb{X}$ are given by the corresponding variants of relation (15).

6.6. To construct $\left(\ddot{h}_{i r j}, \ddot{k}_{i r j}, \ddot{\delta}_{i r j}, \ddot{G}_{i r j}, \ddot{\varepsilon}_{i r j}\right)$-expansions for sums $\ddot{e}_{i r j}(\varepsilon)=$ $\tilde{e}_{i r j}(\varepsilon)+\check{e}_{i r j}(\varepsilon)+\hat{e}_{i r j}(\varepsilon)=e_{i r}(\varepsilon) \frac{p_{r j}(\varepsilon)}{1-p_{r r}(\varepsilon)}+e_{r r}(\varepsilon) \frac{p_{i r}(\varepsilon)}{1-p_{r r}(\varepsilon)} \frac{p_{r j}(\varepsilon)}{1-p_{r r}(\varepsilon)}+e_{r j}(\varepsilon) \frac{p_{i r}(\varepsilon)}{1-p_{r r}(\varepsilon)}$, using the $\left(\dot{\tilde{h}}_{i r j}, \dot{\tilde{k}}_{i r j}, \dot{\tilde{\delta}}_{i r j}, \dot{\tilde{G}}_{i r j}, \dot{\tilde{\varepsilon}}_{i r j}\right)$-expansions for quantities $\tilde{e}_{i r j}(\varepsilon)$, the $\left(\dot{\tilde{h}}_{i r j}\right.$, $\left.\dot{\tilde{k}}_{i r j}, \dot{\check{\delta}}_{i r j}, \dot{\mathscr{G}}_{i r j}, \dot{\varepsilon}_{i r j}\right)$-expansions for quantities $\check{e}_{i r j}(\varepsilon)$ and the $\left(\dot{\hat{h}}_{i r j}, \dot{\hat{k}}_{i r j}, \dot{\hat{\hat{\delta}}}_{i r j}\right.$, $\left.\dot{\hat{G}}_{i r j}, \dot{\hat{\varepsilon}}_{i r j}\right)$-expansions for quantities $\hat{e}_{i r j}(\varepsilon)$ given, respectively, in the above Steps 6.3, 6.4 and 6.5, and the proposition (i) (the summation rule) of Lemma 6. In this case, parameters $\ddot{\delta}_{i r j}, \ddot{G}_{i r j}, \ddot{\varepsilon}_{i r j}, j \in \mathbb{Y}_{i r}^{-}, i \in{ }_{r} \mathbb{X}$ are given by the corresponding variants of relation (24). 
6.7. To construct $\left(\dddot{h}_{i r j}, \dddot{k}_{i r j}, \dddot{\delta}_{i r j}, \dddot{G}_{i r j}, \dddot{\varepsilon}_{i r j}\right)$-expansions for sums $\dddot{e}_{i r j}(\varepsilon)$ $=e_{i j}(\varepsilon)+\ddot{e}_{i r j}(\varepsilon)=e_{i j}(\varepsilon)+e_{i r}(\varepsilon) \frac{p_{r j}(\varepsilon)}{1-p_{r r}(\varepsilon)}+e_{r r}(\varepsilon) \frac{p_{i r}(\varepsilon)}{1-p_{r r}(\varepsilon)} \frac{p_{r j}(\varepsilon)}{1-p_{r r}(\varepsilon)}+e_{r j}(\varepsilon) \frac{p_{i r}(\varepsilon)}{1-p_{r r}(\varepsilon)}$, using $\left(m_{i j}^{-}, m_{i j}^{+}, \dot{\delta}_{i j}, \dot{G}_{i j}, \dot{\varepsilon}_{i j}\right)$-expansions for expectations $e_{i j}(\varepsilon)$ given in condition $\mathbf{F}^{\prime}$, the $\left(\ddot{h}_{i r j}, \ddot{k}_{i r j}, \ddot{\delta}_{i r j}, \ddot{G}_{i r j}, \ddot{\varepsilon}_{i r j}\right)$-expansions for quantities $\ddot{e}_{i r j}(\varepsilon)$ given in the above Step 6.6, and the proposition (ii) (the summation rule) of Lemma 4. In this case, parameters $\dddot{\delta}_{i r j}, \dddot{G}_{i r j}, \dddot{\varepsilon}_{i r j}, j \in \mathbb{Y}_{i r}^{-}, i \in{ }_{r} \mathbb{X}$ are given by the corresponding variants of relation (14).

6.8. To construct $\left({ }_{r} m_{i j}^{-},{ }_{r} m_{i j}^{+},{ }_{r} \dot{\delta}_{i j},{ }_{r} \dot{G}_{i j},{ }_{r} \dot{\varepsilon}_{i j}\right)$-expansions expansions for expectations ${ }_{r} e_{i j}(\varepsilon)=\sum_{l={ }_{r} m_{i j}^{-}}^{r m_{i j}^{+}} b_{i j}[l] \varepsilon^{l}+o\left(\varepsilon^{r} m_{i j}^{+}\right), i, j \in{ }_{r} \mathbb{X}$, using the $\left(m_{i j}^{-}\right.$, $\left.m_{i j}^{+}, \dot{\delta}_{i j}, \dot{G}_{i j}, \dot{\varepsilon}_{i j}\right)$-expansions for expectations $e_{i j}(\varepsilon)$ given in condition $\mathbf{F}^{\prime}$, the $\left(\ddot{h}_{i r j}, \ddot{k}_{i r j}, \ddot{\delta}_{i r j}, \ddot{G}_{i r j}, \ddot{\varepsilon}_{i r j}\right)$-expansions for quantities $\ddot{e}_{i r j}(\varepsilon)$ and the $\left(\dddot{h}_{i r j}, \dddot{k}_{i r j}\right.$, $\left.\dddot{\delta}_{i r j}, \dddot{G}_{i r j}, \dddot{\varepsilon}_{i r j}\right)$-expansions for quantities $\dddot{e}_{i r j}(\varepsilon)$ given, respectively, in Steps 6.6 and 6.7, and the corresponding variants of formulas for expectations ${ }_{r} e_{i j}(\varepsilon)$ given in relation (103). In this case, parameters ${ }_{r} \dot{\delta}_{i j}=\dddot{\delta}_{i r j},{ }_{r} \dot{G}_{i j}=$ $\dddot{G}_{i r j},{ }_{r} \dot{\varepsilon}_{i j}=\dddot{\varepsilon}_{i r j}$ if $j \in \mathbb{Y}_{i r}^{+} \cap \mathbb{Y}_{i r}^{-}, i \in{ }_{r} \mathbb{X}$, or ${ }_{r} \dot{\delta}_{i j}=\delta_{i j},{ }_{r} \dot{G}_{i j}=G_{i j},{ }_{r} \dot{\varepsilon}_{i j}=\varepsilon_{i j}$ if $j \in \mathbb{Y}_{i r}^{+} \cap \overline{\mathbb{Y}}_{i r}^{-}, i \in{ }_{r} \mathbb{X}$, or ${ }_{r} \dot{\delta}_{i j}=\ddot{\delta}_{i r j},{ }_{r} \dot{G}_{i j}=\ddot{G}_{i r j},{ }_{r} \dot{\varepsilon}_{i j}=\ddot{\varepsilon}_{i r j}$ if $j \in$ $\overline{\mathbb{Y}}_{i r}^{+} \cap \mathbb{Y}_{i r}^{-}, i \in r_{r} \mathbb{X}$.

Case 2: $r \in \overline{\mathbb{Y}}$.

6.9. The corresponding algorithm is a particular case of the algorithm given in Steps 5.1 - 5.8. In this case the non-absorption probability $\bar{p}_{r r}(\varepsilon)=$ $1-p_{r r}(\varepsilon) \equiv 1$ and, thus, conditional probabilities $\tilde{p}_{r j}(\varepsilon)=\frac{p_{r j}(\varepsilon)}{1-p_{r r}(\varepsilon)}=p_{r j}(\varepsilon)$, $j \in \mathbb{Y}_{r r}^{+}$and quantities $\hat{p}_{i r}(\varepsilon)=\frac{p_{i r}(\varepsilon)}{1-p_{r r}(\varepsilon)}=p_{i r}(\varepsilon), i \in{ }_{r} \mathbb{X}$. This permits one replace the $\left(\tilde{h}_{r j}, \tilde{k}_{r j}, \tilde{\delta}_{r j}, \tilde{G}_{r j}, \tilde{\varepsilon}_{r j}\right)$-expansions for conditional probabilities $\tilde{p}_{r j}(\varepsilon)$ by the $\left(l_{r j}^{-}, l_{r j}^{+}, \delta_{r j}, G_{r j}, \varepsilon_{r j}\right)$-expansions for transition probabilities $p_{r j}(\varepsilon)$ and the $\left(\hat{h}_{r j}, \hat{k}_{r j}, \hat{\delta}_{r j}, \hat{G}_{r j}, \hat{\varepsilon}_{r j}\right)$-expansions for quantities $\hat{p}_{r j}(\varepsilon)$ by the $\left(l_{i r}^{-}, l_{i r}^{+}, \delta_{i r}, G_{i r}, \varepsilon_{i r}\right)$-expansions for transition probabilities $p_{i r}(\varepsilon)$. These are the only changes in the algorithm for construction of asymptotic expansions for expectations ${ }_{r} e_{i j}(\varepsilon), i, j \in{ }_{r} \mathbb{X}$ given in Steps $6.1-6.8$, which are required.

The above remarks can be summarized in the following theorem.

Theorem 5. Conditions A, $\mathbf{B}^{\prime}, \mathbf{C}-\mathbf{E}, \mathbf{F}^{\prime}$ assumed to hold for the semiMarkov process $\eta^{(\varepsilon)}(t)$, also hold for the reduced semi-Markov process ${ }_{r} \eta^{(\varepsilon)}(t)$, for every $r \in \mathbb{X}$. The upper bounds for remainders in expansions penetrating conditions $\mathbf{B}^{\prime}$ and $\mathbf{F}^{\prime}$ are given for transition probabilities ${ }_{r} p_{i j}(\varepsilon), j \in{ }_{r} \mathbb{Y}_{i}, i \in$ ${ }_{r} \mathbb{X}, r \in \mathbb{X}$ and expectations ${ }_{r} e_{i j}(\varepsilon), j \in{ }_{r} \mathbb{Y}_{i}, i \in{ }_{r} \mathbb{X}, r \in \mathbb{X}$ in Algorithms 4 
and 6 .

7. Sequential reduction of phase space and asymptotic expansions for stationary distributions of perturbed semi-Markov processes

In this section, we present algorithms of sequential reduction of phase spaces for semi-Markov processes and construction of asymptotic expansions for their stationary distributions.

7.1. Algorithms of sequential reduction of phase spaces for semiMarkov processes. Let $\eta^{(\varepsilon)}(t)$ be a semi-Markov process with the phase space $\mathbb{X}=\{1, \ldots, N\}$, which satisfy conditions $\mathbf{A}-\mathbf{F}$.

Let $\left\langle r_{1}, \ldots, r_{N}\right\rangle$ is a permutation of the sequence $\langle 1, \ldots, N\rangle$, and $\bar{r}_{n}=$ $\left(r_{1}, \ldots, r_{n}\right), n=1, \ldots, N$ is the corresponding sequence of growing chains of states from space $\mathbb{X}$.

Let us choose state $i \in \mathbb{X}$, and a permutation $\left\langle r_{1}, \ldots, r_{N}\right\rangle$ such that $r_{N}=i$.

Let us also assume that initial distribution $\bar{p}(\varepsilon)$ is concentrated in the state $i$, i.e., $p_{i}^{(\varepsilon)}=1$.

Algorithm 7. This is an algorithm for sequential reduction of the phase space for the semi-Markov process $\eta^{(\varepsilon)}(t)$ and constructing asymptotic expansions for transition probabilities and expectation of sojourn times for semi-Markov processes with reduced phase spaces.

7.1. Let $\bar{r}_{1} \eta^{(\varepsilon)}(t)={ }_{r_{1}} \eta^{(\varepsilon)}(t)$ be the reduced semi-Markov process which is the result of reduction of state $r_{1}$ for the semi-Markov process $\eta^{(\varepsilon)}(t)$. This semi-Markov process has the phase space $\bar{r}_{1} \mathbb{X}=\mathbb{X} \backslash\left\{r_{1}\right\}$, transition probabilities of the embedded Markov chain ${ }_{\bar{r}_{1}} p_{i^{\prime} j^{\prime}}(\varepsilon), i^{\prime}, j^{\prime} \in{ }_{r_{1}} \mathbb{X}$ and expectations of transition times $\bar{r}_{\bar{r}_{1}} e_{i^{\prime} j^{\prime}}(\varepsilon), i^{\prime}, j^{\prime} \in{ }_{r_{1}} \mathbb{X}$, which are determined by the transition probabilities and the expectations of transition times for the process $\eta^{(\varepsilon)}(t)$ via relations (87) and (96). According Theorem 1, the expectations of hitting times $E_{i^{\prime} j^{\prime}}(\varepsilon), i^{\prime}, j^{\prime} \in \bar{r}_{1} \mathbb{X}$ coincide for the semi-Markov processes $\eta^{(\varepsilon)}(t)$ and $\bar{r}_{1} \eta^{(\varepsilon)}(t)$. According Theorems 2 and 4, the semi-Markov process $\bar{r}_{1} \eta^{(\varepsilon)}(t)$ satisfy conditions $\mathbf{A}-\mathbf{F}$. The transition sets $\bar{r}_{1} \mathbb{Y}_{i^{\prime}}={ }_{r_{1}} \mathbb{Y}_{i^{\prime}}, i^{\prime} \in \bar{r}_{1} \mathbb{X}$ are determined for the process $\bar{r}_{1} \eta^{(\varepsilon)}(t)$ by condition $\mathbf{A}$ and relation (90). Therefore, the $\left(\bar{r}_{1} l_{i^{\prime} j^{\prime}}^{-}, \bar{r}_{1} l_{i^{\prime} j^{\prime}}^{+}\right)$-expansions for transition probabilities $\bar{r}_{1} p_{i^{\prime} j^{\prime}}(\varepsilon), j^{\prime} \in$ $\bar{r}_{1} \mathbb{Y}_{i^{\prime}}, i^{\prime} \in \bar{r}_{1} \mathbb{X}$ and $\left(\bar{r}_{1} m_{i^{\prime} j^{\prime}}^{-}, \bar{r}_{1} m_{i^{\prime} j^{\prime}}^{+}\right)$-expansions for expectations $\bar{r}_{1} e_{i^{\prime} j^{\prime}}(\varepsilon), j^{\prime} \in$ $\bar{r}_{1} \mathbb{Y}_{i^{\prime}}, i^{\prime} \in \bar{r}_{1} \mathbb{X}$ can be constructed by applying Algorithms 1,3 and 5 to 
the $\left(l_{i^{\prime} j^{\prime}}^{-}, l_{i^{\prime} j^{\prime}}^{+}\right)$-expansions for transition probabilities $p_{i^{\prime} j^{\prime}}(\varepsilon), j^{\prime} \in \mathbb{Y}_{i^{\prime}}, i^{\prime} \in \mathbb{X}$ and $\left(m_{i^{\prime} j^{\prime}}^{-}, m_{i^{\prime} j^{\prime}}^{+}\right)$-expansions for expectations $e_{i^{\prime} j^{\prime}}(\varepsilon), j^{\prime} \in \mathbb{Y}_{i^{\prime}}, i^{\prime} \in \mathbb{X}$. These expansions ara pivotal.

7.2. Let $\bar{r}_{2} \eta^{(\varepsilon)}(t)$ be the reduced semi-Markov process which is the result of reduction of state $r_{2}$ for the semi-Markov process $\bar{r}_{1} \eta^{(\varepsilon)}(t)$. This semiMarkov process has the phase space $\bar{r}_{2} \mathbb{X}=\mathbb{X} \backslash\left\{r_{1}, r_{2}\right\}$, the transition probabilities of the embedded Markov chain $\bar{r}_{2} p_{i^{\prime} j^{\prime}}(\varepsilon), i^{\prime}, j^{\prime} \in_{r_{1}} \mathbb{X}$ and the expectations of transition times $\bar{r}_{2} e_{i^{\prime} j^{\prime}}(\varepsilon), i^{\prime}, j^{\prime} \in r_{2} \mathbb{X}$, which are determined by the transition probabilities and the expectations of transition times for the process $\bar{r}_{1} \eta^{(\varepsilon)}(t)$ via relations (87) and (196). According Theorem 1 , the expectations of hitting times $E_{i^{\prime} j^{\prime}}(\varepsilon), i^{\prime}, j^{\prime} \in \bar{r}_{2} \mathbb{X}$ coincide for the semi-Markov processes $\eta^{(\varepsilon)}(t), \bar{r}_{1} \eta^{(\varepsilon)}(t)$ and $\bar{r}_{2} \eta^{(\varepsilon)}(t)$. According Theorems 2 and 4 , the transition probabilities of the embedded Markov chain $\bar{r}_{2} p_{i^{\prime} j^{\prime}}(\varepsilon), i^{\prime}, j^{\prime} \in \bar{r}_{2} \mathbb{X}$ and the expectations of transition times $\bar{r}_{2} e_{i^{\prime} j^{\prime}}(\varepsilon), i^{\prime}, j^{\prime} \in \bar{r}_{2} \mathbb{X}$ satisfy conditions $\mathbf{A}-\mathbf{F}$. The transition sets $\bar{r}_{2} \mathbb{Y}_{i^{\prime}}, i^{\prime} \in \bar{r}_{2} \mathbb{X}$ are determined for the process $\bar{r}_{2} \eta^{(\varepsilon)}(t)$ by condition $\mathbf{A}$ and relation (90) in the same way as the transition sets $\bar{r}_{1} \mathbb{Y}_{i^{\prime}}, i^{\prime} \in{ }_{r_{1}} \mathbb{X}$ are determined by condition $\mathbf{A}$ and relation (90) for the process $\bar{r}_{1} \eta^{(\varepsilon)}(t)$. Therefore, the $\left(\bar{r}_{2} l_{i^{\prime} j^{\prime}}^{-}, \bar{r}_{2} l_{i^{\prime} j^{\prime}}^{+}\right)$-expansions for transition probabilities $\bar{r}_{2} p_{i^{\prime} j^{\prime}}(\varepsilon), j^{\prime} \in \bar{r}_{2} \mathbb{Y}_{i^{\prime}}, i^{\prime} \in \bar{r}_{2} \mathbb{X}$ and $\left(\bar{r}_{2} m_{i^{\prime} j^{\prime}}^{-}, \bar{r}_{2} m_{i^{\prime} j^{\prime}}^{+}\right)$-expansions for expectations $\bar{r}_{2} e_{i^{\prime} j^{\prime}}(\varepsilon), j^{\prime} \in \bar{r}_{2} \mathbb{Y}_{i^{\prime}}, i^{\prime} \in \bar{r}_{2} \mathbb{X}$ can be constructed by applying Algorithms 1, 3 and 5 to the $\left(\bar{r}_{1} l_{i^{\prime} j^{\prime}}^{-}, \bar{r}_{1} l_{i^{\prime} j^{\prime}}^{+}\right)$-expansions for transition probabilities $\bar{r}_{1} p_{i^{\prime} j^{\prime}}(\varepsilon), j^{\prime} \in \bar{r}_{1} \mathbb{Y}_{i^{\prime}}, i^{\prime} \in \bar{r}_{1} \mathbb{X}$ and $\left(\bar{r}_{1} m_{i^{\prime} j^{\prime}}^{-}, \bar{r}_{1} m_{i^{\prime} j^{\prime}}^{+}\right)$-expansions for expectations $\bar{r}_{1} e_{i^{\prime} j^{\prime}}(\varepsilon), j^{\prime} \in{ }_{\bar{r}_{1}} \mathbb{Y}_{i^{\prime}}, i^{\prime} \in{ }_{\bar{r}_{1}} \mathbb{X}$. These expansions ara pivotal.

7.3. By continuing the above procedure of phase space reduction for states $r_{3}, \ldots, r_{N-1}$, we construct the semi-Markov process $\bar{r}_{N-1} \eta^{(\varepsilon)}(t)$ with the phase space $\bar{r}_{N-1} \mathbb{X}=\mathbb{X} \backslash\left\{r_{1}, r_{2}, \ldots, r_{N-1}\right\}=\{i\}$ (which is a one-point set), the transition probabilities of the embedded Markov chain ${ }_{\bar{r}_{N-1}} p_{i i}(\varepsilon)=1$, and the expectations of transition times $\bar{r}_{N-1} e_{i i}(\varepsilon)$, which are determined by the transition probabilities and the expectations of transition times of the process $\bar{r}_{N-2} \eta^{(\varepsilon)}(t)$ via relations (87) and (96). According Theorem 1, the expectations of hitting times $E_{i i}(\varepsilon)$ for the semi-Markov processes $\eta^{(\varepsilon)}(t)$, $\bar{r}_{1} \eta^{(\varepsilon)}(t), \ldots, \bar{r}_{N-1} \eta^{(\varepsilon)}(t)$ coincide. According Theorems 2 and 4 , the transition probabilities of the embedded Markov chain $\bar{r}_{N-1} p_{i i}(\varepsilon)=1$ and the expectations of transition times $\bar{r}_{N-1} e_{i i}(\varepsilon)$ satisfy conditions $\mathbf{A}-\mathbf{F}$. In this case, the transition set $\bar{r}_{N-1} \mathbb{Y}_{i}=\{i\}$, for every $i \in \mathbb{X}$. Therefore, the $\left(\bar{r}_{N-1} l_{i^{\prime} j^{\prime}}^{-}, \bar{r}_{N-1} l_{i^{\prime} j^{\prime}}^{+}\right)$-expansions for transition probabilities $\bar{r}_{N-1} p_{i^{\prime} j^{\prime}}(\varepsilon)=1, j^{\prime} \in$ $\bar{r}_{N-1} \mathbb{Y}_{i^{\prime}}, i^{\prime} \in \bar{r}_{N-1} \mathbb{X}$ (which take the form of relation (58)) and ${\left(\bar{r}_{N-1}\right.}_{m_{i^{\prime} j^{\prime}}}$, 
$\left.\bar{r}_{N-1} m_{i^{\prime} j^{\prime}}^{+}\right)$-expansions for expectations $\bar{r}_{N-1} e_{i^{\prime} j^{\prime}}(\varepsilon), j^{\prime} \in \bar{r}_{N-1} \mathbb{Y}_{i^{\prime}}, i^{\prime} \in \bar{r}_{N-1} \mathbb{X}$ can be constructed by applying Algorithms 1, 3 and 5 to the $\left(\bar{r}_{N-2} l_{i^{\prime} j^{\prime}}^{-}, \bar{r}_{N-2} l_{i^{\prime} j^{\prime}}^{+}\right)$ -expansions for transition probabilities $\bar{r}_{N-2} p_{i^{\prime} j^{\prime}}(\varepsilon), j^{\prime} \in \bar{r}_{N-2} \mathbb{Y}_{i^{\prime}}, i^{\prime} \in{ }_{\bar{r}_{N-2}} \mathbb{X}$ and $\left(_{\bar{r}_{N-2}} m_{i^{\prime} j^{\prime}}^{-}, \bar{r}_{N-2} m_{i^{\prime} j^{\prime}}^{+}\right)$-expansions for expectations ${ }_{\bar{r}_{N-2}} e_{i^{\prime} j^{\prime}}(\varepsilon), j^{\prime} \in \bar{r}_{N-2} \mathbb{Y}_{i^{\prime}}$, $i^{\prime} \in \bar{r}_{N-2} \mathbb{X}$. These expansions ara pivotal.

7.4. The semi-Markov process $\bar{r}_{N-1} \eta^{(\varepsilon)}(t)$ has the one-point phase space $\bar{r}_{N-1} \mathbb{X}=\{i\}$ and, thus, the transition probability $\bar{r}_{N-1} p_{i i}(\varepsilon) \equiv 1$, while the expectation of transition time $\bar{r}_{N-1} e_{i i}(\varepsilon)=E_{i i}(\varepsilon)$. The above algorithm of sequential reduction of phase space should be repeated for every $i \in \mathbb{X}$. In this way, the Laurent asymptotic expansions for quantities $E_{i i}(\varepsilon), i \in \mathbb{X}$ can be written down. These asymptotic expansions have the following form,

$$
E_{i i}(\varepsilon)=\sum_{l=M_{i i}^{-}}^{M_{i i}^{+}} B_{i i}[l] \varepsilon^{l}+\hat{o}_{i}\left(\varepsilon^{M_{i i}^{+}}\right), i \in \mathbb{X}
$$

where parameters $M_{i i}^{ \pm}={ }_{\bar{r}_{N-1}} m_{i i}^{ \pm}, i \in \mathbb{X}$ and the coefficients $B_{i i}[l]={ }_{\bar{r}_{N-1}} b_{i i}[l]$, $l=M_{i i}^{-}, \ldots, M_{i i}^{+}, i \in \mathbb{X}$, where $\bar{r}_{N-1} b_{i i}[l]$ are coefficients in the correspond$\operatorname{ing}\left({ }_{\bar{r}_{N-1}} m_{i^{\prime} j^{\prime}}^{-}, \bar{r}_{N-1} m_{i^{\prime} j^{\prime}}^{+}\right)$-expansions for expectations ${ }_{\bar{r}_{N-1}} e_{i^{\prime} j^{\prime}}(\varepsilon), j^{\prime} \in{ }_{\bar{r}_{N-1}} \mathbb{Y}_{i^{\prime}}$, $i^{\prime} \in \bar{r}_{N-1} \mathbb{X}$. These expansions are pivotal.

It should be noted that, for every $n=1, \ldots, N-1$, the reduced semiMarkov process $\bar{r}_{n} \eta^{(\varepsilon)}(t)$ is invariant with respect to any permutation $\bar{r}_{n}^{\prime}=$ $\left(r_{1}^{\prime}, \ldots, r_{n}^{\prime}\right)$ of the the sequence $\bar{r}_{n}=\left(r_{1}, \ldots, r_{n}\right)$.

Indeed, for every such permutation $\bar{r}_{n}^{\prime}=\left(r_{1}^{\prime}, \ldots, r_{n}^{\prime}\right)$, the corresponding reduced semi-Markov process $\bar{r}_{n}^{\prime} \eta^{(\varepsilon)}(t)$ is constructed from the initial semiMarkov process $\eta^{(\varepsilon)}(t)$, as the sequence of its states at sequential moment of hitting into the same reduced phase space $\bar{r}_{n}^{\prime} \mathbb{X}=\mathbb{X} \backslash\left\{r_{1}^{\prime}, \ldots, r_{n}^{\prime}\right\}={ }_{r_{n}} \mathbb{X}=$ $\mathbb{X} \backslash\left\{r_{1}, \ldots, r_{n}\right\}$ and times between sequential jumps of the reduced semiMarkov process $\bar{r}_{n}^{\prime} \eta^{(\varepsilon)}(t)$ which are times between sequential hitting of the above reduced space by the initial semi-Markov process $\eta^{(\varepsilon)}(t)$.

This implies that the expectation of transition time $\bar{r}_{n} e_{i^{\prime} j^{\prime}}(\varepsilon)$ is, for every $i^{\prime}, j^{\prime} \in \bar{r}_{n} \mathbb{X}$ and $n=1, \ldots, N-1$, invariant with respect to any permutation $\bar{r}_{n}^{\prime}=\left(r_{1}^{\prime}, \ldots, r_{n}^{\prime}\right)$ of the sequence $\bar{r}_{n}=\left(r_{1}, \ldots, r_{n}\right)$.

Moreover, as follows from the Algorithms 1 - 7, the expectation of transition time $\bar{r}_{n} e_{i^{\prime} j^{\prime}}(\varepsilon)$ is a rational function of initial transition probabilities $p_{i j}(\varepsilon), j \in \mathbb{Y}_{i}, i \in \mathbb{X}$ and expectations $e_{i j}(\varepsilon), j \in \mathbb{Y}_{i}, i \in \mathbb{X}$ (a quotient of two sums of products of some of these probabilities and expectations), which, according the above remarks, is invariant with respect to any permutation $\bar{r}_{n}^{\prime}=\left(r_{1}^{\prime}, \ldots, r_{n}^{\prime}\right)$ of the sequence $\bar{r}_{n}=\left(r_{1}, \ldots, r_{n}\right)$. 
By using identical arithmetical transformations (disclosure of brackets, imposition of a common factor out of the brackets, bringing a fractional expression to a common denominator, permutation of summands or multipliers, elimination of expression with equal absolute values and opposite signs in the sums and elimination of equal expressions in the quotients, etc.) the rational function ${\overline{r_{n}^{\prime}}}_{n} e_{i^{\prime} j^{\prime}}(\varepsilon)$ given by Algorithm 7 can be transformed in the rational function $\overline{\bar{r}}_{n} e_{i^{\prime} j^{\prime}}(\varepsilon)$ given by Algorithm 7 and wise versa.

By Lemma 8, these transformations do not affect the corresponding asymptotic expansions for expectation $\overline{\bar{r}}_{n} e_{i^{\prime} j^{\prime}}(\varepsilon)$ given by Algorithm 7, and, thus, these asymptotic expansions are invariant with respect to any permutation $\bar{r}_{n}^{\prime}=\left(r_{1}^{\prime}, \ldots, r_{n}^{\prime}\right)$ of the sequence $\bar{r}_{n}=\left(r_{1}, \ldots, r_{n}\right)$.

The above remarks can be summarized in the following theorem.

Theorem 6. Let conditions A - F hold for semi-Markov processes $\eta^{(\varepsilon)}(t)$. Then, for every $i \in \mathbb{X}$, the Laurent asymptotic expansion (104) for the expectation of hitting times $E_{i i}(\varepsilon)$ given by Algorithm 7 can be written down. This expansion is invariant with respect to the choice of permutation $\left\langle r_{1}, \ldots, r_{N-1}, i\right\rangle$ of sequence $\langle 1, \ldots, N\rangle$, in the above algorithm.

Let us now assume that conditions $\mathbf{A}, \mathbf{B}^{\prime}, \mathbf{C}-\mathbf{E}, \mathbf{F}^{\prime}$ hold for the semiMarkov process $\eta^{(\varepsilon)}(t)$.

Algorithm 8. This is an algorithm for computing upper bounds for remainders in asymptotic expansions for transition probabilities and expectation of sojourn times for semi-Markov processes with reduced phase spaces.

8.1. Let $\bar{r}_{1} \eta^{(\varepsilon)}(t)={ }_{r_{1}} \eta^{(\varepsilon)}(t)$ be be the reduced semi-Markov process, which is constructed as this is described in Step 7.1 of Algorithm 7. According Theorems 3 and 5, the semi-Markov process $\bar{r}_{1} \eta^{(\varepsilon)}(t)$ satisfies conditions $\mathbf{A}, \mathbf{B}^{\prime}, \mathbf{C}-\mathbf{E}, \mathbf{F}^{\prime}$. Therefore, $\left(\bar{r}_{1} l_{i^{\prime} j^{\prime}}^{-}, \bar{r}_{1} l_{i^{\prime} j^{\prime}}^{+}, \bar{r}_{1} \delta_{i^{\prime} j^{\prime}}, \bar{r}_{1} G_{i^{\prime} j^{\prime}}, \bar{r}_{1} \varepsilon_{i^{\prime} j^{\prime}}\right)$ expansions for transition probabilities $\bar{r}_{1} p_{i^{\prime} j^{\prime}}(\varepsilon), j^{\prime} \in \bar{r}_{1} \mathbb{Y}_{i^{\prime}}, i^{\prime} \in \bar{r}_{1} \mathbb{X}$ and $\left(\bar{r}_{1} m_{i^{\prime} j^{\prime}} \bar{r}_{1} m_{i^{\prime} j^{\prime}}, \bar{r}_{1} \dot{\delta}_{i^{\prime} j^{\prime}}, \bar{r}_{1} \dot{G}_{i^{\prime} j^{\prime}}, \bar{r}_{1} \dot{\varepsilon}_{i^{\prime} j^{\prime}}\right)$-expansions for expectations $\bar{r}_{1} e_{i^{\prime} j^{\prime}}(\varepsilon), j^{\prime}$ $\in \bar{r}_{1} \mathbb{Y}_{i^{\prime}}, i^{\prime} \in \bar{r}_{1} \mathbb{X}$ can be constructed by applying Algorithms $1-5$ to the $\left(l_{i^{\prime} j^{\prime}}^{-}, l_{i^{\prime} j^{\prime}}^{+}, \delta_{i^{\prime} j^{\prime}}, G_{i^{\prime} j^{\prime}}, \varepsilon_{i^{\prime} j^{\prime}}\right)$-expansions for transition probabilities $p_{i^{\prime} j^{\prime}}(\varepsilon), j^{\prime} \in$ $\mathbb{Y}_{i^{\prime}}, i^{\prime} \in \mathbb{X}$ and $\left(m_{i^{\prime} j^{\prime}}^{-}, m_{i^{\prime} j^{\prime}}^{+}, \dot{\delta}_{i^{\prime} j^{\prime}}, \dot{G}_{i^{\prime} j^{\prime}}, \dot{\varepsilon}_{i^{\prime} j^{\prime}}\right)$-expansions for expectations $e_{i^{\prime} j^{\prime}}(\varepsilon)$, $j^{\prime} \in \mathbb{Y}_{i^{\prime}}, i^{\prime} \in \mathbb{X}$.

8.2. Let $\bar{r}_{2} \eta^{(\varepsilon)}(t)$ be the reduced semi-Markov process, which is constructed as this is described in Step 7.2 of Algorithm 7. According Theorems 3 and 5, the semi-Markov process $\bar{r}_{2} \eta^{(\varepsilon)}(t)$ satisfies conditions $\mathbf{A}, \mathbf{B}^{\prime}, \mathbf{C}-$ E, $\mathbf{F}^{\prime}$. Therefore, $\left(\bar{r}_{2} l_{i^{\prime} j^{\prime}}^{-}, \bar{r}_{2} l_{i^{\prime} j^{\prime}}^{+}, \bar{r}_{2} \delta_{i^{\prime} j^{\prime}}, \bar{r}_{2} G_{i^{\prime} j^{\prime}}, \bar{r}_{2} \varepsilon_{i^{\prime} j^{\prime}}\right)$-expansions for transi- 
tion probabilities $\bar{r}_{2} p_{i^{\prime} j^{\prime}}(\varepsilon), j^{\prime} \in \bar{r}_{2} \mathbb{Y}_{i^{\prime}}, i^{\prime} \in \bar{r}_{2} \mathbb{X}$ and $\left(\bar{r}_{2} m_{i^{\prime} j^{\prime}}^{-}, \bar{r}_{2} m_{i^{\prime} j^{\prime}}^{+}, \bar{r}_{2} \dot{\delta}_{i^{\prime} j^{\prime}}\right.$, $\bar{r}_{2} \dot{G}_{i^{\prime} j^{\prime}}, \bar{r}_{2} \dot{\varepsilon}_{i^{\prime} j^{\prime}}$-expansions for expectations $\bar{r}_{2} e_{i^{\prime} j^{\prime}}(\varepsilon), j^{\prime} \in \bar{r}_{2} \mathbb{Y}_{i^{\prime}}, i^{\prime} \in \bar{r}_{2} \mathbb{X}$ can be constructed by applying Algorithms $1-5$ to the $\left(\bar{r}_{1} l_{i^{\prime} j^{\prime}}^{-}, \bar{r}_{1} l_{i^{\prime} j^{\prime}}^{+}, \bar{r}_{1} \delta_{i^{\prime} j^{\prime}}, \bar{r}_{1} G_{i^{\prime} j^{\prime}}\right.$, $\left.\bar{r}_{1} \varepsilon_{i^{\prime} j^{\prime}}\right)$-expansions for transition probabilities $p_{i^{\prime} j^{\prime}}(\varepsilon), j^{\prime} \in \mathbb{Y}_{i^{\prime}}, i^{\prime} \in \bar{r}_{1} \mathbb{X}$ and $\left(\bar{r}_{1} m_{i^{\prime} j^{\prime}}^{-}, \bar{r}_{1} m_{i^{\prime} j^{\prime}}^{+}, \bar{r}_{1} \dot{\delta}_{i^{\prime} j^{\prime}}, \bar{r}_{1} \dot{G}_{i^{\prime} j^{\prime}}, \bar{r}_{1} \dot{\varepsilon}_{i^{\prime} j^{\prime}}\right)$-expansions for expectations $e_{i^{\prime} j^{\prime}}(\varepsilon)$, $j^{\prime} \in \bar{r}_{1} \mathbb{Y}_{i^{\prime}}, i^{\prime} \in \bar{r}_{1} \mathbb{X}$.

8.3. Finally, let $\bar{r}_{N-1} \eta^{(\varepsilon)}(t)$ be the reduced semi-Markov process, which is constructed as this is described in Step 7.2 of Algorithm 7. According Theorems 3 and 5, the semi-Markov process $\bar{r}_{N-1} \eta^{(\varepsilon)}(t)$ satisfies conditions $\mathbf{A}, \mathbf{B}^{\prime}, \mathbf{C}-\mathbf{E}, \mathbf{F}^{\prime}$. Therefore, $\left({ }_{\bar{r}_{N-1}} l_{i^{\prime} j^{\prime}}^{-}, \bar{r}_{N-1} l_{i^{\prime} j^{\prime}}^{+}, \bar{r}_{N-1} \delta_{i^{\prime} j^{\prime}}, \bar{r}_{N-1} G_{i^{\prime} j^{\prime}}, \bar{r}_{N-1} \varepsilon_{i^{\prime} j^{\prime}}\right)-$ expansions for transition probabilities $\bar{r}_{N-1} p_{i^{\prime} j^{\prime}}(\varepsilon), j^{\prime} \in \bar{r}_{N-1} \mathbb{Y}_{i^{\prime}}, i^{\prime} \in \bar{r}_{N-1} \mathbb{X}$ and $\left(_{\bar{r}_{N-1}} m_{i^{\prime} j^{\prime}}^{-}, \bar{r}_{N-1} m_{i^{\prime} j^{\prime}}^{+}, \bar{r}_{N-1} \dot{\delta}_{i^{\prime} j^{\prime}}, \bar{r}_{N-1} \dot{G}_{i^{\prime} j^{\prime}}, \bar{r}_{N-1} \dot{\varepsilon}_{i^{\prime} j^{\prime}}\right)$-expansions for expectations $\bar{r}_{N-1} e_{i^{\prime} j^{\prime}}(\varepsilon), j^{\prime} \in \bar{r}_{N-1} \mathbb{Y}_{i^{\prime}}, i^{\prime} \in \bar{r}_{N-1} \mathbb{X}$ can be constructed by applying Algorithms $1-5$ to the $\left(\bar{r}_{N-2} l_{i^{\prime} j^{\prime}}^{-}, \bar{r}_{N-2} l_{i^{\prime} j^{\prime}}^{+}, \bar{r}_{N-2} \delta_{i^{\prime} j^{\prime}}, \bar{r}_{N-2} G_{i^{\prime} j^{\prime}}, \bar{r}_{N-2} \varepsilon_{i^{\prime} j^{\prime}}\right)-$ expansions for transition probabilities $p_{i^{\prime} j^{\prime}}(\varepsilon), j^{\prime} \in \mathbb{Y}_{i^{\prime}}, i^{\prime} \in \bar{r}_{N-2} \mathbb{X}$ and $\left(\bar{r}_{N-2} m_{i^{\prime} j^{\prime}}^{-}, \bar{r}_{N-2} m_{i^{\prime} j^{\prime}}^{+}, \bar{r}_{N-2} \dot{\delta}_{i^{\prime} j^{\prime}}, \bar{r}_{N-2} \dot{G}_{i^{\prime} j^{\prime}}, \bar{r}_{N-2} \dot{\varepsilon}_{i^{\prime} j^{\prime}}\right)$-expansions for expectations $e_{i^{\prime} j^{\prime}}(\varepsilon), j^{\prime} \in \bar{r}_{N-2} \mathbb{Y}_{i^{\prime}}, i^{\prime} \in \bar{r}_{N-2} \mathbb{X}$.

8.4. Finally, due to equalities $\bar{r}_{N-1} e_{i i}(\varepsilon)=E_{i i}(\varepsilon), i \in \mathbb{X}$, we get that the asymptotic expansion (104) for expectations $E_{i i}(\varepsilon), i \in \mathbb{X}$, given in the Step 7.4 of Algorithm 7 , is a $\left(M_{i i}^{-}, M_{i i}^{+}, \delta_{i i}^{\circ}, G_{i i}^{\circ}, \varepsilon_{i i}^{\circ}\right)$-expansion with parameters $M_{i i}^{-}={ }_{\bar{r}_{N-1}} m_{i i}^{-}, M_{i i}^{+}={ }_{\bar{r}_{N-1}} m_{i i}^{+}, \delta_{i i}^{\circ}=\bar{r}_{N-1} \dot{\delta}_{i i}, G_{i i}^{\circ}={ }_{\bar{r}_{N-1}} \dot{G}_{i i}, \varepsilon_{i i}^{\circ}={ }_{\bar{r}_{N-1}} \dot{\varepsilon}_{i i}$.

In this case, the invariance of explicit upper bounds for remainders given by Algorithm 8, with respect to the choice of any permutation $\left\langle r_{1}, \ldots, r_{N-1}, i\right\rangle$ of sequence $\langle 1, \ldots, N\rangle$, can not be guaranteed.

However, Lemma 9 guarantees that the following inequalities hold for the parameters $\delta_{i i}^{\circ}, i \in \mathbb{X}$,

$$
\delta_{i i}^{\circ} \geq \delta^{\circ}=\min _{j \in \mathbb{Y}_{i}, i \in \mathbb{X}}\left(\delta_{i j} \wedge \dot{\delta}_{i j}\right)
$$

The following theorem takes place.

Theorem 7. Let conditions $\mathbf{A}, \mathbf{B}^{\prime}, \mathbf{C}-\mathbf{E}, \mathbf{F}^{\prime}$ hold for semi-Markov processes $\eta^{(\varepsilon)}(t)$. Then, for every $i \in \mathbb{X}$, the $\left(M_{i i}^{-}, M_{i i}^{+}\right)$-expansion (104) for the expectations of hitting times $E_{i i}(\varepsilon)$, given by Algorithm 7 , is a $\left(M_{i i}^{-}, M_{i i}^{+}, \delta_{i i}^{\circ}\right.$, $\left.G_{i i}^{\circ}, \varepsilon_{i i}^{\circ}\right)$-expansion, with parameters $\delta_{i i}^{\circ}, G_{i i}^{\circ}, \varepsilon_{i i}^{\circ}$ given in Algorithm 8. The inequality (105) holds for parameters $\delta_{i i}^{\circ}, i \in \mathbb{X}$.

7.2. Asymptotic expansions for stationary probabilities of perturbed semi-Markov processes. Let us recall relation (78) for stationary 
probabilities of the semi-Markov process $\eta^{(\varepsilon)}(t)$,

$$
\pi_{i}(\varepsilon)=\frac{e_{i}(\varepsilon)}{E_{i i}(\varepsilon)}, i \in \mathbb{X} .
$$

Algorithm 9. This is an algorithm for constructing asymptotic expansions for stationary probabilities of perturbed semi-Markov processes.

9.1. Conditions $\mathbf{A}$ - F and proposition (i) (the multiple summation rule) of Lemma 5 , permits one can construct $\left(m_{i}^{-}, m_{i}^{+}\right)$-expansions for expectations $e_{i}(\varepsilon), i \in \mathbb{X}$, which take the following forms,

$$
\begin{aligned}
e_{i}(\varepsilon) & =\sum_{j \in \mathbb{Y}_{i}} e_{i j}(\varepsilon) \\
& =\sum_{j \in \mathbb{Y}_{i}}\left(\sum_{l=m_{i j}^{-}}^{m_{i}^{+}} b_{i j}[l] \varepsilon^{l}+\dot{o}_{i j}\left(\varepsilon^{m_{i j}^{+}}\right)\right) \\
& =\sum_{l=m_{i}^{-}}^{m_{i}^{+}} b_{i}[l] \varepsilon^{l}+\dot{o}_{i}\left(\varepsilon^{m_{i}^{+}}\right), \quad i \in \mathbb{X},
\end{aligned}
$$

where

$$
m_{i}^{-}=\min _{j \in \mathbb{Y}_{i}} m_{i j}^{-}, m_{i}^{+}=\min _{j \in \mathbb{Y}_{i}} m_{i j}^{+}, i \in \mathbb{X}
$$

and

$$
b_{i}\left[m_{i}^{-}+l\right]=\sum_{j \in Y_{i}} b_{i j}\left[m_{i}^{-}+l\right], l=0, \ldots, m_{i}^{+}-m_{i}^{-}, i \in \mathbb{X},
$$

where $b_{i j}\left[m_{i}^{-}+l\right]=0$, for $0 \leq l<m_{i j}^{-}-m_{i}^{-}, j \in Y_{i}, i \in \mathbb{X}$.

The above asymptotic expansions are pivotal for all $i \in \mathbb{X}$.

9.2. Conditions $\mathbf{A}-\mathbf{F}$, relation (106) and proposition (v) (the division rule) of Lemma 3 , permits us construct $\left(n_{i}^{-}, n_{i}^{+}\right)$-expansions for stationary probabilities $\pi_{i}(\varepsilon), i \in \mathbb{X}$, which take the following forms,

$$
\pi_{i}(\varepsilon)=\sum_{l=n_{i}^{-}}^{n_{i}^{+}} c_{i}[l] \varepsilon^{l}+o_{i}\left(\varepsilon^{n_{i}^{+}}\right), i \in \mathbb{X},
$$

where

$$
n_{i}^{-}=m_{i}^{-}-M_{i i}^{-}, n_{i}^{+}=\left(m_{i}^{+}-M_{i i}^{-}\right) \wedge\left(m_{i}^{-}+M_{i i}^{+}-2 M_{i i}^{-}\right), i \in \mathbb{X},
$$


and

$$
\begin{aligned}
c_{i}\left[n_{i}^{-}+l\right] & =\frac{b_{i}\left[m_{i}^{-}+l\right]-\sum_{1 \leq l^{\prime} \leq l} B_{i i}\left[M_{i i}^{-}+l\right] c_{i}\left[n_{i}^{-}+l-l^{\prime}\right]}{B_{i i}\left[M_{i i}^{-}\right]} \\
l & =0, \ldots, n_{i}^{+}-n_{i}^{-}, i \in \mathbb{X} .
\end{aligned}
$$

Since $\pi_{i}(\varepsilon)>0, i \in \mathbb{X}, \varepsilon \in\left(0, \varepsilon_{0}\right]$, the asymptotic expansions (107) are pivotal, i.e., coefficients,

$$
c_{i}\left[n_{i}^{-}\right]=b_{i}\left[m_{i}^{-}\right] / B_{i i}\left[M_{i i}^{-}\right]>0, i \in \mathbb{X} .
$$

By the definition, $e_{i}(\varepsilon) \leq E_{i i}(\varepsilon), i \in \mathbb{X}, \varepsilon \in\left(0, \varepsilon_{0}\right]$. This implies that parameters $M_{i}^{-} \leq m_{i}^{-}, i \in \mathbb{X}$ and thus, parameters

$$
n_{i}^{-} \geq 0, i \in \mathbb{X}
$$

Moreover, since $\sum_{i \in \mathbb{X}} \pi_{i}(\varepsilon)=1$, for every $\varepsilon \in\left(0, \varepsilon_{0}\right]$, the parameters $n_{i}^{ \pm}, i \in \mathbb{X}$ and coefficients $c_{i}[l], l=n_{i}^{-}, \ldots, n_{i}^{+}, i \in \mathbb{X}$ satisfies the following relations,

$$
n^{-}=\min _{i \in \mathbb{X}} n_{i}^{-}=0
$$

and

$$
c[l]=\sum_{i \in \mathbb{X}} c_{i}[l]= \begin{cases}1 & \text { for } l=0, \\ 0 & \text { for } 0<l \leq n^{+}=\min _{i \in \mathbb{X}} n_{i}^{+} .\end{cases}
$$

Let us introduce sets,

$$
\mathbb{X}_{0}=\left\{i \in \mathbb{X}: n_{i}^{-}=0\right\}
$$

By the above remarks, the following relation takes place,

$$
\pi_{i}(0)=\lim _{\varepsilon \rightarrow 0} \pi_{i}(\varepsilon)=\left\{\begin{array}{cl}
c_{i}[0]>0 & \text { if } i \in \mathbb{X}_{0}, \\
0 & \text { if } i \notin \mathbb{X}_{0}
\end{array}\right.
$$

Theorem 8. Let conditions A - F hold for semi-Markov processes $\eta^{(\varepsilon)}(t)$. Then, the $\left(n_{i}^{-}, n_{i}^{+}\right)$-expansions (110), for the stationary probabilities $\pi_{i}(\varepsilon), i \in \mathbb{X}$ given by Algorithm 9, can be written down. This expansion is invariant with respect to the choice of permutation $\left\langle r_{1}, \ldots, r_{N-1}, i\right\rangle$ of sequence $\langle 1, \ldots, N\rangle$, in the above algorithm. Relations (113) - (117) hold for these expansions. 
Algorithm 10. This is an algorithm for computing upper bounds for remainders in asymptotic expansions for stationary probabilities of perturbed semi-Markov processes.

10.1. Conditions $\mathbf{A}, \mathbf{B}^{\prime}, \mathbf{C}-\mathbf{E}, \mathbf{F}^{\prime}$ and the proposition (i) (the multiple summation rule) of Lemma 6 imply that the $\left(m_{i}^{-}, m_{i}^{+}\right)$-expansions for expectations $e_{i}(\varepsilon), i \in \mathbb{X}$ are $\left(m_{i}^{-}, m_{i}^{+}, \dot{\delta}_{i}, \dot{G}_{i}, \dot{\varepsilon}_{i}\right)$-expansions, with parameters $\dot{\delta}_{i}, \dot{G}_{i}, \dot{\varepsilon}_{i}, i \in \mathbb{X}$ given by the following formulas,

$$
\begin{aligned}
\dot{\delta}_{i} & =\min _{j \in \mathbb{Y}_{i}, m_{i j}^{+}=m_{i}^{+}} \dot{\delta}_{i j}, \\
\dot{G}_{i} & =\sum_{j \in \mathbb{Y}_{i}}\left(\dot{G}_{i j} \dot{\varepsilon}_{i}^{m_{i j}^{+}+\dot{\delta}_{i j}-m_{i}^{+}-\dot{\delta}_{i}}+\sum_{m_{i}^{+}<j \leq m_{i j}^{+}}\left|b_{i j}\right| \dot{\varepsilon}_{i}^{j-m_{i}^{+}-\dot{\delta}_{i}}\right), \\
\dot{\varepsilon}_{i} & =\min _{j \in \mathbb{Y}_{i}} \dot{\varepsilon}_{i j} .
\end{aligned}
$$

10.2. Conditions $\mathbf{A}, \mathbf{B}^{\prime}, \mathbf{C}-\mathbf{E}, \mathbf{F}^{\prime}$ and the propositions (iv) (the reciprocal rule) and ( $\mathbf{v})$ (the division rule) of Lemma 6 imply that the $\left(n_{i}^{-}, n_{i}^{+}\right)$expansions for expectations $\pi_{i}(\varepsilon), i \in \mathbb{X}$ are $\left(n_{i}^{-}, n_{i}^{+}, \delta_{i}^{*}, G_{i}^{*}, \varepsilon_{i}^{*}\right)$-expansions, with parameters $\delta_{i}^{*}, G_{i}^{*}, \varepsilon_{i}^{*}, i \in \mathbb{X}$ given by the following formulas,

$$
\begin{aligned}
& \delta_{i}^{*}= \begin{cases}\dot{\delta}_{i} & \text { if } n_{i}^{+}=m_{i}^{+}-M_{i i}^{-}<n_{i}^{-}+M_{i i}^{+}-2 M_{i i}^{-}, \\
\dot{\delta}_{i} \wedge \delta_{i i}^{\circ} & \text { if } n_{i}^{+}=m_{i}^{+}-M_{i i}^{-}<n_{i}^{-}+M_{i i}^{+}-2 M_{i i}^{-}, \\
\delta_{B} & \text { if } n_{i}^{+}=n_{i}^{-}+M_{i i}^{+}-2 M_{i i}^{-}<m_{i}^{+}-M_{i i}^{-},\end{cases} \\
& G_{i}^{*}=\left(\frac{B_{i i}\left[M_{i i}^{-}\right]}{2}\right)^{-1}\left(\sum_{m_{i}^{+} \wedge\left(m_{i}^{-}+M_{i i}^{+}-M_{i i}^{-}\right)<l \leq m_{i}^{+}}\left|b_{i}[l]\right|\left(\varepsilon_{i}^{*}\right)^{l-n_{i}^{+}-M_{i i}^{-}-\delta_{i}^{*}}\right. \\
& +\sum_{m_{i}^{+} \wedge\left(m_{i}^{-}+M_{i i}^{+}-M_{i i}^{-}\right)<l+k, m_{i}^{-} \leq l \leq m_{i}^{+}, M_{i i}^{-} \leq k \leq M_{i i}^{+}}\left|b_{i}[l]\right|\left|c_{i}[k]\right|\left(\varepsilon_{i}^{*}\right)^{l+k-n_{i}^{+}-M_{i i}^{-}-\delta_{i}^{*}} \\
& +\dot{G}_{i}\left(\varepsilon_{i}^{*}\right)^{m_{i}^{+}+\dot{\delta}_{i}-n_{i}^{+}-M_{i i}^{-}-\delta_{i}^{*}} \\
& \left.+G_{i i}^{\circ} \sum_{n_{i}^{-} \leq k \leq n_{i}^{+}}\left|c_{i}[k]\right|\left(\varepsilon_{i}^{*}\right)^{k+M_{i i}^{+}+\delta_{i}^{\circ}-n_{i}^{+}-M_{i i}^{-}-\delta_{i}^{*}}\right), \\
& \varepsilon_{i}^{*}=\dot{\varepsilon}_{i} \wedge \varepsilon_{i}^{\circ} \wedge \begin{cases}\frac{B_{i i}\left[M_{i i}^{-}\right]}{2}\left(\sum_{M_{i i}^{-}<l \leq M_{i i}^{+}}\left|B_{i i}[l]\right|\left(\varepsilon_{i}^{\circ}\right)^{l-M_{i i}^{-}-1}\right. & \\
\left.+G_{i i}^{\circ}\left(\varepsilon_{i}^{\circ}\right)^{M_{i i}^{+}+\delta_{i}^{\circ}-M_{i i}^{-}-1}\right)^{-1} & \text { if } M_{i i}^{-}<M_{i i}^{+}, \\
\left(\frac{B_{i i}\left[M_{i i}^{-}\right]}{2 G_{i}^{\circ}}\right)^{\frac{1}{\delta_{i}^{\circ}}} & \text { if } M_{i i}^{-}=M_{i i}^{+} .\end{cases}
\end{aligned}
$$


Theorem 9. Let conditions $\mathbf{A}, \mathbf{B}^{\prime}, \mathbf{C}-\mathbf{E}, \mathbf{F}^{\prime}$ hold for semi-Markov processes $\eta^{(\varepsilon)}(t)$. Then, the $\left(n_{i}^{-}, n_{i}^{+}, \delta_{i}^{*}, G_{i}^{*}, \varepsilon_{i}^{*}\right)$-expansions (110) for the stationary probabilities $\pi_{i}(\varepsilon), i \in \mathbb{X}$ given by Algorithms 9 and 10 can be written down. The inequalities $\delta_{i}^{*} \geq \delta^{\circ}, i \in \mathbb{X}$ hold, where parameter $\delta^{\circ}$ is given in relation (105).

7.3. Laurent asymptotic expansions for expectations of hitting times. Algorithms presented above yields the Laurent asymptotic expansions for expectations of hitting times $E_{i j}(\varepsilon), i, j \in \mathbb{X}$. Indeed, let choose two states $i, j \in \mathbb{X}$ and a chain of states $\bar{r}_{N-2}=\left(r_{1}, \ldots, r_{N-2}\right), r_{1}, \ldots, r_{N-2} \neq i, j$.

According the Theorem 1 and Algorithm 8 the expectations $E_{i j}(\varepsilon)$ coincides for the initial semi-Markov process $\eta^{(\varepsilon)}(t)$ and the semi-Markov process $\bar{r}_{N-2} \eta^{(\varepsilon)}(t)$. The semi-Markov process $\bar{r}_{N-2} \eta^{(\varepsilon)}(t)$ has a two-points phase space $\bar{r}_{N-2} \mathbb{X}=\{i, j\}$. The expectations of hitting times $E_{i^{\prime} j^{\prime}}(\varepsilon), i^{\prime} \in\{i, j\}$ can be found by solving, for every $j^{\prime} \in\{i, j\}$, the system of (two, in this case) linear equations (777) that yields the following formulas, for every $j^{\prime} \in\{i, j\}$,

$$
\left\{\begin{array}{l}
E_{i^{\prime} j^{\prime}}(\varepsilon)=\bar{r}_{N-2} e_{i^{\prime}}(\varepsilon) \cdot \frac{1}{\bar{r}_{N-2} p_{i^{\prime} j^{\prime}}(\varepsilon)}, \\
E_{j^{\prime} j^{\prime}}(\varepsilon)=\bar{r}_{N-2} e_{j^{\prime}}(\varepsilon)+\bar{r}_{N-2} e_{i^{\prime}}(\varepsilon) \cdot \frac{\bar{r}_{N-2} p_{j^{\prime} i^{\prime}}(\varepsilon)}{\bar{r}_{N-2} p_{i^{\prime} j^{\prime}}(\varepsilon)},
\end{array}\right.
$$

where $i^{\prime} \neq j^{\prime}$ in both equations in (120) and,

$$
\bar{r}_{N-2} e_{i^{\prime}}(\varepsilon)={ }_{\bar{r}_{N-2}} e_{i^{\prime} i}(\varepsilon)+{ }_{\bar{r}_{N-2}} e_{i^{\prime} j}(\varepsilon), i^{\prime} \in\{i, j\} .
$$

The corresponding asymptotic expansions for $E_{i j}(\varepsilon)$ can be constructing by using the asymptotic expansions for transition probabilities $p_{j^{\prime} i^{\prime}}(\varepsilon)$ and expectations $\bar{r}_{N-2} e_{i^{\prime}}(\varepsilon)$ given in Algorithms 7 and 8 and the operational rules for Laurent asymptotic expansions presented in Lemmas $1-9$.

\section{Future studies and bibliographical remarks}

In this section, we present some directions for future studies and short bibliographical remarks concerned works in the area.

8.1. Directions for future studies. The method of sequential reduction of a phase space presented in the paper can also be applied for getting asymptotic expansions for high order power and exponential moments of hitting times, for nonlinearly perturbed semi-Markov processes. 
In the present paper, we consider the model, where the pre-limiting perturbed semi-Markov processes have a phase space which is one class of communicative states, while the limiting unperturbed semi-Markov process has a phase space which consists of one or several classes of communicative states and possibly a class of transient states. However, the method of sequential reduction of the phase space can also be applied to nonlinearly perturbed semi-Markov processes with absorption and, therefore, to the model, where the pre-limiting semi-Markov processes also have a phase space, which consists of several classes of communicative states and a class of transient states.

We are quite sure that combination of results in the above two directions with the methods of asymptotic analysis for nonlinearly perturbed regenerative processes developed in Silvestrov (1995, 2007, 2010) and Gyllenberg and Silvestrov $(1998,1999 a$ a, 2000a, 2008) will make it possible to expand results concerned asymptotic expansions for quasi-stationary distributions and other characteristics for nonlinearly perturbed semi-Markov processes with absorption, where the limiting semi-Markov process has a phase space which consists of one class of communicative states and a class of transient states, to a general case, where the limiting semi-Markov process has a phase space, which consists of several classes of communicative states and a class of transient states.

The problems of aggregation of steps in the time-space screening procedures for semi-Markov processes, tracing pivotal orders for different groups of states as well as getting explicit matrix formulas, for coefficients and parameters of upper bounds for remainders in the corresponding asymptotic expansions for stationary distributions and moments of hitting times, do require additional studies. It can be expected that such formulas can be obtained, for example, for birth-death type semi-Markov processes, for which the proposed algorithms of reduction of a phase space preserve the birth-death structure for reduced semi-Markov processes.

We are going to present results concerned Laurent asymptotic expansions for power and exponential moments of hitting times, quasi-stationary distributions and explicit formulas for coefficients and parameters of upper bounds for remainders for some specific classes of semi-Markov models, as well as applications to some models of population genetics, information networks and queuing systems, in future publications.

8.2. Bibliographical remarks. Note first of all that the model of perturbed discrete time Markov chains, at least, in the most difficult case of so-called singularly perturbed Markov chains and semi-Markov processes with absorption and asymptotically uncoupled phase spaces, attracted attention of researchers in the mid of the 20th century.

(1) The first works related to asymptotical problems for the above models are Meshalkin (1958), Simon, and Ando (1961), Hanen (1963a, b, c, 1964), Seneta 
(1967, 1968a, b, 1973), Schweitzer (1968), Korolyuk (1969), Silvestrov (1969, 1970, 1971a, b, 1972a, b, 1974), Anisimov (1970, 1971a, b, 1973a, b), Korolyuk and Turbin (1970, 1972), Gusak and Korolyuk (1971), Turbin (1971, 1972), Korolyuk, Penev and Turbin (1972), Kovalenko (1973, 1975), Poliščuk and Turbin (1973), Korolyuk, Brodi and Turbin (1974), Pervozvanskiı̌ and Smirnov (1974), Courtois (1975) and Gaŭtsgori and Pervozvanskiǔ (1975).

(2) Convergence results, for distributions and moments of hitting times, eigenvalues, eigenvectors, stationary and quasi-stationary distributions, Perron roots, coefficients of ergodicity, etc. have been studied in works by Meshalkin (1958), Hanen, (1963a, b, c, 1964), Seneta (1967, 1968a, b, 1973, 2006), Schweitzer (1968, 1984), Korolyuk (1969, 1989), Silvestrov (1969, 1970, 1971a, 1972a, 1974, 1976, 1978, 1979a, b, 1981, 2000), Anisimov (1970, 1971a, b, 1973a, b, 1980, 1986, 1988, 2008), Korolyuk and Turbin (1970, 1972, 1976, 1978), Gusak and Korolyuk (1971), Turbin (1971), Korolyuk, Penev and Turbin (1972), Kovalenko (1973, 1975), Korolyuk, Brodi and Turbin (1974), Gaitsgori and Pervozvanskiy (1975, 1983), Allen, Anderssen and Seneta (1977), Kaplan (1979, 1980), Korolyuk, Turbin and Tomusjak (1979), Shurenkov (1980a, b), Anisimov and Chernyak (1982), Anisimov, Voŭna and Lebedev (1983), Coderch, Willsky, Sastry and Castañon (1983), Korolyuk, D. and Silvestrov (1983, 1984), Stewart (1983, 1984), Koury, McAllister and Stewart (1984), McAllister, Stewart and Stewart, W. (1984), Cao and Stewart (1985), Kartashov (1986, 1987, 1996b), Burnley (1987), Gibson and Seneta (1987), Haviv (1987), Haviv, Ritov and Rothblum (1987), Rohlichek (1987), Rohlicek and Willsky (1988a, b), Silvestrov and Velikii (1988), Hunter (1991a), Latouche (1991), Pollett and Stewart (1994), Hoppensteadt, Salehi and Skorokhod (1996a), Kalashnikov (1997), Korolyuk and Limnios (1998, 1999, 2000, 2002, 2004a, b, 2005), Marek and Mayer (1998), Yin and Zhang (1998, 2005, 2013), Craven (2003), Hernández-Lerma and Lasserre (2003), Yin, Zhang and Badowski (2003), Silvestrov and Drozdenko (2005, 2006), Drozdenko (2007a, b, 2009), Barbour and Pollett (2010, 2012) and Meyer (2015).

(3) Rates of convergence, errors of approximation, sensitivity and related stability theorems for Markov chains and related models of stochastic processes have been studied in works by Schweitzer (1968, 1986, 1987, 1991), Silvestrov (1969, 1971b, 1972b), Seneta (1973, 1984, 1988a, b, 1991, 1993, 2006), Courtois (1975, 1982), Gaĭtsgori and Pervozvanskiy (1975, 1983), Kovalenko (1975), Kalashnikov (1978, 1997a, 1997b), Louchard and Latouche (1978, 1982 1990), Berman and Plemmons (1979, 1994), Meyer (1980, 1994), Kalashnikov and Anichkin (1981), Bobrova (1983), Stewart (1983, 1984a, 1990, 1991, 1993a, b, 1998, 2001, 2003), Courtois and Semal (1984a, b, c, 1991), Haviv and Rothblum (1984), Haviv and Van der Heyden (1984), Koury, McAllister and Stewart (1984), McAllister, Stewart, G. and Stewart, W. (1984), Funderlic and Meyer (1985), Kartashov (1985a, b, 
c, 1986, 1987, 1988, 1996a, 1996b, 2000, 2005), Vantilborgh (1985), Haviv (1986, 1992, 2006), Haviv and Ritov (1986, 1994), Rohlichek (1987), Rohlicek and Willsky (1988a, b), Stewart and Sun (1990), Hunter (1991a, b, 2005, 2014), Stewart and Zhang (1991), Hassin and Haviv (1992, 1999), Barlow (1993), Meyn and Tweedie (1993, 2009), Lasserre (1994), Pollett and Stewart (1994), Stewart, G., Stewart, W. and McAllister (1994), Borovkov (1998), Yin and Zhang (1998, 2003, 2005, 2013), Li, Yin, G., Yin, K. and Zhang (1999), Craven (2003), Kontoyiannis and Meyn (2003), Zhang and Yin (2004), Guo (2006) and Sirl, Zhang and Pollett (2007).

(4) Asymptotic expansions for distributions of hitting times, moments of hitting times, resolvents, eigenvalues, eigenvectors, stationary and quasi-stationary distributions, Perron roots, etc., have been studied in works by Turbin (1972), Poliščuk and Turbin (1973), Koroljuk, Brodi and Turbin (1974), Pervozvanskiı̌ and Smirnov (1974), Courtois and Louchard (1976), Korolyuk and Turbin (1976, 1978), Courtois (1977), Latouche and Louchard (1978), Kokotović, Phillips and Javid (1980), Korolyuk, Penev and Turbin (1981), Phillips and Kokotović (1981), Delebecque (1983), Abadov (1984), Kartashov (1985d, 1996b), Haviv (1986), Korolyuk (1989), Stewart and Sun (1990), Silvestrov and Abadov (1991, 1993), Haviv, Ritov and Rothblum (1992), Haviv and Ritov (1993), Schweitzer and Stewart (1993), Silvestrov (1995, 2007, 2010), Englund and Silvestrov (1997), Gyllenberg and Silvestrov (1998, 1999a, 2000a, 2008), Korolyuk and Limnios (1998, 1999, 2000, 2002, 2004a, b, 2005), Stewart (1998, 2001), Yin and Zhang (1998, 2003, 2005, 2013), Avrachenkov (1999, 2000), Avrachenkov and Lasserre (1999), Korolyuk, V.S. and Korolyuk, V.V. (1999), Englund (2000, 2001), Yin, G., Zhang, Yang and Yin, K. (2001), Avrachenkov and Haviv (2003, 2004), Craven (2003) and Avrachenkov, Filar and Howlett (2013).

(5) Asymptotic expansions for other characteristics of Markov type processes are presented in works by Nagaev $(1957,1961)$, Leadbetter (1963), Poliščuk and Turbin(1973), Quadrat (1983), Abadov (1984), Silvestrov and Abadov (1984, 1991, 1993), Stewart and Sun (1990), Kartashov (1996b, 2013), Khasminskii, Yin and Zhang (1996a, b), Wentzell (1996, 1999), Yin and Zhang (1996b), Cao (1998), Gyllenberg and Silvestrov, (1998, 1999a, 2000a, 2000c, 2008), Fuh and Lai (2001), Kontoyiannis and Meyn (2003), Fuh (2004, 2007), Samoulenko (2006a, b), Silvestrov (1995, 2007, 2010), Ni (2010a, b, 2011, 2012, 2014). Ni, Silvestrov and Malyarenko (2008), Albeverio, Koroliuk and Samoilenko (2009), Petersson (2013a, b, 2014), Avrachenkov, Filar and Howlett (2013) and Silvestrov and Petersson (2013), Silvestrov D. and Silvestrov, S. (2015).

(6) We would like esspesially to mention books including problems on perturbed Markov chains, semi-Markov processes and related problems. These are Seneta (1973, 2006), Silvestrov (1974), Korolyuk and Turbin $(1976,1978)$, Courtois (1977), Kalashnikov (1978, 1997b), Anisimov (1988, 2008), Stewart and Sun 
(1990), Korolyuk and Swishchuk (1992), Meyn and Tweedie (1993, 2009), Kartashov (1996b), Borovkov (1998), Stewart (1998, 2001),Yin and Zhang (1998, 2005, 2013), Korolyuk, V.S. and Korolyuk, V.V. (1999), Bini, Latouche and Meini (2005), Koroliuk and Limnios (2005), Gyllenberg and Silvestrov (2008), and Avrachenkov, Filar and Howlett (2013).

(7) General results of perturbation theory of matrices and linear operators are presented in works by Vishik and Lyusternik (1960), Stewart (1969, 1973, 1979, 1984b, 1998, 2001), Plotkin and Turbin (1971, 1975), Korolyuk and Turbin (1976, 1978), Berman and Plemmons (1979, 1994), Wentzell and Freidlin (1979), Haviv (1988), Meyer and Stewart (1988), Bielecki and Stettner (1989), Delebecque (1990), Stewart and Sun (1990), Hunter (1991b, 2014), Haviv and Ritov (1994), Lasserre (1994), Hoppensteadt, Salehi and Skorokhod (1997), Avrachenkov (1999), Korolyuk, V.S. and Korolyuk, V.V. (1999), Li and Stewart (2000), Avrachenkov, Haviv and Howlett (2001), Howlett and Avrachenkov (2001), Howlett, Pearce and Torokhti (2003), Torokhti, Howlett and Pearce (2003), Verhulst ( 2005), Kartashov (1996b), Avrachenkov, Pearce and Ejov (2009), Howlett, Albrecht and Pearce (2010, 2014), Albrecht, Howlett and Pearce (2011), and Avrachenkov and Lasserre (2013). In particular, we would like to mention some books, which contains materials on general perturbation matrix and operator theory. These are Erdélyi (1956), Kato (1966, 2013), Cole (1968), Korolyuk and Turbin (1976, 1978), Wentzell and Freidlin (1979), Kevorkian and Cole (1981, 1985, 1996, 2011), Baumgärtel (1985), Stewart (1998, 2001), Korolyuk, V.S. and Korolyuk, V.V. (1999), Konstantinov, Gu, Mehrmann and Petkov (2003), Verhulst (2005), Gyllenberg and Silvestrov (2008), and Avrachenkov, Filar and Howlett (2013).

(8) Applications of results on perturbed Markov type processes to the control theory, decision processes, Internet, queuing theory, mathematical genetics, population dynamics and epidemic models, insurance and financial mathematics are presented in works by Simon and Ando (1961), Courtois (1977), Kalashnikov (1978, 1997b), Delebecque and Quadrat (1981), Quadrat (1983), Schweitzer (1984), Anisimov, Zakusilo and Donchenko (1987), Latouche (1988), Pervozvanskii and Gaitsgori (1988), Meyer (1989), Ho and Cao (1991), Gyllenberg and Silvestrov (1994, 1999b, 2000b, 2008, 2014), Pollett and Stewart (1994), Abbad and Filar (1995), Hoppensteadt, Salehi and Skorokhod (1996b), Kovalenko, Kuznetsov, and Pegg (1997), Borovkov (1998), Yin and Zhang (1998, 2005, 2013), Englund (1999a, b), Yin, G., Zhang, Yang and Yin, K. (2001), Avrachenkov, Filar and Haviv (2002), Altman, Avrachenkov and Núñez-Queija (2004), Langville and Meyer (2006), Avrachenkov, Litvak, and Son Pham (2007, 2008), Andersson and Silvestrov, S. (2008), Anisimov (2008), Konstantinov and Petkov (2008), Avrachenkov, Borkar and Nemirovsky (2010), Barbour and Pollett (2010, 2012), Hössjer (2011, 2014), Engström and Silvestrov, S. (2014), Hössjer and Ryman (2014), Ni (2014), 
Petersson (2014), Silvestrov $(2014,2015)$. In particular, we would like to mention some books in this area that are Kalashnikov (1978, 1997b), Anisimov, Zakusilo and Donchenko (1987), Pervozvanskiı and Gaitsgori (1988), Kovalenko, Kuznetsov, and Pegg (1997), (1998), Anisimov (2008), Gyllenberg and Silvestrov (2008), Avrachenkov, Filar and Howlett (2013), and Silvestrov (2014, 2015).

(9) Exact and related approximative computational methods for stationary and quasi-stationary distributions of Markov chains and semi-Markov processes and related problems are presented in works by Romanovskiu (1949), Feller (1950, 1968), Kemeny and Snell (1960), Golub and Seneta (1973), Seneta (1973, 2006), Paige, Styan and Wachter (1975), Silvestrov (1980a, b, 1996), Chatelin and Miranker (1984), Harrod and Plemmons (1984), Schweitzer (1984, 1991), Grassman, Taksar and Heyman (1985), Schweitzer, Puterman and Kindle (1985), Sheskin (1985), Hunter (1986, 1991a), Schweitzer and Kindle (1986), Feinberg and Chiu (1987), Haviv (1987, 1992), Haviv, Ritov and Rothblum (1987), Latouche and Ramaswami (1987, 1999), Sumita and Reiders (1988), Mattingly and Meyer (1991), Stewart, W. (1994), Kim and Smith (1995), Stewart (1998, 2001), Kartashov (2000), Meyer (2000), Häggström (2002), Bini, Latouche and Meini (2005), Golub and Van Loan (2013), Silvestrov, Manca and Silvestrova (2014). In particular, we would like to mention some related books that are Romanovskiı̌ (1949), Feller (1950, 1968), Kemeny and Snell (1960), Golub and Seneta (1973), Seneta (1973, 2006), Berman and Plemmons (1979, 1994), Silvestrov (1980a), Meyer (2000), Häggström (2002), Bini, Latouche and Meini (2005), Meyn and Tweedie (1993, 2009), Hernández-Lerma and Lasserre, (2003), Gyllenberg and Silvestrov (2008), and Collet, Martínez and San Martín (2013).

\section{References}

[1] Abadov, Z.A. (1984). Asymptotical Expansions with Explicit Estimation of Constants for Exponential Moments of Sums of Random Variables Defined on a Markov Chain and their Applications to Limit Theorems for First Hitting Times. Candidate of Science dissertation, Kiev State University.

[2] Abbad, M., Filar, J.A. (1995). Algorithms for singularly perturbed Markov control problems: A survey. In: Leondes, C.T. (Ed). Techniques in Discrete-Time Stochastic Control Systems. Control and Dynamic Systems, 73, Academic Press, New York, 257-289.

[3] Albeverio, S., Koroliuk, V.S., Samoilenko, I.V. (2009). Asymptotic expansion of semi-Markov random evolutions. Stochastics, 81, no. 5, 477-502. 
[4] Albrecht, A.R., Howlett, P.G., Pearce, C.E.M. (2011). Necessary and sufficient conditions for the inversion of linearly-perturbed bounded linear operators on Banach space using Laurent series. J. Math. Anal. Appl., 383, no. 1, 95-110.

[5] Albrecht, A.R., Howlett, P.G., Pearce, C.E.M. (2014). The fundamental equations for inversion of operator pencils on Banach space. J. Math. Anal. Appl., 413, no. 1, 411-421.

[6] Allen, B., Anderssen, R.S., Seneta, E. (1977). Computation of stationary measures for infinite Markov chains. In: Neuts, M.F. (Ed). Algorithmic Methods in Probability. Studies in the Management Sciences, 7. North-Holland, Amsterdam, 13-23.

[7] Altman, E., Avrachenkov, K.E., Núñez-Queija, R. (2004). Perturbation analysis for denumerable Markov chains with application to queueing models. Adv. Appl. Probab., 36, no. 3, 839-853.

[8] Andersson, F., Silvestrov, S. (2008). The mathematics of Internet search engines. Acta Appl. Math., 104, 211-242.

[9] Anisimov, V.V. (1970). Limit theorems for semi-Markov processes. I, II. Teor. Veroyatn. Mat. Stat., 2, I: 3-12; II: 1-21.

[10] Anisimov, V.V. (1971a). Limit theorems for sums of random variables on a Markov chain, connected with the exit from a set that forms a single class in the limit. Teor. Veroyatn. Mat. Stat., 4, 3-17 (English translation in Theory Probab. Math. Statist., 4, 1-13).

[11] Anisimov, V.V. (1971b). Limit theorems for sums of random variables that are given on a subset of states of a Markov chain up to the moment of exit, in a series scheme. Teor. Veroyatn. Mat. Stat., 4, 18-26 (English translation in Theory Probab. Math. Statist., 4, 15-22).

[12] Anisimov, V.V. (1973a). Limit theorems for sums of random variables that are given on a countable subset of the states of a Markov chain up to the first exit time. Teor. Veroyatn. Mat. Stat., 8, 3-13.

[13] Anisimov, V.V. (1973b). Asymptotical consolidation of states for random processes. Kibernetika, no. 3, 109-117.

[14] Anisimov, V.V. (1980). Limit theorems for processes admitting the asymptotical consolidation of states. Theor. Veroyatn. Mat. Stat., 22, 3-15 (English translation in Theory Probab. Math. Statist., 22, 1-13).

[15] Anisimov, V.V. (1986). Approximation of Markov processes that can be asymptotically lumped. Teor. Veroyatn. Mat. Stat., 34, 1-12 (English translation in Theory Probab. Math. Statist., 34, 1-11).

[16] Anisimov, V.V. (1988). Random Processes with Discrete Components. Vysshaya Shkola and Izdatel'stvo Kievskogo Universiteta, Kiev, 183 pp.

[17] Anisimov, V.V. (2008). Switching Processes in Queueing Models. Applied Stochastic Methods Series. ISTE, London and Wiley, Hoboken, NJ, 345 pp. 
[18] Anisimov, V.V., Chernyak, A.V. (1982). Limit theorems for certain rare functionals on Markov chains and semi-Markov processes. Teor. Veroyatn. Mat. Stat., 26, 3-8 (English translation in Theory Probab. Math. Statist., 26, 1-6).

[19] Anisimov, V.V., Voĭna, A.A., Lebedev, E.A. (1983). Asymptotic estimation of integral functionals and consolidation of stochastic systems. Vestnik Kiev. Univ., Model. Optim. Slozhn. Sist., no. 2, 41-50.

[20] Anisimov, V.V., Zakusilo, O.K., Donchenko, V.S. (1987). Elements of Queueing and Asymptotical Analysis of Systems. Lybid', Kiev, 248 pp.

[21] Avrachenkov, K.E. (1999). Analytic Perturbation Theory and Its Applications. PhD Thesis, University of South Australia, 1999.

[22] Avrachenkov, K.E. (2000). Singularly perturbed finite Markov chains with general ergodic structure. In: R. Boel, R., Stremersch, G. (Eds). Discrete Event Systems. Analysis and Control. Kluwer International Series in Engineering and Computer Science, 569, Kluwer, Boston, 429-432.

[23] Avrachenkov, K., Borkar, V., Nemirovsky, D. (2010). Quasi-stationary distributions as centrality measures for the giant strongly connected component of a reducible graph. J. Comput. Appl. Math., 234, no. 11, 3075-3090.

[24] Avrachenkov, K.E., Filar, J., Haviv, M. (2002). Singular perturbations of Markov chains and decision processes. In: Feinberg, E.A., Shwartz, A. (Eds). Handbook of Markov Decision Processes. Methods and Applications. International Series in Operations Research \& Management Science 40, Kluwer, Boston, 113-150.

[25] Avrachenkov, K.E., Filar, J.A., Howlett, P.G. (2013). Analytic Perturbation Theory and Its Applications. SIAM, Philadelphia, PA, xii+372 pp.

[26] Avrachenkov, K.E., Haviv, M. (2003). Perturbation of null spaces with application to the eigenvalue problem and generalized inverses. Linear Algebra Appl., 369, 1-25.

[27] Avrachenkov, K.E., Haviv, M. (2004). The first Laurent series coefficients for singularly perturbed stochastic matrices. Linear Algebra Appl., 386, 243-259.

[28] Avrachenkov, K.E., Haviv, M., Howlett, P.G. (2001). Inversion of analytic matrix functions that are singular at the origin. SIAM J. Matrix Anal. Appl., 22, no. 4, $1175-1189$.

[29] Avrachenkov, K.E., Lasserre, J.B. (1999). The fundamental matrix of singularly perturbed Markov chains. Adv. Appl. Probab., 31, no. 3, 679-697.

[30] Avrachenkov, K.E., Lasserre, J.B. (2013). Analytic perturbation of generalized inverses. Linear Algebra Appl., 438, no. 4, 1793-1813.

[31] Avrachenkov, K., Litvak, N., Son Pham, K. (2007). Distribution of PageRank mass among principle components of the web. In: Chung, F.R.K., Bonato, A. (Eds).Algorithms and Models for the Web-Graph. Lecture Notes in Computer Science, 4863, Springer, Berlin, 16-28.

[32] Avrachenkov, K., Litvak, N., Son Pham, K. (2008). A singular perturbation approach for choosing the PageRank damping factor. Internet Math., 5, no. 1-2, 47-69. 
[33] Barbour, A.D., Pollett, P.K. (2010). Total variation approximation for quasistationary distributions. J. Appl. Probab., 47, no. 4, 934-946.

[34] Barbour, A.D., Pollett, P.K. (2012). Total variation approximation for quasiequilibrium distributions, II. Stoch. Process. Appl., 122, no. 11, 3740-3756.

[35] Barlow, J. (1993). Perturbation results for nearly uncoupled Markov chains with applications to iterative methods. Numer. Math., 65, no. 1, 51-62.

[36] Baumgärtel, H. (1985). Analytic Perturbation Theory for Matrices and Operators. Operator Theory: Advances and Applications, 15, Birkhäuser, Basel, 427 pp.

[37] Berman, A., Plemmons, R.J. $(1979,1994)$. Nonnegative Matrices in the Mathematical Sciences. Revised reprint of the 1979 original. Classics in Applied Mathematics, 9, SIAM, Philadelphia, $\mathrm{xx}+340 \mathrm{pp}$.

[38] Bielecki, T., Stettner, Ł. (1989). On ergodic control problems for singularly perturbed Markov processes. Appl. Math. Optim., 20, no. 2, 131-161.

[39] Bini, D.A., Latouche, G., Meini, B. (2005). Numerical Methods for Structured Markov Chains. Numerical Mathematics and Scientific Computation. Oxford Science Publications. Oxford University Press, New York, 2005. xii+327 pp.

[40] Bobrova, A.F. (1983). Estimates of accuracy of an asymptotic consolidation of countable Markov chains. In Stability Problems for Stochastic Models. Trudy Seminara, VNIISI, Moscow, 16-24.

[41] Borovkov, A.A. (1998). Ergodicity and Stability of Stochastic Processes. Translation from the 1994 Russian original. Wiley Series in Probability and Statistics: Probability and Statistics. Wiley, Chichester, xxiv +585 pp.

[42] Burnley, C. (1987). Perturbation of Markov chains. Math. Mag., 60, no. 1, 21-30.

[43] Cao, X.R. (1998). The Malaurin series for performance functions of Markov chains, Adv. Appl. Prob., 30, 676-692.

[44] Cao, W.L., Stewart, W.J. (1985). Iterative aggregation/disaggregation techniques for nearly uncoupled Markov chains. J. Ass. Comp. Mach., 32, 702-719.

[45] Chatelin, F., Miranker, W.L. (1984). Aggregation/disaggregation for eigenvalue problems. SIAM J. Numer. Anal., 21, no. 3, 567-582.

[46] Coderch, M., Willsky, A.S., Sastry, S.S., Castañon, D.A. (1983). Hierarchical aggregation of singularly perturbed finite state Markov processes. Stochastics, 8, 259-289.

[47] Cole, J.D. (1968). Perturbation Methods in Applied Mathematics. Blaisdell, Waltham, Mass., vi+260 pp.

[48] Collet, P., Martínez, S., San Martín, J. (2013). Quasi-Stationary Distributions. Markov Chains, Diffusions and Dynamical Systems. Probability and its Applications, Springer, Heidelberg, xvi+280 pp.

[49] Courtois, P.J. (1975). Error analysis in nearly-completely decomposable stochastic systems. Econometrica, 43, no. 4, 691-709.

[50] Courtois, P.J. (1977). Decomposability: Queueing and Computer System Applications. ACM Monograph Series, Academic Press, New York, xiii+201 pp. 
[51] Courtois, P.J. (1982). Error minimization in decomposable stochastic models. In: Ralph L.D., Teunis J.O. (Eds). Applied ProbabilityComputer Science: the Interface, Vol. I. Progress in Computer Science, 2, Birkhäuser Boston, Boston, 189210.

[52] Courtois, P.J., Louchard, G. (1976). Approximation of eigen characteristics in nearlycompletely decomposable stochastic systems. Stoch. Process. Appl., 4, 283-296.

[53] Courtois, P.J., Semal, P. (1984a). Error bounds for the analysis by decomposition of non-negative matrices. In: Iazeolla, G., Courtois, P.J., Hordijk, A. (Eds). Mathematical Computer Performance and Reliability. North-Holland, Amsterdam, 209-224.

[54] Courtois, P.J., Semal, P. (1984b). Block decomposition and iteration in stochastic matrices. Philips J. Res., 39, no. 4-5, 178-194.

[55] Courtois, P.J., Semal, P. (1984c). Bounds for the positive eigenvectors of nonnegative matrices and for their approximations by decomposition. J. Assoc. Comput. Mach.. 31, no. 4, 804-825.

[56] Courtois, P.J., Semal, P. (1991). Bounds for transient characteristics of large or infinite Markov chains. In: Stewart, W.J. (Ed). Numerical Solution of Markov Chains. Probability: Pure and Applied, 8, Marcel Dekker, New York, 413-434.

[57] Craven, B.D. (2003). Perturbed Markov processes. Stoch. Models, 19, no. 2, 269-285.

[58] Delebecque, F. (1983). A reduction process for perturbed Markov chains. SIAM J. Appl. Math., 43, 325-350.

[59] Delebecque, F. (1990). On the resolvent approach to the spectral decomposition of a regular matrix pencil. Linear Algebra Appl., 129, 63-75.

[60] Delebecque, F., Quadrat, J.P. (1981). Optimal control of Markov chains admitting strong and weak interactions. Automatica, 17, no. 2, 281-296.

[61] Drozdenko, M. (2007a). Weak convergence of first-rare-event times for semi-Markov processes. I. Theory Stoch. Process., 13, no. 4, 29-63.

[62] Drozdenko, M. (2007b). Weak Convergence of First-Rare-Event Times for SemiMarkov Processes. Doctoral dissertation 49, Mälardalen University, Västerås.

[63] Drozdenko, M. (2009). Weak convergence of first-rare-event times for semi-Markov processes. II. Theory Stoch. Process., 15, no. 2, 99-118.

[64] Englund, E. (1999a). Perturbed renewal equations with application to M/M queueing systems. 1. Teor. Imovirn. Mat. Stat., 60, 31-37 (Also in Theory Probab. Math. Statist., 60, 35-42).

[65] Englund, E. (1999b). Perturbed renewal equations with application to M/M queueing systems. 2. Teor. Imovirn. Mat. Stat., 61, 21-32 (Also in Theory Probab. Math. Statist., 61, 21-32).

[66] Englund, E. (2000). Nonlinearly perturbed renewal equations with applications to a random walk. In: Silvestrov, D., Yadrenko, M., Olenko A., Zinchenko, N. (Eds). Proceedings of the Third International School on Applied Statistics, Financial and Actuarial Mathematics, Feodosiya, 2000. Theory Stoch. Process., 6(22), no. 3-4, 33-60. 
[67] Englund, E. (2001). Nonlinearly Perturbed Renewal Equations with Applications. Doctoral dissertation, Umeå University.

[68] Englund, E., Silvestrov, D.S. (1997). Mixed large deviation and ergodic theorems for regenerative processes with discrete time. In: Jagers, P., Kulldorff, G., Portenko, N., Silvestrov, D. (Eds). Proceedings of the Second Scandinavian-Ukrainian Conference in Mathematical Statistics, Vol. I, Umeå, 1997. Theory Stoch. Process., 3(19), no. $1-2,164-176$.

[69] Engström, C., Silvestrov, S. (2014). Generalisation of the damping factor in PageRank for weighted networks. In: Silvestrov, D., Martin-Löf, A. (Eds). Modern Problems in Insurance Mathematics. Chapter 19, EAA series, Springer, Cham, 313-334.

[70] Erdélyi, A. (1956). Asymptotic Expansions. Dover, New York, vi+108 pp.

[71] Feinberg, B.N., Chiu, S.S. (1987). A method to calculate steady-state distributions of large Markov chains by aggregating states. Oper. Res., 35, no. 2, 282-290.

[72] Feller, W. $(1950,1968)$. An Introduction to Probability Theory and Its Applications. Vol. I., Third edition, Wiley, New York, xviii+509 pp.

[73] Fuh, C.D. (2004). Uniform Markov renewal theory and ruin probabilities in Markov random walks. Ann. Appl. Probab., 14, no. 3, 1202-1241.

[74] Fuh, C.D. (2007). Asymptotic expansions on moments of the first ladder height in Markov random walks with small drift. Adv. Appl. Probab., 39, 826-852.

[75] Fuh, C.D., Lai, T.L. (2001). Asymptotic expansions in multidimensional Markov renewal theory and first passage times for Markov random walks. Adv. Appl. Probab., 33, 652-673.

[76] Funderlic, R.E., Meyer, C.D., Jr. (1985). Sensitivity of the stationary distribution vector for an ergodic Markov chain. Linear Algebra Appl., 76, 1-17.

[77] Gaŭtsgori, V.G., Pervozvanskiŭ, A.A. (1975). Aggregation of states in a Markov chain with weak interaction. Kibernetika, no. 3, 91-98 (English translation in Cybernetics, 11, (1976), no. 3, 441-450).

[78] Gaitsgori, V.G., Pervozvanskiy, A. A. (1983). Decomposition and aggregation in problems with a small parameter. Izv. Akad. Nauk SSSR, Tekhn. Kibernet., no. 1, 33-46 (English translation in Engrg. Cybernetics, 2, (1984), no. 1, 26-38).

[79] Gibson, D., Seneta, E. (1987). Augmented truncations of infinite stochastic matrices. J. Appl. Probab., 24, no. 3, 600-608.

[80] Golub, G.H., Seneta, E. (1973). Computation of the stationary distribution of an infinite Markov matrix. Bull. Austral. Math. Soc., 8, 333-341.

[81] Golub, G.H., Van Loan, C.F. (1983, 1989, 1996, 2013). Matrix Computations. Fourth edition, Johns Hopkins Studies in the Mathematical Sciences, Johns Hopkins University Press, Baltimore, MD, xiv+756 pp.

[82] Grassman, W.K., Taksar, M.I., Heyman, D.P. (1985). Regenerative analysis and steady state distributions for Markov chains. Oper. Res., 33, 1107-1116.

[83] Guo D.Z. (2006). On the sensitivity of the solution of nearly uncoupled Markov chains. SIAM J. Matrix Anal. Appl., 14, no. 4, 1112-1123. 
[84] Gusak, D.V., Korolyuk, V.S. (1971). Asymptotic behaviour of semi-Markov processes with a decomposable set of states. Teor. Veroyatn. Mat. Stat., 5, 43-50 (English translation in Theory Probab. Math. Statist., 5, 43-51).

[85] Gyllenberg, M., Silvestrov, D.S. (1994). Quasi-stationary distributions of a stochastic metapopulation model. J. Math. Biol., 33, 35-70.

[86] Gyllenberg, M., Silvestrov, D.S. (1998). Quasi-stationary phenomena in semi-Markov models. In: Janssen, J., Limnios, N. (Eds). Proceedings of the Second International Symposium on Semi-Markov Models: Theory and Applications, Compiègne, 1998, 87-93.

[87] Gyllenberg, M., Silvestrov, D.S. (1999a). Quasi-stationary phenomena for semiMarkov processes. In: Janssen, J., Limnios, N. (Eds). Semi-Markov Models and Applications. Kluwer, Dordrecht, 33-60.

[88] Gyllenberg, M., Silvestrov, D.S. (1999b). Cramér-Lundberg and diffusion approximations for nonlinearly perturbed risk processes including numerical computation of ruin probabilities. In: Silvestrov, D., Yadrenko, M., Borisenko, O., Zinchenko, N. (Eds). Proceedings of the Second International School on Actuarial and Financial Mathematics, Kiev, 1999. Theory Stoch. Process., 5(21), no. 1-2, 6-21.

[89] Gyllenberg, M., Silvestrov, D.S. (2000a). Nonlinearly perturbed regenerative processes and pseudo-stationary phenomena for stochastic systems. Stoch. Process. Appl., 86, 1-27.

[90] Gyllenberg, M., Silvestrov, D.S. (2000b). Cramér-Lundberg approximation for nonlinearly perturbed risk processes. Insur. Math. Econom., 26, 75-90.

[91] Gyllenberg, M., Silvestrov, D.S. (2000c). Nonlinearly perturbed regenerative processes and pseudo-stationary phenomena for stochastic systems. Stoch. Process. Appl., 86, 1-27.

[92] Gyllenberg, M., Silvestrov, D.S. (2008). Quasi-Stationary Phenomena in Nonlinearly Perturbed Stochastic Systems. De Gruyter Expositions in Mathematics, 44, Walter de Gruyter, Berlin, ix+579 pp.

[93] Häggström, O. (2002). Finite Markov Chains and Algorithmic Applications. London Mathematical Society Student Texts, 52, Cambridge University Press, 126 pp.

[94] Hanen, A. (1963a). Probème central limite dans le cas Markovien fini. La matrice limite n'a qu'une seule classe ergodique et pas d'état transitoire. C. R. Acad. Sci. Paris, 256, 68-70.

[95] Hanen, A. (1963b). Problème central limite dans le cas Markovien fini. II. La matrice limite a plusieurs classes ergodiques et pas d'états transitoires. C. R. Acad. Sci. Paris, 256, 362-364.

[96] Hanen, A. (1963c). Problème central limite dans le cas Markovien fini. Cas général. C. R. Acad. Sci. Paris, 256, 575-577.

[97] Hanen, A. (1963d). Théorèmes limites pour une suite de chaînes de Markov. Ann. Inst. H. Poincaré, 18, 197-301. 
[98] Harrod,W.J., Plemmons, R.J. (1984). Comparison of some direct methods for computing stationary distributions of Markov chains. SIAM J. Sci. Statist. Compul., 5, 453-469.

[99] Hassin, R., Haviv, M. (1992). Mean passage times and nearly uncoupled Markov chains. SIAM J. Disc. Math., 5, 386-397.

[100] Haviv, M. (1986). An approximation to the stationary distribution of a nearly completely decomposable Markov chain and its error analysis. SIAM J. Algebr. Discr. Meth., 7, no. 4, 589-593.

[101] Haviv, M. (1987). Aggregation/disaggregation methods for computing the stationary distribution of a Markov chain. SIAM J. Numer. Anal., 24, no. 4, 952-966.

[102] Haviv, M. (1988). Error bounds on an approximation to the dominant eigenvector of a nonnegative matrix. Linear Multilinear Algebra, 23, no. 2, 159-163.

[103] Haviv, M. (1992). An aggregation/disaggregation algorithm for computing the stationary distribution of a large Markov chain. Comm. Statist. Stoch. Models, 8, no. $3,565-575$.

[104] Haviv, M. (1999). On censored Markov chains, best augmentations and aggregation/disaggregation procedures. Aggregation and disaggregation in operations research. Comput. Oper. Res., 26, no. 10-11, 1125-1132.

[105] Haviv, M. (2006). More on RayleighRitz refinement technique for nearly uncoupled stochastic matrices. SIAM J. Matrix Anal. Appl., 10, no. 3, 287-293.

[106] Haviv, M., Ritov, Y. (1986). An approximation to the stationary distribution of a nearly completely decomposable Markov chain and its error bound. SIAM J. Algebr. Discr. Methods, 7, no. 4, 583-588.

[107] Haviv, M., Ritov, Y. (1993). On series expansions and stochastic matrices. SIAM J. Matrix Anal. Appl., 14, no. 3, 670-676.

[108] Haviv, M., Ritov, Y. (1994). Bounds on the error of an approximate invariant subspace for non-self-adjoint matrices. Numer. Math., 67, no. 4, 491-500.

[109] Haviv, M., Ritov, Y., Rothblum, U.G. (1987). Iterative methods for approximating the subdominant modulus of an eigenvalue of a nonnegative matrix. Linear Algebra Appl., 87, 61-75.

[110] Haviv, M., Ritov, Y., Rothblum, U.G. (1992). Taylor expansions of eigenvalues of perturbed matrices with applications to spectral radii of nonnegative matrices. Linear Algebra Appl., 168, 159-188.

[111] Haviv, M., Rothblum, U.G. (1984). Bounds on distances between eigenvalues. Linear Algebra Appl., 63, 101-118.

[112] Haviv, M., Van der Heyden, L. (1984). Perturbation bounds for the stationary probabilities of a finite Markov chain. Adv. Appl. Probab., 16, 804-818.

[113] Hernández-Lerma, O. Lasserre, J.B. (2003). Markov Chains and Invariant Probabilities. Progress in Mathematics, 211, Birkhäuser, Basel, 2003. xvi+205 pp. 
[114] Ho, Y.C., Cao, X.R. (1991). Perturbation Analysis of Discrete Event Dynamic Systems. The Springer International Series in Engineering and Computer Science, Springer, New York, 433 pp.

[115] Hoppensteadt, F., Salehi, H., Skorokhod, A. (1996a). On the asymptotic behavior of Markov chains with small random perturbations of transition probabilities. In: Gupta, A.K. (Ed). Multidimensional Statistical Analysis and Theory of Random Matrices: Proceedings of the Sixth Eugene Lukacs Symposium. Bowling Green, OH, 1996. VSP, Utrecht, 93-100.

[116] Hoppensteadt, F., Salehi, H., Skorokhod, A. (1996b). Markov chain with small random perturbations with applications to bacterial genetics. Random Oper. Stoch. Equat., 4, no. 3, 205-227.

[117] Hoppensteadt, F., Salehi, H., Skorokhod, A. (1997). Discrete time semigroup transformations with random perturbations. J. Dynam. Dif. Equat., 9, no. 3, 463-505.

[118] Hössjer, O. (2011). Coalescence theory for a general class of structured populations with fast migration. Adv. in Appl. Probab., 43, no. 4, 1027-1047.

[119] Hössjer, O. (2014). Spatial autocorrelation for subdivided populations with invariant migration schemes. Methodol. Comput. Appl. Probab., 16, no. 4, 777-810.

[120] Hössjer, O., Ryman, N. (2014). Quasi equilibrium, variance effective size and fixation index for populations with substructure. J. Math. Biol., 69, no. 5, 1057-1128.

[121] Howlett, P., Albrecht, A., Pearce, C. (2010). Laurent series for inversion of linearly perturbed bounded linear operators on Banach space. J. Math. Anal. Appl., 366, no. $1,112-123$.

[122] Howlett, P., Avrachenkov, K. (2001). Laurent series for the inversion of perturbed linear operators on Hilbert space. In: Barney M. Glover, B.M., Rubinov, A.M. (Eds). Optimization and Related Topics. Applied Optimisation, 47, Kluwer, Dordrecht, $325-342$.

[123] Howlett, P., Avrachenkov, K., Pearce, C., Ejov, V. (2009). Inversion of analytically perturbed linear operators that are singular at the origin. J. Math. Anal. Appl., 353, no. $1,68-84$.

[124] Howlett, P., Pearce, C., Torokhti, A. (2003). On nonlinear operator approximation with preassigned accuracy. J. Comput. Anal. Appl., 5, no. 3, 273-297.

[125] Hunter, J.J. (1986). Stationary distributions of perturbed Markov chains. Linear Algebra Appl., 82, 201-214.

[126] Hunter, J.J. (1991a). The computation of stationary distributions of Markov chains through perturbations. J. Appl. Math. Stochastic Anal., 4, no. 1, 29-46.

[127] Hunter, J.J. (1991b). A survey of generalized inverses and their use in applied probability. Math. Chronicle, 20, 13-26.

[128] Hunter, J.J. (2005). Stationary distributions and mean first passage times of perturbed Markov chains. Linear Algebra Appl.. 410, 217-243.

[129] Hunter, J.J. (2014). Generalized inverses of Markovian kernels in terms of properties of the Markov chain. Linear Algebra Appl., 447, 38-55. 
[130] Kalashnikov, V.V. (1978). Qualitative Analysis of the Behaviour of Complex Systems by the Method of Test Functions. Series in Theory and Methods of Systems Analysis, Nauka, Moscow, 247 pp.

[131] Kalashnikov, V.V. (1997a). Solution of the problem of approximating a denumerable Markov chain. Engrg. Cybernetics, no. 3, 92-95.

[132] Kalashnikov, V.V. (1997b). Geometric Sums: Bounds for Rare Events with Applications. Mathematics and its Applications, 413, Kluwer, Dordrecht, ix+265 pp.

[133] Kalashnikov, V.V., Anichkin, S.A. (1981). Continuity of random sequences and approximation of Markov chains. Adv. Appl. Probab., 13, no. 2, 402-414.

[134] Kaplan, E.I. (1979). Limit theorems for exit times of random sequences with mixing. Teor. Veroyatn. Mat. Stat., 21, 53-59 (English translation in Theory Probab. Math. Statist., 21, 59-65)

[135] Kaplan, E.I. (1980). Limit Theorems for Sum of Switching Random Variables with an Arbitrary Phase Space of Switching Component. Candidate of Science dissertation, Kiev State University.

[136] Kartashov, N.V. (1985a). Inequalities in stability and ergodicity theorems for Markov chains with a general phase space. I. Teor. Veroyatn. Primen., 30, 230240 (English translation in Theory Probab. Appl., 30, 247-259).

[137] Kartashov, N.V. (1985b). Inequalities in stability and ergodicity theorems for Markov chains with a general phase space. II. Teor. Veroyatn. Primen., 30, 478485 (English translation in Theory Probab. Appl., 30, 507-515).

[138] Kartashov, N.V. (1985c). Asymptotic representations in an ergodic theorem for general Markov chains and their applications. Teor. Veroyatn. Mat. Stat., 32, 113-121 (English translation in Theory Probab. Math. Statist., 32, 131-139).

[139] Kartashov, N.V. (1985d). Asymptotic expansions and inequalities in stability theorems for general Markov chains under relatively bounded perturbations. In: Stability Problems for Stochastic Models, Varna, 1985. VNIISI, Moscow, 75-85 (English translation in J. Soviet Math., 40, no. 4, 509-518).

[140] Kartashov, N.V. (1986). Inequalities in theorems of consolidation of Markov chains. Theor. Veroyatn. Mat. Stat., 34, 62-73 (English translation in Theory Probab. Math. Statist., 34, 67-80)

[141] Kartashov, N.V. (1987). Estimates for the geometric asymptotics of Markov times on homogeneous chains. Teor. Veroyatn. Mat. Stat., 37, 66-77 (English translation in Theory Probab. Math. Statist., 37, 75-88).

[142] Kartashov, M.V. (1996a). Computation and estimation of the exponential ergodicity exponent for general Markov processes and chains with recurrent kernels. Teor. Imovirn. Mat. Stat., 54, 47-57 (English translation in Theory Probab. Math. Statist., $\mathbf{5 4}, 49-60)$.

[143] Kartashov, M.V. (1996b). Strong Stable Markov Chains. VSP, Utrecht and TBiMC, Kiev, 138 pp. 
[144] Kartashov, M.V. (2000). Calculation of the spectral ergodicity exponent for the birth and death process. Ukr. Mat. Zh., 52, 889-897 (English translation in Ukr. Math. $J ., \mathbf{5 2}, 1018-1028)$.

[145] Kartashov, M.V. (2005). Ergodicity and stability of quasihomogeneous Markov semigroups of operators. Teor. Imovirn. Mat. Stat., 72, 54-62 (English translation in Theory Probab. Math. Statist., 72, 59-68).

[146] Kartashov, M.V. (2013). Quantitative and qualitative limits for exponential asymptotics of hitting times for birth-and-death chains in a scheme of series. Teor. Imovirn. Mat. Stat., 89, 40-50 (English translation in Theory Probab. Math. Statist, 89, (2014), 45-56).

[147] Kato, T. (1966, 2013). Perturbation Theory for Linear Operators. Classics of Mathematics, Springer, Berlin, 620 pp.

[148] Kemeny, J.G., Snell, J.L. (1960). Finite Markov Chains. D Van Nostrand, New York, $210 \mathrm{pp}$.

[149] Kevorkian, J., Cole, J.D. (1981, 1985) Perturbation Methods in Applied Mathematics. Applied Mathematical Sciences, 34, Springer, New York, 560 pp.

[150] Kevorkian, J., Cole, J.D. (1996, 2011). Multiple Scale and Singular Perturbation Methods. Applied Mathematical Sciences, 114, Springer, New York, 634 pp.

[151] Khasminskii, R.Z., Yin, G., Zhang, Q. (1996a). Singularly perturbed Markov chains: quasi-stationary distribution and asymptotic expansion. In: Proceedings of Dynamic Systems and Applications, Vol. 2, Atlanta, GA, 1995. Dynamic, Atlanta, GA, 301308.

[152] Khasminskii, R.Z., Yin, G., Zhang, Q. (1996b). Asymptotic expansions of singularly perturbed systems involving rapidly fluctuating Markov chains. SIAM J. Appl. Math., 56, no. 1, 277-293.

[153] Kim, D.S., Smith, R.L. (1995). An exact aggregation/disaggregation algorithm for large scale Markov chains. Naval Res. Logist., 42, no. 7, 1115-1128.

[154] Kokotović, P.V., Phillips, R.G., Javid, S.H. (1980). Singular perturbation modeling of Markov processes. In: Bensoussan, A., Lions, J.L. (Eds). Analysis and optimization of systems: Proceedings of the Fourth International Conference on Analysis and Optimization. Lecture Notes in Control and Information Science, 28, Springer, Berlin, 3-15.

[155] Konstantinov, M. Gu, D.W., Mehrmann, V., Petkov, P. (2003). Perturbation Theory for Matrix Equations. Studies in Computational Mathematics, 9, North-Holland, Amsterdam, xii+429 pp.

[156] Konstantinov, M.M., Petkov, P.H. (2008). Perturbation methods in linear algebra and control. Appl. Comput. Math., 7, no. 2, 141-161.

[157] Kontoyiannis, I., Meyn, S.P. (2003). Spectral theory and limit theorems for geometrically ergodic Markov processes. Ann. Appl. Probab., 13, no. 1, 304-362. 
[158] Korolyuk, D.V., Silvestrov D.S. (1983). Entry times into asymptotically receding domains for ergodic Markov chains. Teor. Veroyatn. Primen., 28, 410-420 (English translation in Theory Probab. Appl., 28, 432-442).

[159] Korolyuk, D.V., Silvestrov D.S. (1984). Entry times into asymptotically receding regions for processes with semi-Markov switchings. Teor. Veroyatn. Primen., 29, 539-544 (English translation in Theory Probab. Appl., 29, 558-563).

[160] Korolyuk, V.S. (1969). On asymptotical estimate for time of a semi-Markov process being in the set of states. Ukr. Mat. Zh., 21, 842-845 (English translation in Ukr. Math. J., 21, 705-710).

[161] Korolyuk, V.S. (1989). Stochastic Models of Systems. Naukova Dumka, Kiev, 208 pp.

[162] Korolyuk, V.S., Brodi, S.M., Turbin, A.F. (1974). Semi-Markov processes and their application. Probability Theory. Mathematical Statistics. Theoretical Cybernetics, Vol. 11, VINTI, Moscow, 1974, 47-97.

[163] Korolyuk, V.S., Korolyuk, V.V. (1999). Stochastic Models of Systems. Mathematics and its Applications, 469, Kluwer, Dordrecht, xii+185 pp.

[164] Korolyuk, V.S., Limnios, N. (1998). Diffusion approximation of integral functionals in merging and averaging scheme. Teor. Imovirn. Mat. Stat., 59, 99-105 (English translation in Theory Probab. Math. Statist., 59, 101-107).

[165] Korolyuk, V.S., Limnios, N. (1999). Diffusion approximation for integral functionals in the double merging and averaging scheme. Teor. İmovirn. Mat. Stat., 60, 77-84 (English translation in Theory Probab. Math. Statist., 60, 87-94).

[166] Korolyuk, V.S., Limnios, N. (2000). Evolutionary systems in an asymptotic split phase space. In: Limnios, N., Nikulin, M. (Eds). Recent Advances in Reliability Theory: Methodology, Practice and Inference. Birkhäuser, Boston, 145-161.

[167] Korolyuk, V.S., Limnios, N. (2002). Markov additive processes in a phase merging scheme. In: Korolyuk, V., Prokhorov, Yu., Khokhlov, V., Klesov, O. (Eds). Proceedings of the Conference Dedicated to the 90th Anniversary of Boris Vladimirovich Gnedenko, Kiev, 2002. Theory Stoch. Process., 8, no. 3-4, 213-225.

[168] Korolyuk, V.S., Limnios, N. (2004a). Average and diffusion approximation of stochastic evolutionary systems in an asymptotic split state space. Ann. Appl. Probab., 14, 489-516

[169] Korolyuk, V.S., Limnios, N. (2004b). Diffusion approximation of evolutionary systems with equilibrium in asymptotic split phase space. Teor. İmovirn. Mat. Stat., 70, 63-73 (English translation in Theory Probab. Math. Statist., 70, 71-82).

[170] Koroliuk, V.S., Limnios, N. (2005). Stochastic Systems in Merging Phase Space. World Scientific, Singapore, xv+331 pp.

[171] Korolyuk, V.S., Mamonova, G.V. (2003) Stationary phase consolidation of the superposition of two renewal processes. Dopov. Nats. Akad. Nauk Ukr., Mat. Prirodozn. Tekh. Nauki, no. 5, 21-25 
[172] Korolyuk, V.S., Penev, I.P., Turbin, A.F. (1972). The asymptotic behavior of the distribution of the absorption time of a Markov chain. Kibernetika, no. 2, 20-22.

[173] Korolyuk, V.S., Penev, I.P., Turbin, A.F. (1981). Asymptotic expansion for the distribution of the absorption time of a weakly inhomogeneous Markov chain. In: Korolyuk, V.S. (Ed). Analytic Methods of Investigation in Probability Theory. Akad. Nauk Ukr. SSR, Inst. Mat., Kiev, 97-105.

[174] Korolyuk, V., Swishchuk, A. (1992). Semi-Markov Random Evolutions. Naukova Dumka, Kiev, 254 pp (English revised edition: Semi-Markov Random Evolutions. Mathematics and its Applications, 308, Kluwer, Dordrecht, 1995, x+310 pp.).

[175] Korolyuk, V.V., Tadzhiev, A. (1986). Asymptotic behavior of Markov evolutions prior to the time of absorption. Ukr. Mat. Zh., 38, 248-251 (English translation in Ukr. Math. J., 38, 219-222).

[176] Korolyuk, V.S., Turbin, A.F. (1970). On the asymptotic behaviour of the occupation time of a semi-Markov process in a reducible subset of states. Teor. Veroyatn. Mat. Stat., 2, 133-143 (English translation in Theory Probab. Math. Statist., 2, 133-143).

[177] Korolyuk, V.S., Turbin, A.F. (1972). A certain method of proving limit theorems for certain functionals of semi-Markov processes. Ukr. Mat. Zh., 24, 234-240.

[178] Korolyuk, V.S., Turbin, A.F. (1976). Semi-Markov Processes and its Applications. Naukova Dumka, Kiev, 184 pp.

[179] Korolyuk, V.S., Turbin, A.F. (1978). Mathematical Foundations of the State Lumping of Large Systems. Naukova Dumka, Kiev, 218 pp. (English edition: Mathematics and its Applications, 264, Kluwer, Dordrecht, 1993, x+278 pp.).

[180] Korolyuk, V.S., Turbin, A.F., Tomusjak, A.A. (1979). Sojourn time of a semiMarkov process in a decomposing set of states. In: Analytical Methods of Probability Theory, 152, Naukova Dumka, Kiev, 69-79

[181] Koury, J.R., McAllister, D.F., Stewart, W.J. (1984). Iterative methods for computing stationary distributions of nearly completely decomposable Mrkov chains. SIAM J. Alg. Disc. Melh., 5, pp. 164-186.

[182] Kovalenko, I.N. (1973). An algorithm of asymptotic analysis of a sojourn time of Markov chain in a set of states. Dokl. Acad. Nauk Ukr. SSR, Ser. A, no. 6, 422-426.

[183] Kovalenko, I.N. (1975). Studies in the Reliability Analysis of Complex Systems. Naukova Dumka, Kiev, 210 pp.

[184] Kovalenko, I.N., Kuznetsov, N.Yu., Pegg, P.A. (1997). Mathematical Theory of Reliability of Time Dependent Systems with Practical Applications. Wiley Series in Probability and Statistics, Wiley, New York, $316 \mathrm{pp}$.

[185] Langville, A.N., Meyer, C.D. (2006). Updating Markov chains with an eye on Google's PageRank. SIAM J. Matrix Anal. Appl., 27, no. 4, 968-987.

[186] Lasserre, J.B. (1994). A formula for singular perturbations of Markov chains. J. Appl. Probab., 31, 829-833.

[187] Latouche, G. (1988). Perturbation analysis of a phase-type queue with weakly correlated arrivals. Adv. Appl. Probab., 20, 896-912. 
[188] Latouche, G. (1991). First passage times in nearly decomposable Markov chains. In: Stewart, W.J. (Ed). Numerical Solution of Markov Chains. Probability: Pure and Applied, 8, Marcel Dekker, New York, 401-411.

[189] Latouche, G., Louchard, G. (1978). Return times in nearly decomposable stochastic processes. J. Appl. Probab., 15, 251-267.

[190] Latouche, G., Ramaswami, V. (1987, 1999) Introduction to Matrix Analytic Methods in Stochastic Modeling. ASA-SIAM Series on Statistics and Applied Probability, SIAM, Philadelphia, PA and ASA, Alexandria, VA, 348 pp.

[191] Leadbetter, M.R. (1963). On series expansion for the renewal moments. Biometrika, 50, no. 1-2, 75-80.

[192] Li, R.C., Stewart, G.W. (2000). A new relative perturbation theorem for singular subspaces. Linear Algebra Appl., 313, no. 1-3, 41-51.

[193] Li, X., Yin, G., Yin, K., Zhang, Q. (1999). A numerical study of singularly perturbed Markov chains: quasi-equilibrium distributions and scaled occupation measures, $D y$ nam. Contin. Discrete Impuls. Systems, 5, no. 1-4, 295304.

[194] Louchard, G., Latouche, G. (1982). Random times in nearly-completely decomposable, transient Markov chains. Cahiers Centre Études Rech. Opér., 24, no. 2-4, $321-352$.

[195] Louchard, G., Latouche, G. (1990). Geometric bounds on iterative approximations for nearly completely decomposable Markov chains. J. Appl. Probab., 27, no. 3, $521-529$.

[196] Marek, I., Mayer, P. (1998). Convergence analysis of an iterative aggregation/disaggregation method for computing stationary probability vectors of stochastic matrices. Numer. Linear Algebra Appl., 5, no. 4, 253-274.

[197] Mattingly, R.B., Meyer, C.D. (1991). Computing the stationary distribution vector of an irreducible Markov chain on a shared-memory multiprocessor. In: Stewart, W.J. (Ed). Numerical solution of Markov chains. Probability: Pure and Applied, 8. Marcel Dekker, Inc., New York, 491-510.

[198] McAllister, D.F., Stewart, G.W., Stewart, W.J. (1984). On a Rayleigh-Ritz refinement technique for nearly uncoupled stochastic matrices. Linear Algebra Appl., 60, $1-25$.

[199] Meshalkin, L.D. (1958). Limit theorems for Markov chains with a finite number of states. Teor. Veroyatn. Primen., 3, 361-385 (English translation in Theory Probab. Appl., 3, 335-357).

[200] Meyer, C.D. (1989). Stochastic complementation, uncoupling Markov chains, and the theory of nearly reducible systems. SIAM Rev., 31, no. 2, 240272.

[201] Meyer, C.D. (1994). Sensitivity of the stationary distribution of a Markov chain. SIAM J. Matrix Anal. Appl., 15, no. 3, 71-728.

[202] Meyer, C.D. (2000). Matrix Analysis and Applied Linear Algebra. SIAM, Philadelphia, PA, xii +718 pp. 
[203] Meyer, C.D. (2015). Continuity of the Perron root. Linear Multilinear Algebra, 63, no. $7,1332-1336$.

[204] Meyer, C.D., Jr. (1980). The condition of a finite Markov chain and perturbation bounds for the limiting probabilities. SIAM J. Algebr. Discr. Methods, 1, no. 3, 273-283.

[205] Meyer, C.D., Stewart, G.W. (1988). Derivatives and perturbations of eigenvectors. SIAM J. Numer. Anal., 25, no. 3, 679-691.

[206] Meyn, S.P., Tweedie, R.L. (1993, 2009). Markov Chains and Stochastic Stability. Communications and Control Engineering Series, Springer, London, 548 pp. and Cambridge University Press, 624 pp.

[207] Nagaev, S.V. (1957). Some limit theorems for stationary Markov chains. Teor. Veroyatn. Primen., 2, 389-416 (English translation in Theory Probab. Appl., 2, 378-406).

[208] Nagaev, S.V. (1961). A refinement of limit theorems for homogeneous Markov chains. Teor. Veroyatn. Primen., 6, 67-86 (English translation in Theory Probab. Appl., 6, $62-81)$.

[209] Ni, Y. (2010a). Perturbed renewal equations with multivariate nonpolynomial perturbations. In: Frenkel I., Gertsbakh I., Khvatskin L., Laslo Z., Lisnianski A. (Eds). Proceedings of the International Symposium on Stochastic Models in Reliability Engineering, Life Science and Operations Management. Beer Sheva, Israel, 754-763.

[210] Ni, Y. (2010b). Analytical and numerical studies of perturbed renewal equations with multivariate non-polynomial perturbations. J. Appl. Quant. Meth., 5, no. 3, 498-515.

[211] Ni, Y. (2011). Nonlinearly Perturbed Renewal Equations: Asymptotic Results and Applications. Doctoral dissertation, 106, Mälardalen University, Västerås.

[212] Ni, Y. (2012). Nonlinearly perturbed renewal equations: the non-polynomial case. Teor. Imovirn. Mat. Stat., 84, 111-122 (Also in Theory Probab. Math. Statist., 84, (2012), 117-129).

[213] Ni, Y. (2014). Exponential asymptotical expansions for ruin probability in a classical risk process with non-polynomial perturbations. In: Silvestrov, D., Martin-Löf, A. (Eds). Modern Problems in Insurance Mathematics. Chapter 6, EAA series, Springer, Cham, 67-91.

[214] Ni, Y., Silvestrov, D., Malyarenko, A. (2008). Exponential asymptotics for nonlinearly perturbed renewal equation with non-polynomial perturbations. J. Numer. Appl. Math., 1(96), 173-197.

[215] Paige, C.C., Styan, G.P.H., Wachter, P.G. (1975). Computation of the stationary distribution of a Markov chain. J. Statist. Comput. Simul., 4, 173-186.

[216] Pervozvanskiı̆, A.A., Gaitsgori, V.G. (1988). Theory of Suboptimal Decisions Decomposition and Aggregation. Mathematics and Its Applications (Soviet Series), 12, Kluwer, Dordrecht, xviii+384 pp. 
[217] Pervozvanskiŭ, A.A., Smirnov, I.N. (1974). An estimate of the steady state of a complex system with slowly varying constraints. Kibernetika, no. 4, 45-51 (English translation in Cybernetics, 10, (1976), no. 4, 603-611).

[218] Petersson, M. (2013a). Quasi-stationary distributions for perturbed discrete time regenerative processes. Teor. Imovirn. Mat. Stat., 89, 140-155 (Also in Theor. Probab. Math. Statist., 89, (2014), 153-168).

[219] Petersson, M. (2013b). Asymptotic Expansions for Perturbed Discrete Time Renewal Equations. Licentiate Thesis. Stockholm University.

[220] Petersson, M. (2014). Asymptotics of ruin probabilities for perturbed discrete time risk processes. In: Silvestrov, D., Martin-Löf, A. (Eds). Modern Problems in Insurance Mathematics, Chapter 7, EAA series, Springer, Cham, 93-110.

[221] Phillips, R.G., Kokotović, P.V. (1981). A singular perturbation approach to modeling and control of Markov chains. IEEE Trans. Auto, Contr., 26, 1087-1094.

[222] Plotkin, J.D., Turbin, A.F. (1971). Inversion of linear operators that are perturbed on the spectrum. Ukr.. Mat. Zh., 23, 168-176.

[223] Plotkin, J.D., Turbin, A.F. (1975). Inversion of normally solvable linear operators that are perturbed on the spectrum. Ukr. Mat. Zh., 27, no. 4, 477-486,

[224] Poliščuk, L.I., Turbin, A.F. (1973). Asymptotic expansions for certain characteristics of semi-Markov processes. Teor. Veroyatn. Mat. Stat., 8 122-127 (English translation in Theory Probab. Math. Statist., 8, 121-126).

[225] Pollett, P.K., Stewart, D.E. (1994). An efficient procedure for computing quasistationary distributions of Markov chains with sparse transition structure. Adv. Appl. Probab., 26, 68-79.

[226] Quadrat, J.P. (1983). Optimal control of perturbed Markov chains: the multitime scale case. In: Ardema, M.D. (Ed). Singular Perturbations in Systems and Control, CISM Courses and Lectures, 280, Springer, Vienna, 215-239.

[227] Rohlichek, J.R., (1987). Aggregation and Time Scale Analysis of Perturbed Markov Systems. Ph.D. Thesis, Massachusetts Inst. Tech., Cambridge, MA.

[228] Rohlicek, J.R., Willsky, A.S. (1988a). Multiple time scale decomposition of discrete time Markov chains. Systems Control Lett., 11, no. 4, 309-314.

[229] Rohlicek, J.R., Willsky, A.S. (1988b). The reduction of perturbed Markov generators: an algorithm exposing the role of transient states. J. Assoc. Comput. Mach., 35, no. 3, 675696 .

[230] Romanovskiı̌, V.I. (1949). Discrete Markov Chains. Gostehizdat, MoscowLeningrad, 436 pp.

[231] Samollenko, I.V. (2006a). Asymptotic expansion of Markov random evolution. Ukr. Mat. Visn., 3, no. 3, 394-407 (English translation in Ukr. Math. Bull., 3, no. 3, 381-394).

[232] Samollenko, İ.V. (2006b). Asymptotic expansion of semi-Markov random evolution. Ukr. Mat. Zh., 58, 1234-1248 (English translation in Ukr. Math. J., 58, 1396-1414). 
[233] Schweitzer, P.J. (1968). Perturbation theory and finite Markov chains. J. Appl. Probab., 5, 401-413.

[234] Schweitzer, P.J. (1984). Aggregation methods for large Markov chains. In. Giuseppe Iazeolla, G., Courtois, P.J., Hordijk, A. (Eds). Mathematical Computer Performance and Reliability, North-Holland, Amsterdam, 275-286.

[235] Schweitzer, P.J. (1986). Posterior bounds on the equilibrium distribution of a finite Markov chain. Comm. Statist. Stoch. Models, 2, no. 3, 323-338.

[236] Schweitzer, P.J. (1987). Dual bounds on the equilibrium distribution of a finite Markov chain. J. Math. Anal. Appl., 126, no. 2, 478-482.

[237] Schweitzer, P.J. (1991). A survey of aggregation-disaggregation in large Markov chains. In: Stewart, W.J. (Ed). Numerical solution of Markov chains. Probability: Pure and Applied, 8. Marcel Dekker, Inc., New York, 63-88.

[238] Schweitzer, P.J., Kindle, K.W. (1986). Iterative aggregation for solving undiscounted semi-Markovian reward processes. Comm. Statist. Stoch. Models, 2, no. 1, 1-41.

[239] Schweitzer, P.J., Puterman, M.L., Kindle, K.W. (1985). Iterative aggregationdisaggregation procedures for discounted semi-Markov reward processes. Oper. Res., 33, no. 3, 589-605.

[240] Schweitzer, P., Stewart, G.W. (1993). The Laurent expansion of pencils that are singular at the origin. Linear Algebra Appl., 183, 237-254.

[241] Seneta, E. (1967). Finite approximations to infinite non-negative matrices. Proc. Cambridge Philos. Soc., 63, 983-992.

[242] Seneta, E. (1968a). Finite approximations to infinite non-negative matrices. II. Refinements and applications. Proc. Cambridge Philos. Soc., 64, 465-470.

[243] Seneta, E. (1968b). The principle of truncations in applied probability. Comment. Math. Univ. Carolinae, 9, 237-242.

[244] Seneta, E. (1973). Non-negative Matrices. An Introduction to Theory and Applications. Wiley, New York, 256 pp.

[245] Seneta, E. (1984). Iterative aggregation: convergence rate. Econom. Lett., 14, no. $4,357-361$.

[246] Seneta, E. (1988a). Sensitivity to perturbation of the stationary distribution: some refinements. Linear Algebra Appl., 108, 121-126.

[247] Seneta, E. (1988b). Perturbation of the stationary distribution measured by ergodicity coefficients. Adv. Appl. Probab., 20, 228-230.

[248] Seneta, E. (1991). Sensitivity analysis, ergodicity coefficients, and rank-one updates for finite Markov chains. In: Stewart, W.J. (Ed). Numerical Solution of Markov Chains. Probability: Pure and Applied, 8, Marcel Dekker, New York, 121-129.

[249] Seneta, E. (1993). Sensitivity of finite Markov chains under perturbation. Statist. Probab. Lett., 17, no. 2, 163-168. 
[250] Seneta, E. (2006). Non-negative matrices and Markov chains. Revised reprint of the second (1981) edition, Springer Series in Statistics. Springer, New York, 2006. xvi+287 pp.

[251] Sheskin, T.J. (1985). A Markov chain partitioning algorithm for computing steady state probabilities. Oper. Res., 33, 228-235.

[252] Shurenkov, V.M. (1980a). Transition phenomena of the renewal theory in asymptotical problems of theory of random processes 1. Mat. Sbornik, 112, 115-132 (English translation in Math. USSR: Sbornik, 40, no. 1, 107-123).

[253] Shurenkov, V.M. (1980b). Transition phenomena of the renewal theory in asymptotical problems of theory of random processes 2. Mat. Sbornik, 112, 226-241 (English translation in Math. USSR: Sbornik, 40, no. 2, 211-225).

[254] Silvestrov, D.S. (1969). Limit theorems for a non-recurrent walk connected with a Markov chain. Ukr. Mat. Zh., 21, 790-804 (English translation in Ukr. Math. J., 21, 657-669).

[255] Silvestrov, D.S. (1970). Limit theorems for semi-Markov processes and their applications. 1, 2. Teor. Veroyatn. Mat. Stat., 3, 155-172, 173-194 (English translation in Theory Probab. Math. Statist., 3, 159-176, 177-198).

[256] Silvestrov, D.S. (1971a) Limit theorems for semi-Markov summation schemes. 1. Teor. Veroyatn. Mat. Stat., 4, 153-170 (English translation in Theory Probab. Math. Statist., 4, 141-157).

[257] Silvestrov, D. S. (1971b). Uniform estimates of the rate of convergencxe for sums of random variables defined on a finite homogeneous Markov chain with absorption. Theor. Veroyatn. Mat. Stat., 5, 116-127 (English translation in Theory Probab. Math. Statist., 5, 123-135).

[258] Silvestrov, D.S. (1972a). Limit distributions for sums of random variables that are defined on a countable Markov chain with absorption. Dokl. Acad. Nauk Ukr. SSR, Ser. A, no. 4, 337-340.

[259] Silvestrov, D.S. (1972b). Estimation of the rate of convergence for sums of random variables that are defined on a countable Markov chain with absorption. Dokl. Acad. Nauk Ukr. SSR, Ser. A, no. 5, 436-438.

[260] Silvestrov, D.S. (1974). Limit Theorems for Composite Random Functions. Vysshaya Shkola and Izdatel'stvo Kievskogo Universiteta, Kiev, 318 pp.

[261] Silvestrov, D.S. (1976). A generalization of the renewal theorem. Dokl. Akad. Nauk Ukr. SSR, Ser. A, no. 11, 978-982.

[262] Silvestrov, D.S. (1978). The renewal theorem in a series scheme. 1. Teor. Veroyatn. Mat. Stat., 18, 144-161 (English translation in Theory Probab. Math. Statist., 18, 155-172).

[263] Silvestrov, D.S. (1979a). The renewal theorem in a series scheme 2. Teor. Veroyatn. Mat. Stat., 20, 97-116 (English translation in Theory Probab. Math. Statist., 20, 113-130). 
[264] Silvestrov, D.S. (1979b). A remark on limit distributions for times of attainment for asymptotically recurrent Markov chains. Theory Stoch. Process., 7, 106-109.

[265] Silvestrov, D.S. (1980a). Semi-Markov Processes with a Discrete State Space. Library for the Engineer in Reliability, Sovetskoe Radio, Moscow, 272 pp.

[266] Silvestrov, D.S. (1980b). Mean hitting times for semi-Markov processes, and queueing networks. Elektron. Infor. Kybern., 16, 399-415.

[267] Silvestrov, D.S. (1981). Theorems of large deviations type for entry times of a sequence with mixing. Teor. Veroyatn. Mat. Stat., 24, 129-135 (English translation in Theory Probab. Math. Statist., 24, 145-151).

[268] Silvestrov, D.S. (1995). Exponential asymptotic for perturbed renewal equations. Teor. Imovirn. Mat. Stat., 52, 143-153 (English translation in Theory Probab. Math. Statist., 52, 153-162).

[269] Silvestrov, D.S. (1996). Recurrence relations for generalised hitting times for semiMarkov processes. Ann. Appl. Probab., 6, 617-649.

[270] Silvestrov, D.S. (2000). Nonlinearly perturbed Markov chains and large deviations for lifetime functionals. In: Limnios, N., Nikulin, M. (Eds). Recent Advances in Reliability Theory: Methodology, Practice and Inference. Birkhäuser, Boston, 135144.

[271] Silvestrov, D.S. (2007). Asymptotic expansions for quasi-stationary distributions of nonlinearly perturbed semi-Markov processes. Theory Stoch. Process., 13, no. 1-2, 267-271.

[272] Silvestrov D.S. (2010). Nonlinearly Perturbed Stochastic Processes and Systems. In: Rykov, V., Balakrishnan, N., Nikulin, M. (Eds). Mathematical and Statistical Models and Methods in Reliability. Birkhäuser, Chapter 2, 19-38.

[273] Silvestrov D.S. (2014). Improved asymptotics for ruin probabilities. In: Silvestrov, D., Martin-Löf, A. (Eds). Modern Problems in Insurance Mathematics, Chapter 5, EAA series, Springer, Cham, 93-110.

[274] Silvestrov D.S. (2014). American-Type Options. Stochastic Approximation Methods, Volume 1. De Gruyter Studies in Mathematics, 56, Walter de Gruyter, Berlin, $\mathrm{x}+509$ pp.

[275] Silvestrov D.S. (2015). American-Type Options. Stochastic Approximation Methods, Volume 2. De Gruyter Studies in Mathematics, 57, Walter de Gruyter, Berlin, $\mathrm{xi}+558 \mathrm{pp}$.

[276] Silvestrov, D.S., Abadov, Z.A. (1984). Asymptotic behaviour for exponential moments of sums of random variables defined on exponentially ergodic Markov chains. Dokl. Acad. Nauk Ukr. SSR, Ser. A, no. 4, 23-25.

[277] Silvestrov, D.S., Abadov, Z.A. (1991). Uniform asymptotic expansions for exponential moments of sums of random variables defined on a Markov chain and distributions of passage times. 1. Teor. Veroyatn. Mat. Stat., 45, 108-127 (English translation in Theory Probab. Math. Statist., 45, 105-120). 
[278] Silvestrov, D.S., Abadov, Z.A. (1993). Uniform representations of exponential moments of sums of random variables defined on a Markov chain, and of distributions of passage times. 2. Teor. Veroyatn. Mat. Stat., 48, 175-183 (English translation in Theory Probab. Math. Statist., 48, 125-130).

[279] Silvestrov, D.S., Drozdenko, M.O. (2005). Necessary and sufficient conditions for the weak convergence of the first-rare-event times for semi-Markov processes. Dopov. Nac. Akad. Nauk Ukr., Mat. Prirodozn. Tekh. Nauki, no. 11, 25-28.

[280] Silvestrov, D.S., Drozdenko, M.O. (2006). Necessary and sufficient conditions for weak convergence of first-rare-event times for semi-Markov processes. Theory Stoch. Process., 12(28), no. 3-4, Part I: 151-186, Part II: 187-202.

[281] Silvestrov, D., Manca, R., Silvestrova, E. (2014). Computational algorithms for moments of accumulated Markov and semi-Markov rewards. Comm. Statist. Theory, Meth., 43, no. 7, 1453-1469.

[282] Silvestrov, D.S., Petersson, M. (2013). Exponential expansions for perturbed discrete time renewal equations. In: Karagrigoriou, A., Lisnianski, A., Kleyner, A., Frenkel, I. (Eds). Applied Reliability Engineering and Risk Analysis. Probabilistic Models and Statistical Inference. Chapter 23, Wiley, 349-362.

[283] Silvestrov, D., Silvestrov, S. (2015). Asymptotic expansions for stationary distributions of perturbed semi-Markov processes. Research Report 2015-9, Department of Mathematics, Stockholm University, 75 pp.

[284] Silvestrov, D.S., Velikii, Y.A. (1988). Necessary and sufficient conditions for convergence of attainment times. In: Zolotarev, V.M., Kalashnikov, V.V. (Eds). Stability Problems for Stochastic Models. Trudy Seminara, VNIISI, Moscow, 129-137 (English translation in J. Soviet. Math., 57, 3317-3324).

[285] Simon, H.A., Ando, A. (1961). Aggregation of variables in dynamic systems. Econometrica, 29, 111-138.

[286] Sirl, D., Zhang, H., Pollett, P. (2007). Computable bounds for the decay parameter of a birth-death process. J. Appl. Probab., 44, no. 2, 476-491.

[287] Stewart, G.W. (1969). On the continuity of the generalized inverse. SIAM J. Appl. Math., 17, 33-45.

[288] Stewart, G.W. (1973). Error and perturbation bounds for subspaces associated with certain eigenvalue problems. SIAM Rev., 15, 727-764.

[289] Stewart, G.W. (1979). Perturbation bounds for the definite generalized eigenvalue problem. Linear Algebra Appl., 23, 69-85.

[290] Stewart, G.W. (1983). Computable error bounds for aggregated Markov chains. J. Assoc. Comput. Mach., 30, no. 2, 271-285.

[291] Stewart, G.W. (1984a). On the structure of nearly uncoupled Markov chains. In: Iazeolla, G, Courtois, P.J., Hordijk, A. (Eds). Mathematical Computer Performance and Reliability. North-Holland, Amsterdam, 287-302.

[292] Stewart, G.W. (1984b). A second order perturbation expansion for small singular values. Linear Algebra Appl., 56, 231-235. 
[293] Stewart, G.W. (1990). Stochastic perturbation theory. SIAM Rev., 32, no. 4, 579610.

[294] Stewart, G.W. (1991). On the sensitivity of nearly uncoupled Markov chains. In: Stewart, W.J. (Ed). Numerical Solution of Markov Chains. Probability: Pure and Applied, 8, Marcel Dekker, New York, 105-119.

[295] Stewart, G.W. (1993a). Gaussian elimination, perturbation theory, and Markov chains. In: Meyer, C.D., Plemmons, R.J. (Eds). Linear Algebra, Markov Chains, and Queueing Models. IMA Volumes in Mathematics and its Applications, 48, Springer, New York, 59-69.

[296] Stewart, G.W. (1993b). On the perturbation of Markov chains with nearly transient states. Numer. Math., 65, no. 1, 135-141.

[297] Stewart, G.W. (1998). Matrix Algorithms. Vol. I. Basic Decompositions. SIAM, Philadelphia, PA, xx+458 pp.

[298] Stewart, G.W. (2001). Matrix Algorithms. Vol. II. Eigensystems. SIAM, Philadelphia, PA, $\mathrm{xx}+469 \mathrm{pp}$.

[299] Stewart, G.W. (2003). On the powers of a matrix with perturbations. Numer. Math., 96, no. 2, 363376 .

[300] Stewart, G.W., Stewart, W.J., McAllister, D.F. (1994). A two-stage iteration for solving nearly completely decomposable Markov chains. In: Golub, G., Greenbaum, A., Luskin, M. (Eds). Recent Advances in Iterative Methods. IMA Volumes in Mathematics and its Applications, 60, Springer, New York, 201-216.

[301] Stewart, G.W., Sun, J.G. (1990). Matrix Perturbation Theory. Computer Science and Scientific Computing. Academic Press, Boston, xvi+365 pp.

[302] Stewart, G.W., Zhang, G. (1991). On a direct method for the solution of nearly uncoupled Markov chains. Numer. Math., 59, no. 1, 1-11.

[303] Stewart, W.J. (1994). Introduction to the Numerical Solution of Markov Chains. Princeton University Press, Princeton, NJ, xx+539 pp.

[304] Sumita, U., Reiders, M. (1988). A new algorithm for computing the ergodic probability vector for large Markov chains: Replacement process approach. Probab. Eng. Infor. Sci., 4, 89-116.

[305] Torokhti, A., Howlett, P., Pearce, C. (2003). Method of best successive approximations for nonlinear operators. J. Comput. Anal. Appl., 5, no. 3, 299-312.

[306] Turbin, A.F. (1971). On asymptotic behavior of time of a semi-Markov process being in a reducible set of states. Linear case. Teor. Veroyatn. Mat. Stat., 4, 179194 (English translation in Theory Probab. Math. Statist., 4, 167-182).

[307] Turbin, A.F. (1972). An application of the theory of perturbations of linear operators to the solution of certain problems that are connected with Markov chains and semiMarkov processes. Teor. Veroyatn. Mat. Stat., 6, 118-128 (English translation in Theory Probab. Math. Statist., 6, 119-130).

[308] Vantilborgh, H. (1985). Aggregation with an error of $\mathrm{O}\left(\epsilon^{2}\right)$. J. Assoc. Comput. Mach., 32, no. 1, 162190. 
[309] Verhulst, F. ( 2005). Methods and Applications of Singular Perturbations: Boundary Layers and Multiple Timescale Dynamics. Texts in Applied Mathematics, 50, Springer, New York, xvi+324 pp.

[310] Vishik, M.I., Lyusternik, L.A. (1960). The solution of some perturbation problems in the case of matrices and self-adjoint and non-self-adjoint differential equations. Uspehi Mat. Nauk, 15, 3-80.

[311] Wentzell, A.D. (1996). Asymptotic expansions in limit theorems for stochastic processes. I. Probab. Theory Related Fields, 106, no. 3, 331-350.

[312] Wentzell, A.D. (1999). Asymptotic expansions in limit theorems for stochastic processes. II. Probab. Theory Related Fields, 113, no. 2, 255-271.

[313] Wentzell, A.D., Freidlin, M.I. (1979). Fluctuations in Dynamical Systems Subject to Small Random Perturbations. Probability Theory and Mathematical Statistics, Nauka, Moscow, 424 pp. (English edition: Random Perturbations of Dynamical Systems. Fundamental Principles of Mathematical Sciences, 260, Springer, New York (1998, 2012), xxviii+458 pp).

[314] Wilkinson, J.H. (1961). Error analysis of direct method of matrix inversion. J. Ass. Comp. Mach., 8, 281-330.

[315] Yin, G.G., Zhang, Q. (1998). Continuous-time Markov Chains and Applications. A Singular Perturbation Approach. Applications of Mathematics, 37, Springer, New York, ivx+349 pp.

[316] Yin, G., Zhang, Q. (2003). Discrete-time singularly perturbed Markov chains. In: Yao, D.D., Zhang, H., Zhou, X.Y. (Eds). Stochastic Modeling and Optimization. Springer, New York, 1-42.

[317] Yin, G.G., Zhang, Q. (2005). Discrete-time Markov chains. Two-time-scale methods and applications. Stochastic Modelling and Applied Probability, Springer, New York, xix +348 pp.

[318] Yin, G.G., Zhang, Q. (2013). Continuous-time Markov chains and applications. A two-time-scale approach. Second edition. Stochastic Modelling and Applied Probability, 37, Springer, New York, xxii+427 pp.

[319] Yin, G., Zhang, Q., Badowski, G. (2003). Discrete-time singularly perturbed Markov chains: aggregation, occupation measures, and switching diffusion limit. Adv. Appl. Probab., 35, no. 2, 449-476.

[320] Yin, G., Zhang, Q., Yang, H., Yin, K. (2001). Discrete-time dynamic systems arising from singularly perturbed Markov chains. In: Proceedings of the Third World Congress of Nonlinear Analysts, Part 7, Catania, 2000. Nonlinear Anal., 47, no. 7, $4763-4774$.

[321] Zhang, Q., Yin, G. (2004). Exponential bounds for discrete-time singularly perturbed Markov chains. J. Math. Anal. Appl., 293, no. 2, 645-662. 